\title{
Hall Viscosity in Quantum Systems with Discrete Symmetry: Point Group and Lattice Anisotropy
}

\author{
Pranav Rao and Barry Bradlyn $\odot$ \\ Department of Physics and Institute for Condensed Matter Theory, \\ University of Illinois at Urbana-Champaign, Urbana, Illinois 61801-3080, USA
}

(Received 27 October 2019; accepted 28 February 2020; published 7 April 2020)

\begin{abstract}
Inspired by recent experiments on graphene, we examine the nondissipative viscoelastic response of anisotropic two-dimensional quantum systems. We pay particular attention to electron fluids with point group symmetries and those with discrete translational symmetry. We start by extending the Kubo formalism for viscosity to systems with internal degrees of freedom and discrete translational symmetry, highlighting the importance of properly considering the role of internal angular momentum. We analyze the Hall components of the viscoelastic response tensor in systems with discrete point group symmetry, focusing on the hydrodynamic implications of the resulting forces. We show that though there are generally six Hall viscosities, there are only three independent contributions to the viscous force density in the bulk. To compute these coefficients, we develop a framework to consistently write down the long-wavelength stress tensor and viscosity for multicomponent lattice systems. We apply our formalism to lattice and continuum models, including a lattice Chern insulator and anisotropic superfluid.
\end{abstract}

DOI: 10.1103/PhysRevX.10.021005

Subject Areas: Condensed Matter Physics

\section{INTRODUCTION}

One of the most peculiar and fascinating manifestations of topology in condensed matter physics is the appearance of nondissipative transport coefficients in insulating systems. The paradigmatic example is Hall conductivity, which is quantized in a two-dimensional insulator and proportional to a topological invariant- the Chern numbercharacterizing the many-body ground state [1-3]. Similarly, it has recently been noted that in two-dimensional insulators with broken time-reversal symmetry, there is a nondissipative viscosity [4-6]. In rotationally invariant phases, it has been shown that there is a unique Hall viscosity coefficient $\eta^{\mathrm{H}}$, which for a gapped phase is proportional to the particle density $\bar{n}$ and a quantized invariant of the ground state known as the shift $\mathcal{S}$ [6,7],

$$
\eta^{\mathrm{H}}=\frac{\hbar}{4} \bar{n} \mathcal{S} .
$$

The shift quantifies the number of additional magnetic monopoles needed to stabilize the ground state on a sphere [8]. In the quantum Hall regime, the Hall viscosity has been

\footnotetext{
* Corresponding author. bbradlyn@illinois.edu

Published by the American Physical Society under the terms of the Creative Commons Attribution 4.0 International license. Further distribution of this work must maintain attribution to the author(s) and the published article's title, journal citation, and DOI.
}

proposed as a numerical diagnostic for distinguishing between different competing topological orders [9]. When rotational symmetry is broken, there ceases to be a single Hall viscosity coefficient, and the relation between the viscosity and the shift is lost [10-13].

Outside of insulators, topological considerations can also lead to nondissipative transport coefficients in metallic systems due to the influence of Berry phase effects in transport. Pioneering works by Karplus and Luttinger [14], as well as Haldane [15], have shown how the Hall conductivity in metallic magnets receives a contribution due to the Berry curvature of the occupied states in a Fermi liquid. Recently, there has been a surge of interest in the nondissipative viscosity of metallic systems as well, driven in large part by the discovery of hydrodynamic flow in systems like graphene [16]. For electronic fluids with an approximately conserved momentum at long wavelengths, experiments have been proposed for extracting the Hall viscosity from flow through the width dependence of the Hall conductance in narrow channels [17,18], the flow profile near point contacts $[19,20]$, and the semiclassical distribution function [21]. Cutting-edge experiments in graphene under nonquantizing magnetic fields have started to validate these proposals [22]. Hall viscosity is also an area of active theoretical study, with work on graphene [23-25] and the consideration of viscous effects in a variety of other contexts [26-29] ongoing.

Despite this progress, the robustness and even the definability of the Hall viscosity in the absence of rotational and translational symmetry have not been systematically 
treated. For instance, the low-energy Dirac theory of graphene arises as a $\mathbf{k} \cdot \mathbf{p}$ expansion in a highly anisotropic band structure for a system with no translational symmetry. In spite of previous works examining the Hall viscosity in models with broken translational symmetry [30-33], the connection between microscopic, low-energy descriptions and long-wavelength hydrodynamics relevant to experiment has not been directly addressed. Furthermore, a comprehensive framework for treating momentum transport in systems with broken time-reversal symmetry and no external magnetic field (analogous to the formalism for the anomalous Hall conductance) is lacking.

In this work, we take steps to address these issues by developing a formalism for nondissipative viscosity in nonrotationally invariant systems, both in the continuum and with periodic potentials. With these tools, we deduce several conclusions about the viscosity of anisotropic quantum fluids. Our three main conceptual innovations are as follows: (1) a novel detailed analysis of the nondissipative viscosity tensor, revealing that although there are generally six viscosity coefficients, three are redundant in the bulk (we find a similar redundancy in the dissipative viscosity); (2) a relationship between band topology and Hall viscosity for free-fermion systems, showing how the six viscosity coefficients are expressible in terms of quadrupole moments of the Berry curvature of occupied bands and a correction due to the internal (pseudospin) angular momentum of bands; and (3) the first consistent framework for momentum transport and viscosity on a lattice or tight-binding system, derived only from conservation laws. In formulating these results, we also develop an extension of the Belinfante-Rosenfeld symmetrization procedure to anisotropic continuum and lattice systems, thus fixing the antisymmetric part of the stress tensor operator. Before continuing, we give some brief background and establish our notational conventions.

\section{A. Background and notation}

To begin, let us establish notation and review how viscosity arises in nonrelativistic quantum systems. Throughout this paper, we work in units where $\hbar=c=$ $e=1$. For a quantum system with a single-particle type in $d$ dimensions, we can introduce the stress tensor through the conservation law for momentum. We thus start with the momentum density operator $g_{\mu}(\mathbf{r})$. Here and throughout this work, Greek indices such as $\mu$ run over spatial directions $\mu=1, \ldots, d$, and unless otherwise noted, we take $d=2$. The stress tensor $\tau^{\mu}{ }_{\nu}(\mathbf{r})$ can then be defined through the conservation law for momentum density [34],

$$
\partial_{t} g_{\nu}(\mathbf{r})+\sum_{\mu} \partial_{\mu} \tau_{\nu}^{\mu}(\mathbf{r})=f_{\nu}^{\mathrm{ext}}(\mathbf{r}),
$$

where $\mathbf{f}^{\text {ext }}$ is the density of external forces acting on the particles. Throughout this work, we use boldface symbols to refer exclusively to two-dimensional vectors. We also introduce the shorthand $\partial_{\mu}$ to denote the partial derivative of a function with respect to a component of its (coordinate) vector argument,

$$
\partial_{\mu} f(\mathbf{x}) \equiv \frac{\partial f}{\partial x_{\mu}}
$$

and analogously in momentum space,

$$
\partial^{\mu} f(\mathbf{k}) \equiv \frac{\partial f}{\partial k^{\mu}}
$$

Furthermore, we do not use the Einstein summation convention in this work. Since expressions in lattice systems often involve repeated indices, we explicitly indicate all summations over indices as above. Because this is an unconventional choice, we remind the reader periodically that repeated indices are not summed over.

Equation (2) defines the stress tensor up to a divergenceless term, which must be fixed from other considerations. In rotationally invariant relativistic systems, it is always possible to choose a stress tensor that is symmetric in flat space (where we can avoid complications due to index raising or lowering) [35]. It was recently shown how to adapt this symmetrization procedure for rotationally invariant nonrelativistic two-component fermions as well [36]. One of the main results of our work is a generalization of this procedure to lattice and continuum systems that lack rotational symmetry.

Having defined the stress tensor, we are primarily interested in the response of the stress to an applied time-varying strain perturbation $\lambda_{\mu}{ }^{\nu} \equiv \partial_{\mu} v^{\nu}$. To make contact with classical hydrodynamics, we can interpret $v^{\nu}$ as a spatially dependent velocity field in a fluid. We expand the average of the stress tensor perturbatively in the velocity field to define

$$
\left\langle\tau^{\mu}{ }_{\nu}\right\rangle=\left\langle\tau_{\nu}^{\mu}\right\rangle_{0}-\sum_{\lambda \rho}\left[\kappa^{\mu}{ }_{\nu}{ }^{\lambda} \rho \int d t \partial_{\lambda} v^{\rho}+\eta^{\mu}{ }_{\nu}{ }^{\lambda}{ }_{\rho} \partial_{\lambda} v^{\rho}+\ldots\right] .
$$

Here, $\left\langle\tau^{\mu}{ }_{\nu}\right\rangle_{0}$ denotes the average stress in the absence of a strain perturbation. For a translation-invariant fluid in $d$ dimensions, we have that

$$
\left\langle\tau^{\mu}{ }_{\nu}\right\rangle_{0}=\mathcal{P} \delta_{\nu}^{\mu}
$$

where $\mathcal{P}$ is the hydrostatic pressure. The tensor ${\kappa^{\mu}}^{\mu}{ }_{\nu}{ }_{\rho}$ is the tensor of elastic moduli, and it gives the response of the stress tensor to static strains [hence the time integral in Eq. (5)]. Finally, the tensor $\eta^{\mu}{ }_{\nu}{ }_{\rho}^{\lambda}$ is the viscosity tensor, and it is the fundamental object of study for this work. In particular, we mostly focus on the Hall viscosity tensor 


$$
\left(\eta^{\mathrm{H}}\right)_{\nu \rho}^{\mu \lambda} \equiv \frac{1}{2}\left(\eta^{\mu}{ }_{\nu} \lambda_{\rho}-\eta^{\lambda}{ }_{\rho}^{\mu}{ }_{\nu}\right)
$$

which is the part of the viscosity tensor that does not contribute to power dissipation.

\section{B. Outline}

In the following sections, we examine the constraints that point group symmetry places on the momentum density, stress, and (Hall) viscosity tensors. The structure of the paper is as follows: First, in Sec. II, we show how to define the analogue of the Belinfante stress tensor in a translationinvariant, nonrelativistic anisotropic system. Then, in Sec. III, we extend this formalism to systems with only discrete translation symmetry. With the formalism developed, in Sec. IV, we investigate the constraints of point group symmetry on the viscosity tensor both phenomenologically and microscopically using the Kubo formalism. In Sec. V, we focus, in particular, on free-fermion systems, where we can relate the Hall viscosity to band topology. Finally, in Sec. VI, we apply these results to lattice and continuum models of interest.

\section{CONTINUUM SYSTEMS: STRAIN AND STRESS WITH ANISOTROPY}

To begin, let us consider a general Hamiltonian for an interacting, translation-invariant system of particles in two dimensions. We additionally assume that the particles have $N_{L}$ internal degrees of freedom. We can write the Hamiltonian for such a system as

$$
H=\sum_{i a} \mathbf{M}_{a} T^{a}\left(\mathbf{p}_{i}\right)+\frac{1}{2} \sum_{i \neq j, a} \mathbf{M}_{a} V^{a}\left(\mathbf{x}_{i}-\mathbf{x}_{j}\right),
$$

where $\mathbf{p}_{i}$ is the momentum operator for particle $i, \mathbf{x}_{i}$ is the position operator for particle $i$ : the two labels $i, j=$ $1, \ldots, N$ index the particles, and the matrices $\mathbf{M}_{a}$ form a basis for $N_{L} \times N_{L}$ Hermitian matrices. We have the canonical commutation relations

$$
\left[x_{i}^{\mu}, p_{\nu}^{j}\right]=i \delta_{\nu}^{\mu} \delta_{i}^{j} .
$$

To compute the viscosity for the ground state of such a system, we employ the Kubo formalism of Ref. [37]. To do so, we must first identify the momentum density operator $\mathbf{g}(\mathbf{r})$. If we ignore the internal degrees of freedom of the particles, then we have that all momentum must be carried by kinetic motion. Following the logic of Ref. [37], we write the momentum density as the density of kinetic momentum,

$$
\mathbf{g}_{\text {kin }}(\mathbf{r})=\frac{1}{2} \sum_{i}\left\{\mathbf{p}_{i}, \delta\left(\mathbf{r}-\mathbf{x}_{i}\right)\right\},
$$

where $\{\cdot, \cdot\}$ indicates the anticommutator. We can identify the first moment of the kinetic momentum density

$$
J_{\nu}^{\mu}=-\int d^{2} r r^{\mu} g_{\nu}(\mathbf{r})=-\frac{1}{2} \sum_{i}\left\{x_{i}^{\mu}, p_{\nu}^{i}\right\}
$$

with the generators of uniform spatial strains (i.e., positiondependent displacements). Furthermore, by taking the longwavelength limit of the Fourier transform of Eq. (2), we see that the time derivative of the strain generators gives the integrated (canonical) stress tensor

$$
T_{\nu}^{\mu}=\int d^{2} r \tau^{\mu}{ }_{\nu}(\mathbf{r}) \equiv-i\left[H, J_{\nu}^{\mu}\right] .
$$

Because Eq. (12) was derived from the kinetic momentum density, we refer to it as the kinetic stress.

However, when the internal degrees of freedom of the particles transform nontrivially under rotations, we must modify the momentum density to account for the density of internal linear momentum. To see this most directly, let us recall that the antisymmetric part of the strain generator should generate rotations. On the coordinates and the momenta, rotations act in the standard way as

$$
\begin{aligned}
& U(\theta) \mathbf{x}_{i} U^{\dagger}(\theta)=\sum_{\nu} R_{\nu}^{\mu} x_{i}^{\nu}, \\
& U(\theta) \pi^{i} U^{\dagger}(\theta)=\sum_{\nu} R_{\mu}^{\nu} \pi_{\nu}^{i},
\end{aligned}
$$

with

$$
R=\left(\begin{array}{cc}
\cos \theta & -\sin \theta \\
\sin \theta & \cos \theta
\end{array}\right)
$$

a rotation matrix. This action is generated by

$$
\left.i \frac{\partial U}{\partial \theta} U^{\dagger}\right|_{\theta=0}=L_{\mathrm{orb}}=\sum_{i \mu \nu} \epsilon_{\mu}{ }^{\nu} x_{i}^{\mu} p_{\nu}^{i} .
$$

In the absence of any internal degrees of freedom, this would be the end of the story. However, when rotations act nontrivially on the internal indices in Eq. (8), we have that rotations are generated by the total angular momentum

$$
L \equiv L_{\text {orb }}+L_{\text {int }},
$$

where $L_{\text {int }}$ is an $N_{L} \times N_{L}$ matrix acting on the internal degrees of freedom. As an example, for a spin-1/2 particle in two dimensions, the internal angular momentum operator $L_{\text {int }}=\sigma_{z} / 2$ is the generator of rotations about the $z$ axis.

The antisymmetric part of the strain generator in Eq. (11) gives only the orbital angular momentum by construction. Thus, following Ref. [36], we seek a modified strain generator $\mathcal{J}^{\mu}{ }_{\nu}$ satisfying 


$$
\sum_{\mu \nu} \epsilon_{\mu}{ }^{\nu} \mathcal{J}^{\mu}{ }_{\nu}=-L_{\text {orb }}-L_{\text {int }} .
$$

In order for this modified strain generator to be computable as a moment of the momentum density, we can define the momentum density to be

$$
g_{\mu}(\mathbf{r}) \equiv g_{\mathrm{kin}, \mu}+\frac{1}{2} \sum_{i \nu} \epsilon_{\mu}{ }^{\nu} \partial_{\nu} \delta\left(\mathbf{r}-\mathbf{x}_{i}\right) L_{\mathrm{int}}
$$

This definition is the minimal modification of the momentum density, which enforces Eq. (18). Note, importantly, that this redefinition does not change the total linear momentum,

$$
\mathbf{P}=\int d^{2} r \mathbf{g}(\mathbf{r})=\int d^{2} r \mathbf{g}_{\text {kin }}(\mathbf{r}) .
$$

By examining the first moment of the modified momentum density, we can express the modified strain generators in terms of the kinetic strain generators as

$$
\mathcal{J}^{\mu}{ }_{\nu}=-\int d^{2} r r^{\mu} g_{\nu}(\mathbf{r})=J^{\mu}{ }_{\nu}-\frac{1}{2} \epsilon^{\mu}{ }_{\nu} L_{\text {int }} .
$$

Inserting the modified momentum density into the continuity equation (2), Fourier transforming, and taking the long-wavelength limit, we find that the modified integrated stress tensor is

$$
T_{\mathrm{B}, \nu}^{\mu}=-i\left[H, \mathcal{J}^{\mu}{ }_{\nu}\right]=T_{\nu}^{\mu}+\frac{i}{2} \epsilon^{\mu}{ }_{\nu}\left[H, L_{\mathrm{int}}\right],
$$

where the subscript " $B$ " refers to Belinfante [38], for reasons we will make precise below. Because the second term in Eq. (22) originates from the internal angular momentum, we refer to it as the spin stress.

So far, we have made no assumption on the rotational symmetry of the system under study. The criterion of rotational symmetry of the Hamiltonian can be expressed as

$$
[L, H]=\left[L_{\text {orb }}, H\right]+\left[L_{\text {int }}, H\right]=0 .
$$

Combining this with Eq. (22), we see that for rotationally invariant systems, the modified stress tensor $T_{\mathrm{B}, \nu}^{\mu}$ satisfies

$$
\sum_{\mu \nu} T_{\mathrm{B}, \nu}^{\mu} \epsilon_{\mu}{ }^{\nu}=0
$$

In other words, the stress tensor corresponding to the momentum density, Eq. (19), is symmetric in flat space (where we can raise and lower indices with impunity). In the special case of spin-1/2 Dirac electrons, it was shown that the stress defined in this way is precisely the Belinfante-Rosenfeld stress tensor [36,38-40]; here, we have generalized this result to arbitrary representations of internal rotations.

Going further, we can also show that if we compare this treatment with the field-theoretic formalism of Ref. [35], we see that the strain generators $\mathcal{J}^{\mu}{ }_{\nu}$ implement precisely the generalized Belinfante procedure described there. To see this result, we can imagine defining a second-quantized version of Eq. (8), valid on an arbitrary curved surface. The action for this system takes the general form

$$
S=\int d^{3} x \sqrt{g} \psi^{\dagger}\left[i e_{0}^{t}\left(\partial_{t}-i \omega_{t} L_{\mathrm{int}}\right)-H(\vec{e}, \vec{\omega})\right] \psi,
$$

where $\psi$ is an $N_{L}$ component annihilation operator, $\vec{e}$ are frame fields (vielbeins), and $\vec{\omega}$ is the spin connection. We have defined $H(\vec{e}, \vec{\omega})$ to be the Hamiltonian with all indices covariantly contracted with vielbeins, and all derivatives replaced by (rotationally) covariant derivatives. For the sake of this discussion, we have included in the Hamiltonian all terms that involve a spatial derivative of the fermion fields. Following Refs. [35,41,42], the stress tensor and momentum density for this system can be derived by considering the conservation law associated with diffeomorphism invariance of the action. A priori, the spin connection and the vielbeins are treated as independent variables; doing so leads to the conservation law for the canonical stress tensor analogous to Eq. (12). However, if we demand that the torsion of space

$$
\vec{T}=d \vec{e}+\vec{\omega} \wedge \vec{e}
$$

remains fixed (more properly, as fixed as possible) as we vary the background geometry, then the spin connection can be expressed in terms of the vielbeins. Eliminating $\vec{\omega}$ from Eq. (25) and applying Noether's theorem yields a stress tensor that is symmetric when the system is rotationally invariant. To check whether this field-theoretic Belinfante procedure yields the same stress tensor $T_{\mathrm{B}, \nu}^{\mu}$ obtained from the improved strain generators, it suffices to consider the field-theoretic Belinfante momentum density. The correction to the momentum density from the vielbein dependence of the spin connection can be written

$$
\delta g_{\mu}=-\frac{1}{\sqrt{g}} \sum_{A, \aleph=0}^{2} \int d^{3} x \sqrt{g} \frac{\delta S}{\delta \omega_{\aleph}} \frac{\delta \omega_{\aleph}}{\delta e_{0}^{A}} e_{\mu}^{A},
$$

where $A$ and $\boldsymbol{\aleph}$ are internal (frame) and ambient spacetime indices, respectively. Taking the variations using the expressions in the Appendix A of Ref. [35] for the spin connection and evaluating the result in the unstrained geometry yields

$$
\delta g_{\mu}=\sum_{\nu} \epsilon_{\mu}{ }^{\nu} \partial_{\nu}\left(\psi^{\dagger}(\mathbf{r}) L_{\mathrm{int}} \psi(\mathbf{r})\right),
$$


which is exactly the second-quantized form of the internal momentum in Eq. (19). Thus, we conclude that the stress tensor, Eq. (22), coincides with the usual Belinfante procedure, at least on the physical Hilbert space (recall that the Belinfante stress tensor as a quantum operator is only symmetric when the equations of motion are applied, i.e., when acting on physical states). The importance of considering the Belinfante tensor will be illustrated in Sec. VI when we reexamine the viscosity of multicomponent lattice tightbinding systems, first considered in Ref. [30]. In that work, the spin connection $\vec{\omega}$ is explicitly set to zero, and hence those authors use the canonical stress tensor.

It is important to note also that there is a fundamental difference between the Belinfante procedure in relativistic and nonrelativistic systems. Because of Lorentz symmetry, in relativistic systems the Belinfante symmetrization adds a divergence-free term to the entire energy-momentum tensor. As such, integrated quantities such as the tensor $T^{\mu}{ }_{\nu}$ do not change when the stress tensor is symmetrized. By contrast, in nonrelativistic systems, there is no symmetry relating the momentum density to the stress tensor; a consequence of this result is that the integrated Belinfante tensor $T_{\mathrm{B}, \nu}^{\mu}$ is physically distinct from the integrated canonical stress tensor.

Furthermore, note that the internal angular-momentum contribution to the momentum density originates solely from the time-derivative term in the action, Eq. (25). As such, even when the Hamiltonian breaks rotational symmetry explicitly, the form of the momentum density operator is unchanged. From this result, we conclude that the stress tensor, Eq. (22), is the meaningful extension of the Belinfante "symmetrization" to nonrotationally invariant situations, validating the conjecture of Ref. [36].

However, we cannot get away with modifying the momentum density without paying some price; while the kinetic strain generators $J^{\mu}{ }_{\nu}$ generate the algebra $\mathfrak{g l}(2, \mathbb{R})$,

$$
i\left[J_{\nu}^{\mu}, J_{\rho}^{\lambda}\right]=\delta_{\rho}^{\mu} J_{\nu}^{\lambda}-\delta_{\nu}^{\lambda} J_{\rho}^{\mu},
$$

of diffeomorphism of the plane, the same cannot be said of the modified strain generators. In fact, because we have chosen the internal degrees of freedom to be invariant under shears and dilatations, and to transform only under rotations, we have that

$$
i\left[\mathcal{J}^{\mu}{ }_{\nu}, \mathcal{J}^{\lambda}{ }_{\rho}\right]=\delta_{\rho}^{\mu} J_{\nu}^{\lambda}-\delta_{\nu}^{\lambda} J_{\rho}^{\mu} .
$$

The modified strain algebra thus does not close. Furthermore, even if we were to let the internal degrees of freedom transform under shears and dilatations, the algebra of the $\mathcal{J}$ operators cannot close as long as the number of internal degrees of freedom is finite. This is because the Lie group $G L(d, \mathbb{R})$ is noncompact and hence has no finite-dimensional unitary representations [43].
We thus see that, by modifying the strain generators as per Eq. (19), we obtain the Belinfante stress tensor $T_{\mathrm{B}, \nu}^{\mu}$. Even in the absence of rotational symmetry, the Belinfante stress gives the conserved current associated with deformations of spacetime at fixed (reduced) torsion. In this way, we can fix the definition of the antisymmetric part of the stress tensor in rotationally noninvariant systems. By focusing on the Belinfante stress, we ensure that the torque density $\epsilon_{\mu}{ }^{\nu} T_{\mathrm{B}, \nu}^{\mu}$ gives only the torque due to rotational symmetry breaking in the Hamiltonian. Thus, the modified strain generators $\mathcal{J}^{\mu}{ }_{\nu}$ and Belinfante stress tensor $T_{\mathrm{B}, \nu}^{\mu}$ are the natural objects to consider in the study of viscosity of anisotropic systems.

Using this Belinfante formalism, we can proceed to calculate the viscosity tensor for arbitrary anisotropic continuum systems. First, however, let us recall that in condensed matter systems the most common source of rotational symmetry breaking comes from an underlying lattice. In order to consistently treat the viscosity in such systems (even in the low-energy $\mathbf{k} \cdot \mathbf{p}$ limit), we first develop a formalism for computing momentum transport in lattice models.

\section{CAN WE GENERALIZE TO A LATTICE SYSTEM?}

At first sight, the idea of quantifying momentum transport in a lattice system is fraught with difficulties. First and foremost, in the presence of translational symmetry breaking, total momentum is no longer conserved. This result creates difficulties in defining a continuity equation for the density of momentum: The split between external forces $\mathbf{f}^{\text {ext }}$ and internal forces,

$$
f_{\nu}^{\text {int }} \equiv-\sum_{\mu} \partial_{\mu} \tau_{\nu}^{\mu},
$$

becomes unnatural. Furthermore, the strain formalism of Ref. [37] and Sec. II is no longer available if we work with a lattice-regularized system with discrete positions since the momentum operators are no longer well defined.

To remedy these issues, in this section we generalize the strain and stress formalisms to tight-binding lattice systems and show that there exists a meaningful notion of stress tensor and momentum transport, which reduces to those of Sec. II in an appropriate continuum limit. Our motivation for developing this formalism is twofold. First, a lattice formulation allows us to incorporate rotational symmetry breaking into the viscosity formalism in a controlled way, where the strength of the anisotropy can be quantitatively tied to the underlying crystal structure. Second, we provide a framework for interpreting and understanding previous results on the viscosity of lattice systems such as Chern insulators [30] and the connection to low-energy expansions of the viscosity near multiple Fermi pockets such as in graphene [36]. 
To begin, let us consider a general lattice Hamiltonian

$$
\begin{aligned}
H= & \sum_{\mathbf{R} \mathbf{R}^{\prime} n m} c_{n \mathbf{R}}^{\dagger} f^{n m}\left(\mathbf{R}, \mathbf{R}^{\prime}\right) c_{m \mathbf{R}^{\prime}} \\
& +\frac{1}{2} \sum_{\mathbf{R} \mathbf{R}^{\prime} p l n m}\left[V\left(\mathbf{R}, \mathbf{R}^{\prime}\right)\right]^{n m p \ell} c_{n \mathbf{R}}^{\dagger} c_{m \mathbf{R}^{\prime}}^{\dagger} c_{p \mathbf{R}} c_{\ell \mathbf{R}^{\prime}},
\end{aligned}
$$

where we have, for convenience, introduced a secondquantized description. Here, $\mathbf{R}$ and $\mathbf{R}^{\prime}$ are lattice vectors in a Bravais lattice, and the operators $c_{n \mathbf{R}}^{\dagger}$ create a fermion in unit cell $\mathbf{R}$ in a state indexed by $n$. The fermion operators satisfy the anticommutation relations

$$
\begin{gathered}
\left\{c_{n \mathbf{R}}, c_{m \mathbf{R}^{\prime}}\right\}=0, \\
\left\{c_{n \mathbf{R}}^{\dagger}, c_{m \mathbf{R}^{\prime}}\right\}=\delta_{\mathbf{R}^{\prime}} \delta_{n m} .
\end{gathered}
$$

Although we consider a fermionic system for concreteness, the generalization of all of our results to bosonic systems is straightforward. Note that the states created by $c_{n \mathbf{R}}^{\dagger}$ for the same $\mathbf{R}$ need not all be centered at the same spatial location; nevertheless, for simplicity, we refer to the degrees of freedom indexed by $n$ as "internal" degrees of freedom. The first term $f^{n m}\left(\mathbf{R}, \mathbf{R}^{\prime}\right)$ is the single-particle Hamiltonian, and it includes both kinetic and onsite interactions. The second term $\left[V\left(\mathbf{R}, \mathbf{R}^{\prime}\right)\right]^{n m p \ell}$ is the (normal-ordered) two-body interaction energy. We assume that the Hamiltonian has discrete translation symmetry,

$$
\begin{gathered}
f^{n m}\left(\mathbf{R}+\mathbf{R}_{a}, \mathbf{R}^{\prime}+\mathbf{R}_{a}\right)=f^{n m}\left(\mathbf{R}, \mathbf{R}^{\prime}\right), \\
{\left[V\left(\mathbf{R}+\mathbf{R}_{a}, \mathbf{R}^{\prime}+\mathbf{R}_{a}\right)\right]^{n m p \ell}=\left[V\left(\mathbf{R}, \mathbf{R}^{\prime}\right)\right]^{n m p \ell},}
\end{gathered}
$$

where

$$
\mathbf{R}_{a}=\sum_{\mu} n_{a}^{\mu} \mathbf{a}_{\mu}, \quad n_{a}^{\mu} \in \mathbb{Z}
$$

is any Bravais lattice vector, written in terms of the primitive lattice vectors $\mathbf{a}_{\mu}$. Beyond this assumption, we make no other assumptions about the symmetry. Because of the preponderance of expressions with repeated indices that appear as we consider lattice systems, we remind the reader that we will not sum over repeated indices unless noted explicitly. Let us also introduce a (normalized) basis $\left\{\mathbf{b}^{\mu} \mid \mu=1,2\right\}$ for the reciprocal lattice satisfying

$$
\mathbf{a}_{\nu} \cdot \mathbf{b}^{\mu}=\delta_{\nu}^{\mu} .
$$

In order to discuss momentum transport in this system, we must first define a lattice momentum density. We define it in such a way that we recover the continuum momentum density, Eq. (19), in the long-wavelength limit, which is equivalently the limit that the lattice spacing $\left|\mathbf{a}_{\mu}\right|$ goes to zero, keeping the ratios $\left|\mathbf{a}_{\mu}\right| /\left|\mathbf{a}_{\nu}\right|$ fixed. Furthermore, we expect a hydrodynamic approach to be valid only in a "coarse-grained" sense, which means that we should focus primarily on the transport of momentum between unit cells (rather than within the unit cell). As we emphasized in $\mathrm{Sec}$. II, it is also critical for us to incorporate internal angular momentum into the momentum density. In the lattice system, the internal angular momentum is determined by the representation of rotations on the internal degrees of freedom $\{n\}$; systems with the same Hamiltonian $H$ may have different internal rotation generators $L_{n m}^{\text {int }}$ depending on the physical meaning (embedding) of the degrees of freedom $\{n\}$.

Taking these considerations into account, we decompose the lattice momentum density

$$
g_{\mu}^{\mathrm{L}}(\mathbf{R})=g_{\mu}^{\mathrm{kin}}(\mathbf{R})+g_{\mu}^{\mathrm{int}}(\mathbf{R})
$$

into a kinetic piece $g_{\mu}^{\mathrm{kin}}(\mathrm{R})$ and an internal angularmomentum contribution $g_{\mu}^{\text {int }}(\mathrm{R})$. For the kinetic momentum, we write

$$
\begin{aligned}
g_{\mu}^{\mathrm{kin}}(\mathbf{R})= & \frac{i}{4\left|\mathbf{a}_{\mu}\right|} \sum_{n}\left(c_{n \mathbf{R}+\mathbf{a}_{\mu}}^{\dagger} c_{n \mathbf{R}}-c_{n \mathbf{R}-\mathbf{a}_{\mu}}^{\dagger} c_{n \mathbf{R}}+c_{n \mathbf{R}}^{\dagger} c_{n \mathbf{R}-\mathbf{a}_{\mu}}\right. \\
& \left.-c_{n \mathbf{R}}^{\dagger} c_{n \mathbf{R}+\mathbf{a}_{\mu}}\right) .
\end{aligned}
$$

Similarly, for the internal momentum density, we take

$$
\begin{aligned}
g_{\mu}^{\mathrm{int}}(\mathbf{R})= & \sum_{n m \nu} \frac{1}{4\left|\mathbf{a}_{\nu}\right|} \tilde{\epsilon}_{\mu}{ }^{\nu} L_{\mathrm{int}}^{n m}\left(c_{n \mathbf{R}+\mathbf{a}_{\nu}}^{\dagger} c_{m \mathbf{R}}-c_{n \mathbf{R}-\mathbf{a}_{\nu}}^{\dagger} c_{m \mathbf{R}}\right. \\
& \left.+c_{n \mathbf{R}}^{\dagger} c_{m} \mathbf{R}+\mathbf{a}_{\nu}-c_{n \mathbf{R}}^{\dagger} c_{m} \mathbf{R}-\mathbf{a}_{\nu}\right),
\end{aligned}
$$

where we have defined the lattice epsilon tensor as

$$
\tilde{\epsilon}_{\mu}{ }^{\nu} \equiv \mathbf{a}_{\mu}^{\mathrm{T}} \cdot \mathbf{\epsilon} \cdot \mathbf{b}^{\nu} .
$$

(Note that for systems with threefold rotational symmetry, contractions of lattice vector indices are not tensorial. To make contact with standard results in these cases, every index should be projected onto the vector representation of the symmetry group. We leave these insertions implicit to avoid overburdening notation.) The sum of fermion bilinears appearing here gives a symmetric discretization of the derivative. As such, in the limit of small lattice spacing $\left|\mathbf{a}_{\mu}\right| \rightarrow 0$, the sum of Eqs. (40) and (41) coincides with the continuum momentum density, Eq. (19),

$$
\lim _{|\mathbf{a}| \rightarrow 0} g_{\mu}^{\mathrm{L}}(\mathbf{R})=\hat{\mathbf{a}}_{\mu} \cdot \mathbf{g}(\mathbf{R}) .
$$

Additionally, the particular discretization of the derivative that we have chosen here includes all terms that carry momentum into or out of the unit cell $\mathbf{R}$ from adjacent unit cells. This case is reminiscent of the form of conserved 
currents in lattice gauge theories [44] and is necessary to ensure that we recover the proper long-wavelength properties upon coarse graining.

Taking Eq. (39) as our starting point, we can now examine its time derivative and attempt to derive an analogue of Eq. (2). This method allows us to extract the integrated Belinfante stress tensor. Since we are primarily interested in the integrated stress tensor, we proceed in momentum space. We introduce the momentum space creation and annihilation operators

$$
\begin{gathered}
c_{n \mathbf{k}}=\sum_{\mathbf{R}} c_{n \mathbf{R}} e^{i \mathbf{k} \cdot \mathbf{R}}, \\
c_{n \mathbf{k}}^{\dagger}=\sum_{\mathbf{R}} c_{n \mathbf{R}}^{\dagger} e^{-i \mathbf{k} \cdot \mathbf{R}} .
\end{gathered}
$$

Inserting these into Eqs. (40) and (41), we find

$$
g_{\mu}^{\mathrm{L}}(\mathbf{R})=\sum_{\mathbf{q}} e^{i \mathbf{q} \cdot \mathbf{R}}\left[g_{\mu}^{\mathrm{kin}}(\mathbf{q})+g_{\mu}^{\mathrm{int}}(\mathbf{q})\right],
$$

with

$$
g_{\mu}^{\mathrm{kin}}(\mathbf{q})=\frac{1}{2\left|\mathbf{a}_{\mu}\right|} \sum_{n \mathbf{k}}\left[\sin (\mathbf{k}+\mathbf{q}) \cdot \mathbf{a}_{\mu}+\sin \mathbf{k} \cdot \mathbf{a}_{\mu}\right] c_{n \mathbf{k}}^{\dagger} c_{n \mathbf{k}+\mathbf{q}}
$$

and

$$
\begin{aligned}
g_{\mu}^{\text {int }}(\mathbf{q})= & \sum_{\mathbf{k} \nu, m, n} \frac{i}{2\left|\mathbf{a}_{\nu}\right|} \tilde{\epsilon}_{\mu}{ }^{\nu}\left[\sin (\mathbf{k}+\mathbf{q}) \cdot \mathbf{a}_{\nu}-\sin \mathbf{k} \cdot \mathbf{a}_{\nu}\right] \\
& \times L_{\text {int }}^{n m} c_{n \mathbf{k}}^{\dagger} c_{m \mathbf{k}+\mathbf{q}} .
\end{aligned}
$$

Note that, in this form, we see clearly that the total lattice momentum, obtained by taking $\mathbf{q} \rightarrow 0$, coincides with the conventional expression for the momentum operator found in, e.g., Ref. [45]. In particular, the internal angular momentum does not contribute to the total momentum, as in the continuum case.

By taking the commutator of Eqs. (47) and (48), with the Hamiltonian (32) we can attempt to extract the stress tensor and external force densities from the time derivative

$$
\partial_{t} g_{\mu}^{\mathrm{L}}(\mathbf{q})=i\left[H, g_{\mu}^{\mathrm{L}}(\mathbf{q})\right],
$$

where $H$ is the Hamiltonian (32). Our goal is to make an analogy with the continuum continuity equation in the long-wavelength $\mathbf{q} \rightarrow 0$ limit. To do so, it is simplest to expand Eqs. (47) and (48) to leading order in $\mathbf{q}$, which gives

$$
\begin{aligned}
g_{\mu}^{\mathrm{L}}(\mathbf{q})= & P_{\mu}+\frac{1}{2} \sum_{n m \nu \mathbf{k}} q_{\nu} c_{n \mathbf{k}}^{\dagger}\left[\left\{\frac{\sin \mathbf{k} \cdot \mathbf{a}_{\mu}}{\left|\mathbf{a}_{\mu}\right|}, \partial^{\nu}\right\} \delta^{n m}\right. \\
& \left.+i \tilde{\epsilon}_{\mu}{ }^{\nu} \cos \mathbf{k} \cdot \mathbf{a}_{\nu} L_{\text {int }}^{n m}\right] c_{m \mathbf{k}}+\ldots,
\end{aligned}
$$

where we have introduced the total momentum operator $\mathbf{P}=\mathbf{g}^{\mathrm{L}}(\mathbf{q}=0)$; here, $\partial^{\nu}$ represents a derivative with respect to $k_{\nu} \equiv \mathbf{k} \cdot \hat{\mathbf{a}}_{\nu}$ (we expect no confusion to arise between the use of $\partial$ for momentum and position derivatives-position derivatives always carry a lower index and momentum derivatives an upper index). Examining the expression in brackets, we recognize that it is a discretized form of the strain generator Eq. (21). In particular, if we define

$$
\begin{aligned}
\mathcal{J}_{\mathrm{L}, \nu}^{\mu} \equiv & -\frac{i}{2} \sum_{\mathbf{k} n m} c_{n \mathbf{k}}^{\dagger}\left[\left\{\frac{\sin \mathbf{k} \cdot \mathbf{a}_{\nu}}{\left|\mathbf{a}_{\nu}\right|}, \frac{\partial}{\partial k_{\mu}}\right\} \delta^{n m}\right. \\
& \left.+i \tilde{\epsilon}_{\nu}{ }^{\mu} \cos \mathbf{k} \cdot \mathbf{a}_{\mu} L_{\mathrm{int}}^{n m}\right] c_{m \mathbf{k}},
\end{aligned}
$$

then we have

$$
g_{\mu}^{\mathrm{L}}(\mathbf{q})=P_{\mu}+i \sum_{\nu} q_{\nu} \mathcal{J}_{\mathrm{L}, \mu}^{\nu}+\mathcal{O}\left(\mathbf{q}^{2}\right) .
$$

Comparing with the continuity equation, this result allows us to identify, in the long-wavelength limit,

$$
\begin{gathered}
f_{\mu}^{\mathrm{ext}}=-i\left[H, P_{\mu}\right], \\
T_{\mathrm{B}, \nu}^{\mu}=-i\left[H, \mathcal{J}_{\mathrm{L}, \nu}^{\mu}\right] .
\end{gathered}
$$

Note that, unlike in a continuum system, the external force $f_{\mu}^{\text {ext }}$ need not vanish for the Hamiltonian (32) because the interaction term $\left[V\left(\mathrm{R}, \mathrm{R}^{\prime}\right)\right]^{n m p l}$ is not invariant under infinitesimal translations. More physically, $f_{\mu}^{\text {ext }}$ corresponds to the rate of momentum relaxation due to Umklapp scattering [46]. Note also that all $\mathrm{O}\left(\mathbf{q}^{2}\right)$ contributions to the lattice momentum density can, in the longwavelength limit, be written as

$$
g_{\mu}^{\mathrm{L}}(\mathbf{q})=P_{\mu}+i \sum_{\nu} q_{\nu} \mathcal{J}_{\mathrm{L}, \mu}^{\nu}+\sum_{\nu} i q_{\nu} M_{\mu}^{\nu}(\mathbf{q})
$$

for some $\mathbf{q}$-dependent tensor $M^{\nu}{ }_{\mu}(\mathbf{q})$ that vanishes at least linearly as $\mathbf{q} \rightarrow 0$. This tensor is, in principle, computable by Taylor expanding Eqs. (47) and (48). By taking the time derivative of $M^{\mu}{ }_{\nu}$, we can compute the long-wavelength stress tensor $\tau_{\mathrm{B}, \nu}^{\mu}(\mathbf{q})$ to arbitrary order in $\mathbf{q}$.

For interacting systems, defining the stress tensor and internal forces in this way is tantamount to making a particular choice for how to split the force density between internal and external contributions. Although there is no ambiguity in attributing uniform forces to $\mathbf{f}^{\text {ext }}$, solely to the interaction term via Eq. (53), at higher order in $\mathbf{q}$, both viscous and external forces may appear as divergences of functions. Our formalism following from Eq. (55) chooses to attribute all nonuniform forces to internal forces, Eq. (31). This nonuniqueness is at the heart of the claim that the viscosity requires translational invariance to be well defined [7,37]. Nevertheless, we see that in the longwavelength limit, there exists a natural choice for the 
integrated stress tensor and hence a natural definition for the viscosity and elastic moduli tensors.

Finally, we point out that, due to the factor of $\cos \mathbf{k} \cdot \mathbf{a}_{\mu}$ multiplying the internal angular momentum in Eq. (51), the internal angular-momentum contribution to the lattice stress tensor is not purely antisymmetric, in contrast to the continuum case. Mathematically, this factor originated from our choice of symmetric discretization of the derivative in Eq. (41). Physically, it reflects the fact that $\mathcal{J}_{\mathrm{L}, \nu}^{\mu}$ is not a generator of simple uniform deformations of the plane beyond leading order in $\mathbf{k} \cdot \mathbf{a}$, which can be seen by expanding the kinetic strain.

By carrying out the commutator in Eq. (54), we can derive a general expression for the lattice stress tensor. It is beneficial to carry this computation out in two parts-first for the noninteracting contribution to the stress, and second for the interaction contribution. Introducing the Fourier transform

$$
f^{n m}(\mathbf{k})=\sum_{\mathbf{R}} e^{-i \mathbf{k} \cdot \mathbf{R}} f^{n m}(\mathbf{R}, 0)
$$

of the single-particle Hamiltonian in Eq. (32), we find rather directly that the single-particle contribution $T_{\mathrm{B} 0, \nu}^{\mu}$ to the stress tensor is given by

$$
\begin{aligned}
T_{\mathrm{B} 0, \nu}^{\mu}= & \sum_{n m \mathbf{k}} c_{n \mathbf{k}}^{\dagger}\left(\partial^{\mu} f_{n m}(\mathbf{k}) \frac{\sin \mathbf{k} \cdot \mathbf{a}_{\nu}}{\left|\mathbf{a}_{\nu}\right|}\right. \\
& \left.+\frac{i}{2} \tilde{\epsilon}^{\mu}{ }_{\nu} \cos \mathbf{k} \cdot \mathbf{a}_{\mu}\left[f(\mathbf{k}), L_{\mathrm{int}}\right]_{n m}\right) c_{m \mathbf{k}} .
\end{aligned}
$$

Combined with Eq. (54), we see from this result that in a noninteracting system, lattice effects enter only via the anisotropy of the stress tensor.

Next, we compute the interaction contributions to the lattice stress tensor. The algebra is a bit more involved, and it is most convenient to use the representation

$$
\mathcal{J}_{\mathrm{L}, \nu}^{\mu}=-\sum_{R} R^{\mu} g_{\nu}(\mathbf{R})
$$

for the lattice strain generator. Defining the shorthand

$$
\begin{aligned}
V_{\mathbf{R}}^{\mathbf{k q}} & \equiv \sum_{\mathbf{R}^{\prime} n m p \ell}\left[V\left(\mathbf{R}^{\prime}, \mathbf{R}\right)\right]^{n m p \ell} c_{n \mathbf{R}^{\prime}}^{\dagger} c_{m \mathbf{k}}^{\dagger} c_{p \mathbf{R}^{\prime}} c_{\ell \mathbf{k}+\mathbf{q}}, \\
(V L)_{\mathbf{R}}^{\mathbf{k q}} & =\sum_{\mathbf{R}^{\prime} n m p \ell n^{\prime}}\left[V\left(\mathbf{R}^{\prime}, \mathbf{R}\right)\right]^{n m p n^{\prime}} L_{\text {int }}^{n^{\prime} \ell} c_{n \mathbf{R}^{\prime}}^{\dagger} c_{m \mathbf{k}}^{\dagger} c_{p \mathbf{R}^{\prime}} c_{\ell \mathbf{k}+\mathbf{q}},
\end{aligned}
$$

$$
(L V)_{\mathbf{R}}^{\mathbf{k q}}=\sum_{\mathbf{R}^{\prime} n m p \ell n^{\prime}} L_{\mathrm{int}}^{m n^{\prime}}\left[V\left(\mathbf{R}^{\prime}, \mathbf{R}\right)\right]^{n n^{\prime} p \ell} c_{n \mathbf{R}^{\prime}}^{\dagger} c_{m \mathbf{k}}^{\dagger} c_{p \mathbf{R}^{\prime}} c_{\ell \mathbf{k}+\mathbf{q}},
$$

we find that $T_{\mathrm{BIK}, \nu}^{\mu}$, the lattice form of the BelinfanteIrving-Kirkwood stress tensor [47], is

$$
\begin{aligned}
T_{\mathrm{BIK}, \nu}^{\mu}= & \sum_{\mathbf{R} \mathbf{k q}} \frac{1}{4\left|\mathbf{a}_{\nu}\right|} R^{\mu} e^{i \mathbf{q} \cdot \mathbf{R}}\left[\left(V_{\mathbf{R}+\mathbf{a}_{\nu}}^{\mathbf{k q}}-V_{\mathbf{R}}^{\mathbf{k q}}\right)\left(e^{i(\mathbf{k}+\mathbf{q}) \cdot \mathbf{a}_{\nu}}+e^{-i \mathbf{k} \cdot \mathbf{a}_{\nu}}\right)-\left(V_{\mathbf{R}-\mathbf{a}_{\nu}}^{\mathbf{k q}}-V_{\mathbf{R}}^{\mathbf{k q}}\right)\left(e^{-i(\mathbf{k}+\mathbf{q}) \cdot \mathbf{a}_{\nu}}+e^{i \mathbf{k} \cdot \mathbf{a}_{\nu}}\right)\right] \\
& +\sum_{\mathbf{R} \mathbf{k q} \lambda} \frac{i}{4\left|\mathbf{a}_{\lambda}\right|} R^{\mu} \tilde{\epsilon}_{\nu}^{\lambda} e^{i \mathbf{q} \cdot \mathbf{R}}\left\{e^{i \mathbf{k} \cdot \mathbf{a}_{\lambda}}\left[(L V)_{\mathbf{R}}^{\mathbf{k q}}-(V L)_{\mathbf{R}-\mathbf{a}_{\lambda}}^{\mathbf{k q}}\right]+e^{-i \mathbf{k} \cdot \mathbf{a}_{\lambda}}\left[(V L)_{\mathbf{R}+\mathbf{a}_{\lambda}}^{\mathbf{k q}}-(L V)_{\mathbf{R}}^{\mathbf{k q}}\right]\right. \\
& \left.+e^{i(\mathbf{k}+\mathbf{q}) \cdot \mathbf{a}_{\lambda}}\left[(V L)_{\mathbf{R}}^{\mathbf{k q}}-(L V)_{\left.\mathbf{R}+\mathbf{a}_{\lambda}\right]}^{\mathbf{k q}}\right]+e^{-i(\mathbf{k}+\mathbf{q}) \cdot \mathbf{a}_{\lambda}}\left[(L V)_{\mathbf{R}-\mathbf{a}_{\lambda}}^{\mathbf{k q}}-(V L)_{\mathbf{R}}^{\mathbf{k q}}\right]\right\} .
\end{aligned}
$$

In deriving this expression, we have exploited the translational and exchange symmetries of the interaction $\left[V\left(\mathbf{R}, \mathbf{R}^{\prime}\right)\right]^{n m p \ell}$ in order to obtain a somewhat compact expression. Nevertheless, we will spare the reader any explicit use of Eq. (62) going forward.

\section{A. Addition of a rational magnetic flux}

Here, we remark on the addition of a magnetic field $B$ of rational flux $\phi=p / q$ (in units of the flux quantum $\phi_{0}$ ) to our formalism for lattice elasticity. After a Peierls substitution, our formalism as it stands, with $L_{\text {int }}=0$ (the oneband case), reproduces the results of Refs. [30,31] for the Hofstadter model on a square lattice [48]. Within our formalism, it is the lattice vectors' (enlarged) magnetic unit cells that go into defining the lattice momentum, Eq. (39).
The only subtlety in the more general case is to ensure that the generator of internal angular momentum $\hat{L}_{\text {int }}$ is not artificially modified by the addition of the magnetic field. As the creation operators, annihilation operators, and Hamiltonian increase in dimension proportional to $q$ when the Brillouin zone is replaced by the contracted magnetic Brillouin zone, we must ensure that $L_{\text {int }}=L_{\text {int }}(B \rightarrow 0) \otimes$ $\mathbb{I}_{p \times p}$ - the internal angular momentum is determined in the zero-field limit (corresponding to spin or pseudospin degrees of freedom) and is trivial (block diagonal) in the space of the magnetic subbands. With these caveats in mind, the application of our formalism to a multiband lattice system in the presence of a magnetic field is straightforward. Furthermore, all the general considerations of this and the following sections will continue to hold in the presence of a magnetic field. 


\section{B. Connecting the lattice to the continuum}

With this method of defining the momentum density and long-wavelength stress tensor, we see that the lattice forces enter into hydrodynamics only through Umklapp scattering in interacting systems. In a noninteracting system, the total momentum $\mathbf{P}$ defined here is a conserved quantity. Thus, in our formalism, we find that hydrodynamics applies when the impurity scattering rate (here set to zero from the outset) and the Umklapp scattering rate go to zero. This observation, along with the similarities between Eqs. (21) and (51), suggests a concrete connection between the lattice and continuum formulations.

We must be careful, however, to distinguish two different points of view on the continuum limit. In the first, which we call the "lattice gauge theory" point of view, the lattice Hamiltonian, Eq. (32), represents a regularized UV completion of some underlying continuum theory. This perspective was taken in Ref. [30], where the lattice model for a Chern insulator was interpreted as the UV completion of a theory of free Dirac fermions. In this point of view, the continuum model is recovered by taking the appropriate $\left|\mathbf{a}_{\mu}\right| \rightarrow 0$ limit. This perspective is also appropriate for metallic systems (such as graphene [36]), where we can view the $|\mathbf{a}| \rightarrow 0$ limit of Eq. (32) as reproducing lowenergy Fermi pockets with anisotropic dispersion. In the lattice gauge theory point of view, we see that as $\left|\mathbf{a}_{\mu}\right| \rightarrow 0$, the free-fermion integrated stress tensor becomes

$$
\begin{aligned}
T_{\mathrm{B} 0, \nu}^{\mu} & \rightarrow \sum_{n m \mathbf{k}} c_{\mathbf{k} n}^{\dagger}\left(k_{\nu} \partial^{\mu} f_{n m}^{0}(\mathbf{k})+\frac{i}{2} \tilde{\epsilon}^{\mu}{ }_{\nu}\left[f^{0}(\mathbf{k}), L_{\mathrm{int}}\right]_{n m}\right) c_{m \mathbf{k}}, \\
& =\hat{\mathbf{b}}^{\mu} \cdot \mathbf{T}_{\mathrm{B}}, \cdot \hat{\mathbf{a}}_{\nu}
\end{aligned}
$$

where $f^{0}$ is the continuum limit of the single-particle Hamiltonian. Equation (63) is nothing other than the continuum free-fermion stress tensor projected along the direct and reciprocal lattice directions. Similarly, the interaction contribution to the stress tensor reproduces the (Belinfante-symmetrized) Irving-Kirkwood stress tensor [37]. This result corresponds to a coarse graining where we ignore physics at the lattice scale and focus instead only on interunit cell dynamics.

However, there is a different notion of the continuum limit in condensed matter, where we view the Hamiltonian (32) as a tight-binding or Wannier approximation to an underlying continuous Schrödinger equation with a periodic potential. In this case, taking the continuum limit corresponds to leaving the lattice constant fixed but letting the number of internal degrees of freedom go to infinity, such that the operators $c_{n \mathbf{R}} \rightarrow c_{\sigma \mathbf{R}}$, with $\mathbf{r}$ a vector within the unit cell and $\sigma$ a spin index. The operator $c_{\sigma \mathbf{r R}}$ annihilates an electron in a delta-function localized orbital with spin $\sigma$ at position $\mathbf{r}+\mathbf{R}$. We refer to this method of taking the continuum limit as the tight-binding continuum limit. Examining Eqs. (57) and (62), we see that in the tight-binding continuum limit, the sums over internal degrees of freedom are replaced with real-space integrals. In this limit, the lattice momentum density, Eq. (39), is a unit-cell averaged momentum; it follows that other observables, such as the stress, correspond to unit-cell averages in this limit. We thus see in the tight-binding continuum limit that our lattice formalism is able to produce a well-defined stress tensor even in the presence of a continuous periodic potential.

When we are interested in the viscosity of a particular model, we should ask ourselves which of these continuum limits is the most appropriate for interpreting our results. The lattice gauge theory continuum limit produces a translationally invariant but potentially anisotropic system, where UV divergences from an unbounded negative spectrum have been regularized by the lattice. The tightbinding continuum limit, on the other hand, produces a system with only discrete translational symmetry and no momentum conservation. Conversely, as long as we start with a basis of Wannier functions that fully captures the low-energy behavior of our system, taking the tight-binding continuum limit should not quantitatively change any observables. This idea is not true of the lattice gauge theory continuum limit, where the continuum model has a very different set of symmetries and degrees of freedom than the lattice approximation.

As we will explore in Sec. VIB, we expect both continuum limits to give similar results for metallic systems with small Fermi pockets, where we can Taylor expand observables in $\mathbf{k}$. For band insulators, special care must be taken with the lattice gauge theory continuum limit because a band insulator is characterized by an integer filling

$$
\nu=N_{e} / N_{c}
$$

of $N_{e}$ electrons in $N_{c}$ unit cells. Rewriting the number of unit cells in terms of the total volume and the volume $V_{c}$ of a unit cell, we find that

$$
\nu=V_{c} \bar{n},
$$

where $\bar{n}$ is the particle density. Now, in the lattice-gauge continuum limit, $V_{c} \rightarrow 0$. This result presents a conundrum for an insulator, where $\nu \in \mathbb{Z}$ implies that we must also take the density $\bar{n} \rightarrow \infty$. Intuitively, this case corresponds to treating the filled bands as forming, in the limit, a uniform Dirac sea. While this perspective is useful for some purposes, it is at odds with the reality that most continuum fluids of interest have fixed, finite density. This difficulty does not arise in the tight-binding continuum limit since the unit-cell volume stays fixed. In that case, however, taking the continuum limit requires introducing additional degrees of freedom that are not contained in the original model, and there is no guarantee or expectation that such a procedure is unique. For insulators whose low-energy physics is 
dominated by a set of discrete band inversions, we can circumvent these difficulties by Taylor expanding the Hamiltonian and stress tensors around the band inversion (analogous to our discussion of metals). We will see an example of this case in Sec. VI when we examine the Chern insulator.

\section{VISCOSITY TENSOR IN ANISOTROPIC SYSTEMS}

Now that we have established a formalism for longwavelength hydrodynamics both in the continuum and on the lattice, we can examine the viscosity tensor. Recall from Eq. (5) that the viscosity $\eta$ and elastic moduli $\kappa$ govern the change in the average stress tensor,

$$
\left\langle\tau^{\mu}{ }_{\nu}\right\rangle=\left\langle\tau^{\mu}{ }_{\nu}\right\rangle_{0}-\sum_{\lambda \rho}\left[\kappa^{\mu}{ }_{\nu}{ }_{\rho}{ }_{\rho} \partial_{\lambda} u^{\rho}+\eta^{\mu}{ }_{\nu}{ }_{\rho} \partial_{\lambda} v^{\rho}+\ldots\right],
$$

where $u^{\rho}$ is a displacement field and $v^{\rho}$ is its time derivative, which gives a velocity field. We now have all the tools necessary to compute the viscosity for both lattice and continuum systems. To do this computation, in Sec. IV A, we make use of the Kubo formalism of Ref. [37]. Next, in Sec. IV B, we discuss the decomposition of the viscosity tensor into physically significant components. Finally, in Sec. IV C, we discuss symmetry constraints on these components. All of what follows will apply equally well in the continuum or on the lattice, provided the appropriate definitions of strain and stress are used.

\section{A. Kubo formalism}

We add to our Hamiltonian [either Eq. (8) or (32)] a strain perturbation

$$
H_{1}=\sum_{\mu \nu} \frac{\partial \lambda_{\mu}{ }^{\nu}}{\partial t} \mathcal{J}_{\nu}^{\mu}
$$

where $\mathcal{J}$ is the strain generator, either in the continuum or the lattice, as appropriate. The field $\partial \lambda_{\mu}{ }^{\nu} / \partial t$ represents the uniform part of a velocity gradient in the electron system. Up to a time-dependent gauge transformation [37], this perturbation gives rise to the coupling between the spatial metric (or, more generally, vielbeins) and the Belinfante tensor $T_{\mathrm{B}, \nu}^{\mu}$, which arises naturally from the field theory discussed in Sec. II. The field $\partial \lambda_{\mu}{ }^{\nu} / \partial t$ depends on time, and we write

$$
\frac{\partial \lambda_{\mu}{ }^{\nu}}{\partial t}(t)=\int d t \frac{\partial \lambda_{\mu}{ }^{\nu}}{\partial t}(\omega) e^{-i \omega t} .
$$

We then compute the linear response of the Belinfante stress $T_{\mathrm{B}, \nu}^{\mu}$. Making use of the definitions in Eqs. (22) and (54), we write

$$
\begin{aligned}
& \delta\left\langle T_{\mathrm{B}, \nu}^{\mu}\right\rangle(\omega)=\sum_{\lambda \rho} X^{\mu}{ }_{\nu}{ }_{\rho}(\omega) \frac{\partial \lambda_{\lambda}{ }^{\rho}}{\partial t}(\omega), \\
& X_{\nu}^{\mu}{ }_{\nu}{ }_{\rho}(\omega)= \frac{1}{V \omega^{+}}\left(\left\langle\left[T_{\mathrm{B}, \nu}^{\mu}, \mathcal{J}^{\lambda}{ }_{\rho}\right]\right\rangle_{0}\right. \\
&\left.+\int_{0}^{\infty} d t e^{i \omega^{+} t}\left\langle\left[T_{\mathrm{B}, \nu}^{\mu}(t), \mathcal{T}_{\mathrm{B}, \rho}^{\lambda}(0)\right]\right\rangle_{0}\right),
\end{aligned}
$$

where time evolution and averages $\langle\cdot\rangle_{0}$ are evaluated with respect to the unperturbed Hamiltonian, and $V$ is the volume of the system. Furthermore, $\omega^{+}=\omega+i \epsilon$, and it is understood that $\epsilon$ is taken to zero at the end of the calculation.

The response function $X^{\mu}{ }_{\nu}{ }_{\rho} \rho(\omega)$ incorporates both elastic moduli

$$
\kappa_{\nu}^{\mu \lambda}{ }_{\nu}^{\lambda}=\frac{1}{V}\left\langle T_{\mathrm{B}, \nu}^{\mu}\right\rangle_{0} \delta_{\rho}^{\lambda}+\lim _{\omega \rightarrow 0} i \omega X_{\nu}^{\mu}{ }_{\nu}{ }_{\rho}(\omega)
$$

and the zero-frequency viscosity tensor

$$
\eta_{\nu \rho}^{\mu}{ }_{\nu}^{\lambda}=\lim _{\omega \rightarrow 0}\left[X_{\nu \rho}^{\mu}{ }_{\nu}^{\lambda}+\frac{i}{\omega^{+}} \kappa^{\mu}{ }_{\nu \rho}^{\lambda}\right] .
$$

While this formula is valid for both dissipative and nondissipative responses, we focus here on the nondissipative Hall response

$$
\left(X^{\mathrm{H}}\right)_{\nu \rho}^{\mu}{ }_{\nu}^{\lambda} \equiv \frac{1}{2}\left(X_{\nu}^{\mu}{ }_{\nu}^{\lambda}-X_{\rho}^{\lambda}{ }_{\nu}{ }_{\nu}\right) .
$$

For the Hall response, we can use the Jacobi identity along with the Ward identity, Eq. (22), to simplify the contact term,

$$
\begin{aligned}
C_{\nu}^{\mu}{ }_{\nu}{ }^{\prime} \rho & \equiv \frac{1}{\omega} \lim _{\omega \rightarrow 0} i \omega\left(X^{\mathrm{H}}\right)_{\nu}^{\mu}{ }_{\nu \rho}^{\lambda} \\
& \left.=\frac{i}{2 \omega}\left(\left\langle\left[T_{\mathrm{B}, \nu}^{\mu}, \mathcal{J}^{\lambda}{ }_{\rho}\right]\right\rangle_{0}-\left[T_{\mathrm{B}, \rho}^{\lambda}, \mathcal{J}^{\mu}{ }_{\nu}\right]\right\rangle_{0}\right) .
\end{aligned}
$$

\section{B. Decomposition of the Hall viscosity tensor}

Before applying this Kubo formula to some model systems, let us first analyze the properties of the nondissipative response tensor. We are primarily interested in fluids, where we expect the Hall elastic moduli to vanish in the long-wavelength limit (we revisit the elastic moduli in the next section). Focusing on the Hall viscosity, without any symmetries, we have the decomposition

$$
\begin{aligned}
& \left(\eta^{\mathrm{H}}\right)_{\nu \rho}^{\mu \lambda} \equiv \frac{1}{2}\left(\eta_{\nu}^{\mu}{ }_{\nu}^{\lambda}{ }^{\lambda}-\eta^{\lambda}{ }_{\rho}^{\mu}{ }_{\nu}\right) \\
& =\eta^{\mathrm{H}}\left(\sigma^{z} \wedge \sigma^{x}\right)^{\mu}{ }_{\nu \rho}^{\lambda}+\gamma\left(\sigma^{z} \wedge \epsilon\right)^{\mu}{ }_{\nu}{ }_{\rho}{ }_{\rho}+\Theta\left(\sigma^{x} \wedge \epsilon\right)^{\mu}{ }_{\nu}{ }^{\lambda} \rho \\
& +\bar{\eta}^{\mathrm{H}}(\delta \wedge \epsilon)^{\mu}{ }_{\nu \rho}^{\lambda}+\bar{\gamma}\left(\delta \wedge \sigma^{x}\right)^{\mu}{ }_{\nu \rho}^{\lambda}+\bar{\Theta}\left(\sigma^{z} \wedge \delta\right)^{\mu}{ }_{\nu}{ }^{\lambda} \rho
\end{aligned}
$$


where $\sigma^{x}$ and $\sigma^{z}$ are Pauli matrices, $\epsilon$ is the twodimensional Levi-Civita symbol, $\delta$ is the Kronecker delta, and $\wedge$ represents the antisymmetrized tensor product. We see that there are six independent Hall viscosity coefficients. First, the three coefficients $\eta^{\mathrm{H}}, \gamma$, and $\Theta$ are traceless on the last pair of indices and so do not involve compression of the fluid. Here, $\eta^{\mathrm{H}}$ is the ordinary isotropic Hall viscosity, while $\gamma$ and $\Theta$ explicitly break threefold and fourfold rotational symmetry and involve the antisymmetric stress. The three "barred" coefficients, $\bar{\eta}^{\mathrm{H}}, \bar{\gamma}$, and $\bar{\Theta}$, have nonvanishing trace. As with the unbarred coefficients, $\bar{\eta}^{\mathrm{H}}$ is rotationally invariant, while $\bar{\gamma}$ and $\bar{\Theta}$ explicitly break threefold and fourfold rotational symmetry. However, although the tensor structure of $\bar{\eta}^{\mathrm{H}}$ is isotropic, it explicitly generates antisymmetric stress. Thus, in a rotationally invariant system, we must have $\bar{\eta}^{\mathrm{H}}=0$ when the symmetric (Belinfante) stress tensor is used, even for a compressible fluid. This requirement is our first indication that a proper examination of anisotropic Hall viscosity involves a careful extension of the Belinfante stress tensor to nonrotationally invariant systems. To our knowledge, the barred Hall viscosities have not been emphasized previously in the quantum mechanics literature (though see Ref. [13]). Consequently, we believe the anomalous antisymmetric stress in Ref. [49] can now be attributed to $\bar{\eta}^{\mathrm{H}}$.

To understand the meaning of these viscosity coefficients, let us return to the continuity equation (2). In the presence of a fluid velocity gradient $\partial_{\mu} v^{\nu}$, the viscosity creates an additional stress

$$
\delta \tau_{\mathrm{B}, \nu}^{\mu}=-\sum_{\lambda \rho} \eta_{\nu}^{\mu \lambda}{ }_{\nu}^{\lambda} \partial_{\lambda} v^{\rho} .
$$

Inserting this stress into the continuity equation gives a viscous contribution to the force density,

$$
f_{\nu}^{\eta}=\sum_{\lambda \rho \mu} \eta^{\mu}{ }_{\nu}^{\lambda} \partial_{\rho} \partial_{\lambda} \partial_{\mu} v^{\rho} .
$$

We see that only the components of the viscosity tensor that are symmetric under the exchange $\mu \rightarrow \lambda$ contribute to the viscous forces. If we focus explicitly on the nondissipative forces

$$
f_{\nu}^{\mathrm{H}, \eta}=\sum_{\lambda \rho \mu}\left(\eta^{\mathrm{H}}\right)_{\nu}^{\mu}{ }_{\nu}^{\lambda} \partial_{\lambda} \partial_{\mu} v^{\rho}
$$

we find, from our decomposition in Eq. (76), that

$$
\begin{aligned}
f_{\nu}^{\mathrm{H}, \eta} & =\sum_{\substack{\mu \nu^{\prime} \rho^{\prime} \\
\rho \lambda}} \frac{1}{2}\left(\epsilon^{\nu^{\prime} \rho^{\prime}}\left(\eta^{\mathrm{H}}\right)^{\mu}{ }_{\nu^{\prime}}{ }^{\prime}{ }^{\prime}\right) \partial_{\mu} \partial_{\lambda}\left(\epsilon_{\nu \rho} v^{\rho}\right) \\
& \equiv \sum_{\mu \lambda \rho} \eta_{\mathrm{H}}^{\mu \lambda} \partial_{\mu} \partial_{\lambda}\left(\epsilon_{\nu \rho} v^{\rho}\right) .
\end{aligned}
$$

In going from the first to the second line, we have used the antisymmetry of the Hall viscosity tensor, as well as the explicit symmetry of Eq. (78) under the interchange of $\mu$ and $\lambda$. This process defines the Hall tensor

$$
\eta_{\mathrm{H}}^{\mu \nu}=\frac{1}{4} \sum_{\lambda \rho} \epsilon^{\lambda \rho}\left(\eta^{\mu}{ }_{\lambda}^{\nu} \rho+\eta^{\nu}{ }_{\lambda}^{\mu} \rho\right)
$$

which coincides with the contracted Hall viscosity introduced in the study of the quantum Hall effect in Refs. [10,50,51]. In those works, the Hall tensor was introduced to parametrize the quadrupole ("internal" or "second" metric) degrees of freedom in anisotropic quantum Hall states; here, we see that this same tensor governs the relationship between velocity gradients and internal forces in the fluid.

As a rank-two symmetric tensor, we see that the Hall tensor has at most three independent components, even in the absence of any symmetries. This feature means that in anisotropic systems, not all odd viscosities result in independent forces in the bulk. As an example, let us consider a (possibly compressible) twofold symmetric fluid. Using Eq. (76), we find, for the Hall tensor,

$$
\eta_{\mathrm{H}}^{\mu \nu}=\left(\eta^{\mathrm{H}}+\bar{\eta}^{\mathrm{H}}\right) \delta^{\mu \nu}+(\gamma+\bar{\gamma}) \sigma_{z}^{\mu \nu}+(\Theta+\bar{\Theta}) \sigma_{x}^{\mu \nu} .
$$

We see that, at the level of forces, the compressive barred Hall viscosities are indistinguishable from the traceless unbarred viscosities. Thus, we should treat both viscosities on equal footing.

Going further, we can shift contributions between the barred and unbarred viscosity coefficients by adding divergenceless terms to the stress tensor. Explicitly, consider a contact term that redefines the stress tensor by

$$
\delta \tau^{\mu}{ }_{\nu}=\sum_{\lambda \rho} \epsilon^{\mu \lambda} C_{\nu \rho} \partial_{\lambda} v^{\rho},
$$

where $C_{\nu \rho}$ is a generic symmetric tensor. Because of the epsilon tensor, $\sum_{\mu} \partial_{\mu} \delta \tau^{\mu}{ }_{\nu}=0$ by construction, so this redefinition of the stress tensor does not change the viscous force density, Eq. (78). At the level of viscosities, however, the contact term in Eq. (84) contributes to the difference between the barred and unbarred viscosity coefficients. Concretely, if we write

$$
C_{\nu \rho}=C_{0} \delta_{\nu \rho}+C_{x} \sigma_{\nu \rho}^{x}+C_{z} \sigma_{\nu \rho}^{z}
$$

then, by contracting the tensor $\epsilon^{\mu \lambda} C_{\nu \rho}$ with the antisymmetric products of matrices in the decomposition Eq. (76), we find that the contact term shifts the viscosities by

$$
\begin{aligned}
\eta^{\mathrm{H}} \rightarrow \eta^{\mathrm{H}}+C_{0}, & \bar{\eta}^{\mathrm{H}} \rightarrow \bar{\eta}^{\mathrm{H}}-C_{0}, \\
\gamma \rightarrow \gamma+C_{z}, & \bar{\gamma} \rightarrow \bar{\gamma}-C_{z}, \\
\Theta \rightarrow \Theta+C_{x}, & \bar{\Theta} \rightarrow \bar{\Theta}-C_{x} .
\end{aligned}
$$


Because we define the stress tensor directly through the continuity equation (2), the value of the tensor $C_{\nu \rho}$ is a priori undetermined. While in field theories there may be underlying principles for coupling particles to geometry that fix the divergence-free contact terms, condensed matter models-especially those derived from lattice modelslack such guiding principles. As such, the sum of the barred and unbarred viscosities appearing in Eq. (83) give precisely the responses that can be calculated and physically probed in a model-independent fashion. It is only in the presence of dynamical gravity sourced by the energymomentum tensor that the effect of $C_{\mu \nu}$ can be observed in the bulk. In a fluid with boundaries, however, these redundant terms will result in different contributions to the tangent and normal forces on the surface. In this case, a general principle regarding the choice of boundary conditions is still needed to disambiguate barred and unbarred contributions to the viscosity tensor.

It is also important to note that the contact term (84) can be nonzero even for incompressible flows satisfying $\nabla \cdot \mathbf{v}=0$. Thus, we should expect both the barred and unbarred viscosities to be nonzero even in incompressible anisotropic systems.

Finally, while we do not focus on the dissipative viscosity

$$
\left(\eta^{\mathrm{D}}\right)^{\mu}{ }_{\nu \rho}^{\lambda} \equiv \frac{1}{2}\left(\eta^{\mu}{ }_{\nu}{ }^{\lambda}{ }_{\rho}+\eta^{\lambda}{ }_{\rho}^{\mu}{ }_{\nu}\right)
$$

in this work, it is curious to note that there exists a similar ambiguity there as well. In particular, choosing the tensor $C_{\mu \nu}$ to be antisymmetric gives a dissipative viscous stress that does not appear as a viscous force. Thus, of the ten possible independent dissipative viscosities without symmetry, only nine appear in the continuity equation. The physical implications of this result for hydrodynamics and entropy production will be a focus of future work.

\section{Symmetry constraints on the viscosity tensor}

Finally, let us analyze the symmetries of the viscosity coefficients in Eq. (76) in more detail. We analyze how point group symmetries constrain the decomposition of the Hall viscosity (76). A useful point of view for this aim is to regard the two-tensors $\sigma^{x}, \sigma^{z}, \epsilon$, and $\delta$ as Clebsch-Gordan coefficients: Each tensor is a map that takes two vectors as input and returns an element in a representation of the point group. For instance, the Kronecker delta $\delta_{\nu}^{\mu}$ is a map that takes a vector $v^{\nu}$ and a dual vector $u_{\mu}$ as inputs and returns an object $v^{\mu} u_{\mu}$, which is a scalar under all point group symmetries. In order for the viscosity tensor to respect the point group symmetries of the system, we demand that the fully contracted object

$$
\sum_{\mu \nu \lambda \rho}\left(\eta^{\mathrm{H}}\right)_{\nu}^{\mu}{ }_{\nu \rho}^{\lambda} a_{\mu} b^{\nu} c_{\lambda} d^{\rho}
$$

transform in the trivial (scalar) representation of the point group. Applying this logic, we then must ask which of the terms in Eq. (76) project onto the trivial representation. Note that for lattice systems with threefold rotational symmetry, there is an additional subtlety in identifying the proper Clebsch-Gordan coefficients because the indices on the stress tensor and momentum density do not transform tensorially under threefold rotational symmetry. As this case is not relevant for the examples treated here, we will defer the systematic treatment to future work.

Let us focus first on rotational symmetry. Under the action of the continuous rotational symmetry group, the tensors $\delta_{\nu}^{\mu}$ and $\epsilon^{\mu}{ }_{\nu}$ are both maps to the scalar representation. On the other hand, the two Pauli matrices $\left(\sigma^{x}, \sigma^{z}\right)$ transform as a two-dimensional quadrupole representation

$$
\left(\begin{array}{c}
\sigma_{x} \\
\sigma_{z}
\end{array}\right) \rightarrow\left(\begin{array}{cc}
\cos 2 \theta & \sin 2 \theta \\
-\sin 2 \theta & \cos 2 \theta
\end{array}\right)\left(\begin{array}{c}
\sigma_{x} \\
\sigma_{z}
\end{array}\right)
$$

under a rotation by angle $\theta$. Since both the product of two scalars and the antisymmetric product of two vectors are again scalars, we deduce that with continuous $S O(2)$ rotational symmetry, only $\eta^{\mathrm{H}}$ and $\bar{\eta}^{\mathrm{H}}$ can be nonzero. Our Belinfante construction additionally ensures that $\bar{\eta}^{\mathrm{H}}=0$ in this case, as we can deduce from the symmetry of $T_{\mathrm{B}, \nu}^{\mu}$ entering the Kubo formula, Eq. (70).

Furthermore, for any discrete rotational symmetry group including a rotation of order 3 or greater (except the group $C_{4}$ generated by a fourfold rotation), the quadrupole representation remains an irreducible representation. For the group $C_{4}$, the quadrupole representation reduces to two copies of the sign representation $\Delta$, with $\Delta\left(C_{4}\right)=-1$ [52-54]. In both cases, we find that the only scalar viscosities are $\eta^{\mathrm{H}}$ and $\bar{\eta}^{\mathrm{H}}$, with all other coefficients vanishing identically. Unlike with continuous rotational symmetry, with discrete symmetry we must allow $\bar{\eta}^{\mathrm{H}} \neq 0$.

In addition to the discrete rotational symmetries, we can also think about the action of parity $P$ and time reversal $T$ on the viscosity tensor, as well as the composite symmetry $P T$. We see that parity and $P T$, in particular, trim down the number of nonzero components of the Hall viscosity tensor [55]. By Onsager reciprocity [56], we know that the Hall viscosity is odd under time-reversal symmetry,

$$
T:\left(\eta^{\mathrm{H}}\right)^{\mu}{ }_{\nu}{ }^{\lambda} \rho-\left(\eta^{\mathrm{H}}\right)_{\nu}^{\mu}{ }_{\nu}{ }^{\lambda} .
$$

Let us now consider the effects of parity symmetry. In two spatial dimensions, parity coincides with a crystallographic mirror symmetry and hence requires a choice of axis. For concreteness, let us, for now, take $P$ to be the mirror symmetry $M_{x}$, 


$$
P \equiv M_{x}: x \rightarrow-x, \quad y \rightarrow y .
$$

Under the action of this transformation,

$$
\begin{aligned}
\delta & \rightarrow P^{T} \delta P=\delta, \\
\epsilon & \rightarrow P^{T} \epsilon P=-\epsilon, \\
\sigma_{x} & \rightarrow P^{T} \sigma_{x} P=-\sigma_{x}, \\
\sigma_{z} & \rightarrow P^{T} \sigma_{z} P=\sigma_{z} .
\end{aligned}
$$

The diagonal Clebsch-Gordan coefficients $\delta, \sigma^{z}$ are parity even, while the off-diagonal ones $\epsilon, \sigma^{x}$ are parity odd. When combined with the decomposition (76), under parity symmetry, $\eta^{\mathrm{H}}, \bar{\eta}^{\mathrm{H}}, \gamma$, and $\bar{\gamma}$ are odd, while $\Theta$ and $\bar{\Theta}$ are even. Thus, in a $P$-symmetric system, only $\Theta$ and $\bar{\Theta}$ can be nonzero.

Furthermore, since all Hall viscosity coefficients are $T$ odd, we can easily deduce the effect of the composite symmetry $P T$ : It flips the sign of $\Theta$ and $\bar{\Theta}$ while leaving the other Hall viscosities invariant. Thus, in systems with $P T$ symmetry but neither $P$ or $T$ individually, there are only four possible nonzero viscosity coefficients, $\eta^{\mathrm{H}}, \bar{\eta}^{\mathrm{H}}, \gamma$, and $\bar{\gamma}$. This case is consistent with the symmetry analysis of Refs. [10,55]. We often work with $P T$-invariant systems, where we can say, in general, that $\Theta=\bar{\Theta}=0$.

The analysis so far was done for parity symmetry defined to be the mirror reflection $M_{x}$. For a more general reflection axis, it is still true that four viscosity coefficients are parity odd, and two viscosity coefficients are parity even. More concretely, let us consider the reflection $M_{R x}$ defined as

$$
M_{R x}=R(\theta) M_{x} R^{T}(\theta),
$$

in terms of the rotation matrix $R(\theta)$ in Eq. (15). Under this reflection, the Clebsch-Gordan tensor $\delta$ is invariant, $\epsilon$ is odd, and the coefficients $\left(\sigma^{x}, \sigma^{z}\right)$ transform as

$$
\left(\begin{array}{c}
\sigma^{x} \\
\sigma^{z}
\end{array}\right) \rightarrow\left(\begin{array}{cc}
-\cos 2 \theta & -\sin 2 \theta \\
-\sin 2 \theta & \cos 2 \theta
\end{array}\right)\left(\begin{array}{l}
\sigma^{x} \\
\sigma^{z}
\end{array}\right)
$$

meaning that the linear combination $\cos \theta \sigma^{z}-\sin \theta \sigma^{x}$ is invariant under $M_{R x}$, while the linear combination $\cos \theta \sigma^{x}+$ $\sin \theta \sigma^{z}$ is odd under $M_{R x}$. It follows then that $\eta^{\mathrm{H}}, \bar{\eta}^{\mathrm{H}}$, as well as

$$
\begin{aligned}
& \gamma_{R} \equiv \gamma \cos \theta-\Theta \sin \theta, \\
& \bar{\gamma}_{R} \equiv \bar{\gamma} \cos \theta+\bar{\Theta} \sin \theta,
\end{aligned}
$$

are odd under $M_{R x}$, while the orthogonal linear combinations

$$
\begin{gathered}
\Theta_{R} \equiv \Theta \cos \theta+\gamma \sin \theta, \\
\bar{\Theta}_{R} \equiv \bar{\Theta} \cos \theta-\bar{\gamma} \sin \theta
\end{gathered}
$$

are even under $M_{R x}$. Our previous results on $P T$ symmetry thus readily generalize by replacing $\gamma, \bar{\gamma}, \Theta$, and $\bar{\Theta}$ by their transformed counterparts (97)-(100), respectively.

We are now able to begin a discussion on the viscosity of lattice and continuum systems, keeping the constraints provided by rotational and $P T$ symmetries in mind. When dealing with systems with internal degrees of freedom, such as spin or pseudospin, it will be important to establish the representation of rotations and reflections in order to ultimately verify the symmetries of the viscosity tensor.

\section{Viscosity-conductivity relation and other tensors}

We note that the viscosity-conductivity relation [Eq. (4.10) in Ref. [37]] links the finite wave-vector conductivity to the stress response $X$, which is related to the viscosity, as (in the case of zero external magnetic field)

$$
\frac{\partial^{2} \sigma_{\nu \rho}}{\partial q_{\mu} \partial q_{\lambda}} \propto X_{\mu \nu \lambda \rho}+X_{\lambda \nu \mu \rho}
$$

Therefore, the $(q=0)$ Hall viscosity has a direct connection to the $q^{2}$ part of the conductivity tensor. Note that the symmetry of Eq. (101) under the exchange of $\mu \leftrightarrow \lambda$ implies that only the Hall tensor (i.e., only the combinations of viscosity coefficients that contribute to forces) enters into the conductivity. This result holds generally for Galilean-invariant systems with or without rotational symmetry. Hence, this relation holds for the anisotropic systems we consider, provided the viscosity response is expanded to include the novel coefficients in Eq. (76). Going to higher orders in momentum, one can consider the link between the full finite wave-vector viscosity and conductivity, noting that at nonzero momentum, the transformation of the viscosity tensor under $P T$ symmetry changes accordingly [55].

Regarding rotational symmetry, we note that through the viscosity-conductivity relation, the viscosity is connected to another fourth rank tensor, which gives the stress response to electric field gradients. The same component-by-component symmetry analysis we consider can be applied here (and, in fact, to any response function), with the caveat that properties under time-reversal symmetry are not the same as those of the viscosity tensor (electric field gradients and strain rates do not have the same behavior under time reversal). It would be interesting to connect irreducible representations of the viscosity tensor to those of the electric field gradient response tensor using the viscosity-conductivity relation as a mapping.

As a last point, we mention that the fourth rank tensor that shares the most properties with the viscosity is the tensor of elastic moduli $\kappa$. For example, for the Hall elastic modulus $[12,57]\left(\kappa^{\mathrm{H}}\right)^{\mu}{ }_{\nu}{ }^{\lambda} \rho$, the force redundancy statement in Eq. (78) also holds. Similar to the Hall viscosity, the Hall elastic modulus is antisymmetric under $(\mu \nu) \leftrightarrow(\lambda \rho)$ 
(except, unlike the viscosity, it is only nonvanishing in driven systems).

\section{FREE FERMIONS: HALL VISCOSITY AND BERRY CURVATURE}

Using this general formalism, we can now examine the Hall viscosity in anisotropic free-fermion systems, both in the continuum and on the lattice. We take as our unperturbed Hamiltonian

$$
H_{0}=\sum_{n m \mathbf{k}} c_{n \mathbf{k}}^{\dagger} f^{n m}(\mathbf{k}) c_{m \mathbf{k}} \cdot
$$

Below, we first consider the stress response in anisotropic continuum systems, where $f^{n m}(\mathbf{k})$ can be any smooth function of $\mathbf{k}$. Second, we consider lattice systems, where $f^{n m}(\mathbf{k})$ satisfies the additional reciprocal lattice periodicity constraints. In both formalisms, we see how the Hall viscosities simultaneously capture the effects of Berry curvature and internal angular momentum.

\section{A. Continuum viscosity}

For a continuum free-fermion system with Hamiltonian (102), we can compute the stress tensor explicitly from the form of the strain generator (21) and the definition (22) to find

$$
\begin{aligned}
T_{\mathrm{B}, \nu}^{\mu} & =-i\left[H_{0}, \mathcal{J}^{\mu}{ }_{\nu}\right] \\
& =\sum_{n m \mathbf{k}} c_{n \mathbf{k}}^{\dagger}\left(k_{\nu} \partial^{\mu} f^{n m}(\mathbf{k})+\frac{i}{2} \epsilon^{\mu}{ }_{\nu}\left[f(\mathbf{k}), L_{\mathrm{int}}\right]_{n m}\right) c_{m \mathbf{k}}
\end{aligned}
$$

\section{Equilibrium stress}

Before inserting this expression into the Kubo formula (70), it is worth looking at some general properties of the free-fermion stress. First, let us reiterate that for a rotationally invariant system,

$$
i\left[f(\mathbf{k}), L_{\text {int }}\right]_{n m} \rightarrow k_{x} \partial^{y} f_{n m}(\mathbf{k})-k_{y} \partial^{x} f_{n m}(\mathbf{k}),
$$

guaranteeing that the stress tensor will be symmetric in this case. Next, let us look at the equilibrium stress $\left\langle T_{\mathrm{B}, \nu}^{\mu}\right\rangle_{0}$ in a zero-temperature ground state with chemical potential $E_{F}$. We introduce the diagonalizing unitary transformation

$$
f^{n m}(\mathbf{k})=\sum_{\alpha} U_{n \alpha}^{\dagger}(\mathbf{k}) U_{\alpha m}(\mathbf{k}) \epsilon_{\alpha}(\mathbf{k}),
$$

where $\epsilon_{\alpha}(\mathbf{k})$ are the single-particle eigenvalues of $H_{0}$. This transformation allows us to write the single-particle Green's function as
$G_{n m}(\mathbf{k})=\left\langle c_{n \mathbf{k}}^{\dagger} c_{m \mathbf{k}}\right\rangle_{0}=\sum_{\alpha} n_{F}\left[\epsilon_{\alpha}(\mathbf{k})\right] U_{m \alpha}^{\dagger}(\mathbf{k}) U_{\alpha n}(\mathbf{k})$,

where $n_{F}$ is the Fermi distribution function. Taking the ground-state average of Eq. (103) and using Eq. (106) yields, after some simplification,

$$
\left\langle T_{\mathrm{B}, \nu}^{\mu}\right\rangle_{0}=\sum_{\alpha \mathbf{k}} n_{F}\left[\epsilon_{\alpha}(\mathbf{k})\right] k_{\nu} \partial^{\mu} \epsilon_{\alpha}(\mathbf{k}) .
$$

Importantly, the spin stress does not contribute to the ground-state average since the average of a commutator of (finite rank) operators vanishes. Using this expression, we can prove an important theorem about the ground-state stress in a continuum free-fermion system,

$$
\left\langle T_{\mathrm{B}, \nu}^{\mu}\right\rangle_{0}=\frac{1}{2} \delta_{\nu}^{\mu}\left\langle\operatorname{tr}\left(T_{\mathrm{B}}\right)\right\rangle_{0} .
$$

In other words, the average stress of a free-fermion system is isotropic. To prove this, let us first rewrite Eq. (107) as an integral over momentum space,

$$
\left\langle T_{\mathrm{B}, \nu}^{\mu}\right\rangle_{0}=\frac{V}{4 \pi^{2}} \int d^{2} k n_{F}\left(\epsilon_{\alpha}\right) k_{\nu} v_{\alpha}^{\mu},
$$

where $v_{\alpha}^{\mu} \equiv \partial^{\mu} \epsilon_{\alpha}$ is the group velocity of band $\alpha$. To simplify this further, let us do a coordinate transformation to a basis of constant-energy contours. Let us write

$$
\mathbf{k}_{a \alpha}=k_{a \alpha}(\theta, \epsilon)(\cos \theta, \sin \theta),
$$

where the new index $a$ runs over possible disjoint branches of the inverse of $\epsilon_{\alpha}(\mathbf{k})$. Note that care must be taken due to the inverse function, and we cannot assume that partial derivatives with respect to $\theta$ and $\epsilon$ commute. Since the number of values of the index $a$ may depend on $\epsilon$, we must also take care to integrate over $\theta$ and sum over $a$ before integrating over $\epsilon$. With these caveats in mind, Eq. (109) becomes

$\left\langle T_{\mathrm{B}, \nu}^{\mu}\right\rangle_{0}=\frac{V}{4 \pi^{2}} \int_{0}^{\infty} d \epsilon n_{F}(\epsilon) \sum_{\alpha a} \int_{0}^{2 \pi} d \theta\left|J_{a \alpha}\right| k_{a \alpha \nu} v_{a \alpha}^{\mu}$,

where $\left|J_{a \alpha}\right|$ is the Jacobian determinant for the transformation, given by

$$
\left|J_{a \alpha}\right|=k_{a \alpha} \partial_{\epsilon} k_{a \alpha} .
$$

Additionally, the inverse of the Jacobian matrix yields the velocity

$$
v_{a \alpha}^{\mu}=\sum_{\nu} \epsilon^{\mu \nu} \frac{\partial_{\theta} k_{a \alpha \nu}}{\left|J_{a \alpha}\right|} .
$$


Putting these pieces together gives

$$
\left\langle T_{\mathrm{B}, \nu}^{\mu}\right\rangle 0=\frac{V}{4 \pi^{2}} \int_{0}^{\infty} d \epsilon n_{F}(\epsilon) \sum_{a \alpha \lambda} \int_{0}^{2 \pi} d \theta \epsilon^{\mu \lambda} k_{a \alpha \nu} \partial_{\theta} k_{a \alpha \lambda} .
$$

When contracted with $\epsilon_{\mu}{ }^{\nu}, \sigma_{\mu}^{x, \nu}$, and $\sigma_{\mu}^{z, \nu}$, the integrand in Eq. (114) can be rewritten as a total $\theta$ derivative, and hence the integral vanishes. On the other hand, when contracted with $\delta_{\nu}^{\mu}$, the integrand in Eq. (114) becomes the differential area element $\mathbf{k}_{a \alpha} \times \partial_{\theta} \mathbf{k}_{a \alpha} d \theta$ enclosed by the constant energy surfaces in Eq. (110). We thus conclude that

$$
\left\langle T_{\mathrm{B}, \nu}^{\mu}\right\rangle_{0}=\frac{V}{8 \pi^{2}} \delta_{\nu}^{\mu} \int_{0}^{\infty} d \epsilon n_{F}(\epsilon) A(\epsilon),
$$

where $A(\epsilon)$ is the momentum-space area enclosed by the constant- $\epsilon$ contour. Note that this result holds at any temperature and even in the absence of rotational symmetry. We thus see that equilibrium stress in an anisotropic Fermi gas is isotropic. We now make use of this result to simplify the contact term in the nondissipative stress response.

\section{Stress response}

We examine the Hall stress response (72). First, let us focus on the contact term in the Kubo formula, which gives the "Hall elastic modulus" [12,57]. Starting from Sec. (IVA) and focusing on the antisymmetric contribution to the contact term,

$$
C_{\nu \rho}^{\mu \lambda}=\frac{i}{2 \omega}\left(\left\langle\left[T_{\mathrm{B}, \nu}^{\mu}, \mathcal{J}_{\rho}^{\lambda}\right]\right\rangle_{0}-\left\langle\left[T_{\mathrm{B}, \rho}^{\lambda}, \mathcal{J}^{\mu}{ }_{\nu}\right]\right\rangle_{0}\right),
$$

we can use the Jacobi identity and the Ward identity (22) to find

$$
\begin{aligned}
C_{\nu \rho}^{\mu \lambda} & =\frac{1}{2 \omega}\left(\left\langle\left[\left[H_{0}, \mathcal{J}^{\mu}{ }_{\nu}\right], \mathcal{J}^{\lambda}{ }_{\rho}\right]\right\rangle_{0}-\left\langle\left[\left[H_{0}, \mathcal{J}^{\lambda}{ }_{\rho}\right], \mathcal{J}^{\mu}{ }_{\nu}\right]\right\rangle_{0}\right) \\
& =\frac{1}{2 \omega}\left(\left\langle\left[\left[H_{0}, \mathcal{J}^{\mu}{ }_{\nu}\right], \mathcal{J}^{\lambda}{ }_{\rho}\right]\right\rangle_{0}+\left\langle\left[\left[\mathcal{J}_{\rho}^{\lambda}, H_{0}\right], \mathcal{J}^{\mu}{ }_{\nu}\right]\right\rangle_{0}\right) \\
& =-\frac{1}{2 \omega}\left\langle\left[\left[\mathcal{J}^{\mu}{ }_{\nu}, \mathcal{J}^{\lambda}{ }_{\rho}\right], H_{0}\right]\right\rangle_{0} .
\end{aligned}
$$

The commutator of strain generators can then be simplified using the algebra (30). We find, using the kinetic Ward identity (12),

$$
\begin{aligned}
C_{\nu \rho}^{\mu \lambda} & =\frac{i}{2 \omega}\left(\left\langle\left[J_{\nu}^{\lambda}, H_{0}\right]\right\rangle_{0} \delta_{\rho}^{\mu}-\left\langle\left[J_{\rho}^{\mu}, H_{0}\right]\right\rangle_{0} \delta_{\mu}^{\lambda}\right) \\
& =\frac{1}{2 \omega}\left(\left\langle T^{\lambda}{ }_{\nu}\right\rangle_{0} \delta_{\rho}^{\mu}-\left\langle T^{\mu}{ }_{\rho}\right\rangle_{0} \delta_{\nu}^{\lambda}\right) .
\end{aligned}
$$

Finally, using the results of Eq. (107),

$$
\left\langle T^{\mu}{ }_{\nu}\right\rangle_{0}=\left\langle T_{\mathrm{B}, \nu}^{\mu}\right\rangle_{0} \propto \delta_{\nu}^{\mu},
$$

we see that

$$
C^{\mu}{ }_{\nu \rho}^{\lambda}=0 .
$$

Thus, the anisotropic free Fermi gas does not have a Hall elastic modulus. This case is consistent with the interpretation of the continuum free Fermi gas as a fluid and should be contrasted with the results of Ref. [12] for a quasi-twodimensional system.

Finally, we can compute the Hall viscosity of the continuum free Fermi gas,

$$
\begin{aligned}
\left(\eta^{\mathrm{H}}\right)_{\nu}^{\mu}{ }_{\nu} \lambda= & \frac{1}{2 \omega^{+} V} \int_{0}^{\infty} d t e^{i \omega^{+} t}\left(\left\langle\left[T_{\mathrm{B}, \nu}^{\mu}(t), T_{\mathrm{B}, \rho}^{\lambda}(0)\right]\right.\right. \\
& \left.\left.-\left[T_{\mathrm{B}, \rho}^{\lambda}(t), T_{\mathrm{B}, \nu}^{\mu}(0)\right]\right\rangle\right) .
\end{aligned}
$$

Since all the operators in the Kubo formula (70) are singleparticle operators, we can evaluate the viscosity using the single-particle Green's function (106). It is convenient to evaluate separately the two contributions

$$
\left(\eta^{\mathrm{H}}\right)_{\nu \rho}^{\mu}{ }_{\nu}^{\lambda}=\left(\eta_{\mathrm{kin}}^{\mathrm{H}}\right)^{\mu}{ }_{\nu \rho}^{\lambda}+\left(\eta_{\mathrm{int}}^{\mathrm{H}}\right)_{\nu}^{\mu}{ }_{\rho}^{\lambda},
$$

where we define the kinetic viscosity $\eta_{\text {kin }}^{\mathrm{H}}$ to be the time integral evaluated with the kinetic (or strain) stress tensor $T^{\mu}{ }_{\nu}$ only, and $\eta_{\text {int }}^{\mathrm{H}}$ to be the contributions to the viscosity from the internal angular momentum. Because the spin stress in Eq. (103) is purely antisymmetric, it does not contribute to the symmetric viscosity coefficients $\eta^{\mathrm{H}}, \bar{\gamma}$, or $\bar{\Theta}$, which can be extracted entirely from the kinetic contributions. Evaluating the kinetic contribution first, we can insert a complete set of states and use the eigenbasis (105) to carry out the time integral. We find that

$$
\begin{aligned}
\left(\eta_{\text {kin }}^{\mathrm{H}}\right)_{\nu}^{\mu}{ }_{\nu \rho}^{\lambda}= & \frac{1}{4 \pi^{2}} \int d^{2} k \sum_{n m \alpha \beta} \frac{\left[\epsilon_{\alpha}-\epsilon_{\beta}\right)^{2}\left(n\left(\epsilon_{\beta}\right)-n\left(\epsilon_{\alpha}\right)\right]}{\omega^{2}+\left(\epsilon_{\alpha}-\epsilon_{\beta}\right)^{2}} \\
& \times k_{\nu}\left(\partial^{\mu} U_{\beta n}\right) U_{n \alpha}^{\dagger} k_{\rho}\left(\partial^{\lambda} U_{\alpha m}\right) U_{m \beta}^{\dagger} .
\end{aligned}
$$

In the limit of zero frequency and zero temperature, this expression simplifies considerably. Recalling that the Berry connection $\mathbf{A}_{\alpha \beta}$ is given by

$$
\left(\partial_{\nu} U_{\beta n}\right) U_{n \alpha}^{\dagger}=\left\langle\partial^{\nu} u_{\beta \mathbf{k}} \mid u_{\alpha \mathbf{k}}\right\rangle \equiv i A_{\beta \alpha}^{\nu},
$$

in terms of the single-particle eigenstates $\left|u_{\alpha \mathbf{k}}\right\rangle$, we find that at zero temperature

$$
\left(\eta_{\text {kin }}^{\mathrm{H}}\right)_{\nu \rho}^{\mu{ }_{\nu}{ }^{2}}=\frac{1}{4 \pi^{2}} \int_{\mathrm{occ}} d^{2} k k_{\nu} k_{\rho} \epsilon^{\mu \lambda} \operatorname{tr}(\Omega),
$$

where $\Omega$ is the non-Abelian Berry curvature viewed as a matrix in the space of occupied states at $\mathbf{k}$ [58], and $\int_{\text {occ }}$ 
denotes an integral over occupied states. Recalling Eq. (76) and noting again that $\left(\eta_{\mathrm{kin}}^{\mathrm{H}}\right)_{\nu}^{\mu}{ }_{\rho}{ }_{\rho}$ is the only contribution to the symmetric Hall viscosity coefficients, we find that

$$
\begin{gathered}
\eta^{\mathrm{H}}=\frac{1}{16 \pi^{2}} \int_{\mathrm{occ}} d^{2} k\left(k_{x}^{2}+k_{y}^{2}\right) \operatorname{tr}(\Omega), \\
\bar{\gamma}=\frac{1}{16 \pi^{2}} \int_{\mathrm{occ}} d^{2} k\left(k_{x}^{2}-k_{y}^{2}\right) \operatorname{tr}(\Omega), \\
\bar{\Theta}=\frac{1}{8 \pi^{2}} \int_{\mathrm{occ}} d^{2} k k_{x} k_{y} \operatorname{tr}(\Omega) .
\end{gathered}
$$

We see that the coefficients $\eta^{\mathrm{H}}, \bar{\gamma}$, and $\bar{\Theta}$ are given by the quadrupole moments of the Berry curvature, which is one of the main results of this work. Note that since the Berry curvature $\operatorname{tr}(\Omega)$ is $P T$ even, we explicitly see here that $\bar{\Theta}$ is $P T$ odd, while $\eta^{\mathrm{H}}$ and $\bar{\gamma}$ are $P T$ even, consistent with the results of Sec. IV C.

However, note that our expression (125) is antisymmetric on the indices $\mu$ and $\lambda$. From our discussion in Eq. (84), we see that this is precisely the tensor structure for a term that does not contribute to the equations of motion. In other words, we see from Eq. (78) for the viscous forces that the antisymmetric viscosities $\bar{\eta}^{\mathrm{H}}, \gamma$, and $\Theta$ computed from Eq. (125) exactly cancel the viscous forces due to the symmetric viscosities (126)-(128), which leads us to our second main result, namely, that the continuum Hall tensor is entirely determined from the internal angular-momentum contribution to the stress. This result holds true even at finite frequency and temperature, due to the antisymmetry of Eq. (123) under the exchange of $\mu \leftrightarrow \lambda$.

At zero frequency and temperature, we can evaluate the remainder of the Kubo formula in a simplified form. Using the same spectral decomposition leading to Eq. (123), we find, in the limit of zero frequency and zero temperature, that

$$
\begin{aligned}
\left(\eta_{\text {int }}^{\mathrm{H}}\right)_{\nu}^{\mu \lambda}= & \sum_{\alpha \beta} \frac{1}{4 \pi^{2}} \int d^{2} k\left[n\left(\epsilon_{\alpha}\right)-n\left(\epsilon_{\beta}\right)\right] L_{\text {int }}^{\alpha \beta} \\
& \times\left(\epsilon_{\rho}^{\lambda} k_{\nu} A_{\beta \alpha}^{\mu}-\epsilon^{\mu}{ }_{\nu} k_{\rho} A_{\beta \alpha}^{\lambda}\right),
\end{aligned}
$$

where we have introduced

$$
L_{\text {int }}^{\alpha \beta}=\left\langle u_{\alpha \mathbf{k}}\left|L_{\text {int }}\right| u_{\beta \mathbf{k}}\right\rangle=U_{\alpha n} L_{\text {int }}^{n m} U_{m \beta}^{\dagger}
$$

as the matrix elements of the internal angular momentum in the basis of single-particle energy eigenstates $\left|u_{\alpha \mathbf{k}}\right\rangle$. To see that this expression is gauge invariant, we can rewrite it in terms of projectors

$$
P(\mathbf{k})=\sum_{\mathbf{k} \alpha} n_{F}\left(\epsilon_{\alpha}\right)\left|u_{\alpha \mathbf{k}}\right\rangle\left\langle u_{\alpha \mathbf{k}}\right|
$$

onto the set of occupied bands to find

$$
\begin{aligned}
\left(\eta_{\mathrm{int}}^{\mathrm{H}}\right)^{\mu}{ }_{\nu \rho}^{\lambda}= & \frac{1}{2 \pi^{2}} \operatorname{Re} \sum_{\alpha} \int d^{2} k n_{F}\left(\epsilon_{\alpha}\right) \\
& \times\left\{k_{\nu} \epsilon_{\rho}^{\lambda}\left\langle u_{\alpha \mathbf{k}}|L[1-P(\mathbf{k})]| \partial^{\mu} u_{\alpha \mathbf{k}}\right\rangle_{0}\right. \\
& \left.-k_{\rho} \epsilon^{\mu}{ }_{\nu}\left\langle u_{\alpha \mathbf{k}}|L[1-P(\mathbf{k})]| \partial^{\lambda} u_{\alpha \mathbf{k}}\right\rangle_{0}\right\} .
\end{aligned}
$$

In this form, invariance under unitary transformations in the space of occupied bands is manifest due to the factor of $1-P(\mathbf{k})$ that annihilates the inhomogeneous part of the transformation of $\left|\partial^{\mu} u_{\alpha \mathbf{k}}\right\rangle$.

By applying the contractions introduced in Eq. (75) and recombining the kinetic and internal angular-momentum contributions to the viscosity, we arrive at the following relations between the barred and unbarred viscosity coefficients:

$\bar{\eta}^{H}=\sum_{\alpha \beta} \frac{1}{16 \pi^{2}} \int d^{2} k\left[n\left(\epsilon_{\alpha}\right)-n\left(\epsilon_{\beta}\right)\right] L_{\mathrm{int}}^{\alpha \beta} \mathbf{k} \cdot \mathbf{A}_{\beta \alpha}-\eta^{\mathrm{H}}$,

$\gamma=\sum_{\alpha \beta} \frac{1}{16 \pi^{2}} \int d^{2} k\left[n\left(\epsilon_{\alpha}\right)-n\left(\epsilon_{\beta}\right)\right] L_{\mathrm{int}}^{\alpha \beta} \mathbf{k} \cdot \sigma_{z} \cdot \mathbf{A}_{\beta \alpha}-\bar{\gamma}$,

$\Theta=\sum_{\alpha \beta} \frac{1}{16 \pi^{2}} \int d^{2} k\left[n\left(\epsilon_{\alpha}\right)-n\left(\epsilon_{\beta}\right)\right] L_{\mathrm{int}}^{\alpha \beta} \mathbf{k} \cdot \sigma_{x} \cdot \mathbf{A}_{\beta \alpha}-\bar{\Theta}$.

We see then that the effect of the internal angularmomentum contribution to the stress tensor is, for free fermions, to shift the values of the barred viscosity coefficients relative to their unbarred counterparts. In a rotationally invariant system, the fact that $\bar{\eta}^{\mathrm{H}}=\gamma=\bar{\gamma}=$ $\Theta=\bar{\Theta}=0$ implies

$$
\begin{gathered}
\sum_{\alpha \beta} \int d^{2} k\left[n\left(\epsilon_{\beta}\right)-n\left(\epsilon_{\alpha}\right)\right] L_{\mathrm{int}}^{\alpha \beta} \mathbf{k} \cdot \mathbf{A}_{\beta \alpha} \\
\rightarrow \int_{\mathrm{occ}} d^{2} \mathbf{k}\left(k_{x}^{2}+k_{y}^{2}\right) \operatorname{tr}(\Omega), \\
\sum_{\alpha \beta} \int d^{2} k\left[n\left(\epsilon_{\beta}\right)-n\left(\epsilon_{\alpha}\right)\right] L_{\mathrm{int}}^{\alpha \beta} \mathbf{k} \cdot \sigma_{z} \cdot \mathbf{A}_{\beta \alpha} \rightarrow 0, \\
\sum_{\alpha \beta} \int d^{2} k\left[n\left(\epsilon_{\beta}\right)-n\left(\epsilon_{\alpha}\right)\right] L_{\mathrm{int}}^{\alpha \beta} \mathbf{k} \cdot \sigma_{x} \cdot \mathbf{A}_{\beta \alpha} \rightarrow 0 .
\end{gathered}
$$

Before discussing the analogous expressions in the lattice formalism, let us attempt to recast Eqs. (126)-(128) and (133)-(135) in terms of Fermi-surface integrals, in the spirit of Haldane's approach to the anomalous Hall conductance [15]. Let us assume, for simplicity of illustration, that we have a rotationally invariant system with a single occupied 
band. In this case, the Berry curvature is Abelian, and only the isotropic Hall viscosity $\eta^{\mathrm{H}}$ is nonzero. Inserting

$$
\operatorname{tr}(\Omega)=\sum_{\mu \nu} \epsilon_{\mu \nu} \partial^{\mu} A^{\nu}
$$

into the expression (126) and integrating by parts yields

$$
\begin{aligned}
\eta^{\mathrm{H}}= & \sum_{\mu \nu} \frac{1}{16 \pi} \epsilon_{\mu \nu} \int d^{2} k n_{F}(\mathbf{k})|\mathbf{k}|^{2} \partial^{\mu} A^{\nu} \\
= & -\sum_{\mu \nu} \frac{1}{16 \pi} \epsilon_{\mu \nu} \int d^{2} k \partial^{\mu}\left(n_{F}(\mathbf{k})|\mathbf{k}|^{2}\right) A^{\nu} \\
= & \sum_{\mu \nu} \frac{1}{16 \pi} \epsilon_{\mu \nu} \int d^{2} k \delta\left(\epsilon(\mathbf{k})-\epsilon_{F}\right) k_{F}^{2} v_{\mathrm{F}}^{\mu} A^{\nu} \\
& -\sum_{\mu \nu} \frac{1}{8 \pi} \int_{\mathrm{occ}} d^{2} k \epsilon^{\mu}{ }_{\nu} k_{\mu} A^{\nu} \\
= & \frac{k_{F}^{2}}{16 \pi} \int_{\mathrm{FS}} d \mathbf{k}_{F} \cdot \mathbf{A}-\frac{1}{8 \pi} \int_{\mathrm{occ}} d^{2} k \mathbf{k} \times \mathbf{A} \\
= & \frac{k_{F}^{2}}{8} \sigma^{A H E}-\frac{1}{8 \pi} \int_{\mathrm{occ}} d^{2} k \mathbf{k} \times \mathbf{A},
\end{aligned}
$$

where $\sigma^{A H E}$ is Haldane's expression for the Fermi-surface contribution to the anomalous Hall conductivity. Note that rotational symmetry was essential in deriving this expression since only for rotationally invariant systems is the Fermi momentum independent of position along the Fermi surface. In addition to the Fermi-surface contribution, however, we are left with an additional bulk contribution given by the second term. For rotationally invariant systems, each of these contributions is separately gauge invariant $(\bmod 2 \pi)$, and neither is zero, in general.

We can see here that the obstacle to obtaining a purely Fermi-surface expression for the Hall viscosity arises from the explicit factors of $\mathbf{k}$ in the integrands (126)-(128). Once we consider a fully anisotropic system, the situation becomes even worse: The Fermi-surface and bulk terms will no longer be separately gauge invariant, and only the difference in Eq. (144) remains physically meaningful.

\section{B. Lattice viscosity}

Next, let us turn to the lattice version of the Kubo formula for viscosity in Sec. IV A. For free fermions, our starting point is the lattice stress tensor given in Eq. (57), which we repeat here:

$$
\begin{aligned}
T_{\mathrm{B} 0, \nu}^{\mu}= & \sum_{n m \mathbf{k}} c_{n \mathbf{k}}^{\dagger}\left(\partial_{\mu} f^{n m}(\mathbf{k}) \frac{\sin \mathbf{k} \cdot \mathbf{a}_{\nu}}{\left|\mathbf{a}_{\nu}\right|}\right. \\
& \left.+\frac{i}{2} \tilde{\epsilon}^{\mu}{ }_{\nu} \cos \mathbf{k} \cdot \mathbf{a}_{\mu}\left[f(\mathbf{k}), L_{\mathrm{int}}\right]^{n m}\right) c_{m \mathbf{k}} .
\end{aligned}
$$

The fundamental differences between this and the continuum expression (103) are the replacement of $k_{\mu}$ by $\sin \left(\mathbf{k} \cdot \mathbf{a}_{\mu}\right) /\left|\mathbf{a}_{\mu}\right|$ and the factor of $\cos \mathbf{k} \cdot \mathbf{a}_{\mu}$ in the spin stress. These similarities in structure allow us to repeat much of the same logic as in the continuum case for evaluating expectation values of the stress tensor and commutators. In particular, the single-particle lattice Green's function $G_{n m}(\mathbf{k})$ has a form identical to Eq. (106), with $\epsilon_{\alpha}$ and $U_{\alpha n}$ periodic functions of $\mathbf{k}$. As in Sec. VA, we evaluate the equilibrium stress, Hall elastic modulus, and Hall viscosity for a generic free-fermion lattice system.

\section{Equilibrium stress}

Let us begin by evaluating the ground-state average $\left\langle T_{\mathrm{B} 0, \nu}^{\mu}\right\rangle_{0}$. As in the continuum case, we find, using Eq. (106), that the average of the spin-stress term vanishes, and

$$
\begin{aligned}
\left\langle T_{\mathrm{B} 0, \nu}^{\mu}\right\rangle_{0} & =\sum_{\alpha \mathbf{k}} n_{F}\left[\epsilon_{\alpha}(\mathbf{k})\right] \frac{\sin \mathbf{k} \cdot \mathbf{a}_{\nu}}{\left|\mathbf{a}_{\nu}\right|} \partial^{\mu} \epsilon_{\alpha}(\mathbf{k}) \\
& =\frac{V}{4 \pi^{2}} \sum_{\alpha} \oint d^{2} k n_{F}\left[\epsilon_{\alpha}(\mathbf{k})\right] \frac{\sin \mathbf{k} \cdot \mathbf{a}_{\nu}}{\left|\mathbf{a}_{\nu}\right|} \partial^{\mu} \epsilon_{\alpha}(\mathbf{k}) .
\end{aligned}
$$

Unlike in the continuum, a coordinate transformation such as Eq. (110) does not simplify our calculations. First, in the Brillouin zone, constant energy contours may form noncontractible closed curves, rendering the coordinate transformation ill defined. Second, even for contractible Fermi pockets, we can no longer exploit symmetry to explain the vanishing of the integral due to the sin function. As such, we cannot conclude that the average stress is isotropic in a lattice system, which is reasonable since, in a fundamental sense, a lattice system is generically similar to a solid. Note, however, that in the "lattice gauge theory" continuum limit with $\left|\mathbf{a}_{\mu}\right| \rightarrow 0$, the stress tensor acquires a continuum form, so we recover an isotropic expectation value. This result supports the notion that at wavelengths that are long compared to the lattice spacing (i.e., for metallic systems with small Fermi surfaces), we recover a hydrodynamic picture of electron transport.

However, if we assume that our Hamiltonian has threefold or higher rotational symmetry, we can greatly constrain the average stress. To see this case, we follow the logic of Sec. IV C. Writing, in general,

$$
\left\langle T_{\mathrm{B} 0, \nu}^{\mu}\right\rangle_{0}=T_{\delta} \delta_{\nu}^{\mu}+T_{\epsilon} \tilde{\epsilon}^{\mu}{ }_{\nu}+T_{x} \sigma_{x, \nu}^{\mu}+T_{z} \sigma_{z, \nu}^{\mu},
$$

we see that the coefficients $\left(T_{x}, T_{z}\right)$ must transform in a nontrivial representation of the group of $n$-fold rotations whenever $n \geq 3$. Since these quantities must be scalars by definition, we conclude that $T_{x}=T_{z}=0$ in the presence of more than threefold rotational symmetry. Furthermore, mirror symmetry (along any direction) forces $T_{\epsilon}=0$ by the same logic. 


\section{Stress response}

Let us now consider the Hall response of the lattice Kubo formula Eq. (70). As in the continuum, we separately analyze the contact term and the time-integral contribution to the response function. Starting with the contact term, we will be rather brief. Unlike in the continuum, the lattice strain generators $\mathcal{J}_{\mathrm{L}, \nu}^{\mu}$ do not form a closed algebra. As such, while we can still use the Jacobi identity to write the Hall contribution of the contact term as

$$
\begin{aligned}
C_{\nu}^{\mu \lambda}{ }_{\nu}^{\lambda} & =\frac{1}{2 \omega}\left(\left\langle\left[\left[H_{0}, \mathcal{J}_{\mathrm{L}, \nu}^{\mu}\right], \mathcal{J}_{\mathrm{L}, \rho}^{\lambda}\right]\right\rangle_{0}-\left\langle\left[\left[H_{0}, \mathcal{J}_{\mathrm{L}, \rho}^{\lambda}\right], \mathcal{J}_{\mathrm{L}, \nu}^{\mu}\right]\right\rangle_{0}\right) \\
& =-\frac{1}{2 \omega}\left\langle\left[\left[\mathcal{J}_{\mathrm{L}, \nu}^{\mu}, \mathcal{J}_{\mathrm{L}, \rho}^{\lambda}\right], H_{0}\right]\right\rangle_{0},
\end{aligned}
$$

we cannot reduce this to an expression involving only the ground-state average of the lattice stress tensor. Instead, we have

$$
\begin{aligned}
{\left[\mathcal{J}_{\mathrm{L}, \nu}^{\mu}, \mathcal{J}_{\mathrm{L}, \rho}^{\lambda}\right]=} & -\frac{1}{4} \sum_{\mathbf{k} n} c_{n \mathbf{k}}^{\dagger}\left(\delta_{\rho}^{\mu}\left\{\left\{\partial^{\lambda}, \cos \mathbf{k} \cdot \mathbf{a}_{\mu}\right\}, \sin \mathbf{k} \cdot \mathbf{a}_{\nu}\right\}\right. \\
& \left.-\delta_{\nu}^{\lambda}\left\{\left\{\partial^{\mu}, \cos \mathbf{k} \cdot \mathbf{a}_{\nu}\right\}, \sin \mathbf{k} \cdot \mathbf{a}_{\rho}\right\}\right) c_{n \mathbf{k}} \\
& +\frac{i}{2} \sum_{\mathbf{k} n m} \delta^{\mu \lambda} c_{n \mathbf{k}}^{\dagger} L_{\mathrm{int}}^{n m} c_{m \mathbf{k}} \sin \mathbf{k} \cdot \mathbf{a}_{\mu}\left(\hat{\epsilon}^{\mu}{ }_{\nu} \sin \mathbf{k} \cdot \mathbf{a}_{\rho}\right. \\
& \left.-\hat{\epsilon}^{\mu}{ }_{\rho} \sin \mathbf{k} \cdot \mathbf{a}_{\nu}\right) .
\end{aligned}
$$

We can then insert this expression into Eq. (149). Fortunately, like in the continuum, the internal angular momentum does not contribute to the contact term. We find

$$
\begin{aligned}
C_{\nu \rho}^{\mu \lambda}= & \frac{1}{2 \omega} \sum_{\mathbf{k} n m} G_{n m}(\mathbf{k})\left[\delta_{\rho}^{\mu} \cos \mathbf{k} \cdot \mathbf{a}_{\rho} \sin \mathbf{k} \cdot \mathbf{a}_{\nu} \partial^{\lambda} f_{n m}(\mathbf{k})\right. \\
& \left.-\delta_{\nu}^{\lambda} \cos \mathbf{k} \cdot \mathbf{a}_{\nu} \sin \mathbf{k} \cdot \mathbf{a}_{\rho} \partial^{\mu} f_{n m}(\mathbf{k})\right],
\end{aligned}
$$

which we cannot simplify further, in general.

For the Hall viscosity (121), we are considerably more fortunate. To evaluate the Kubo formula, it is convenient to treat the kinetic and internal angular-momentum contributions to the stress tensor separately. We can write

$$
\left(\eta^{\mathrm{H}}\right)^{\mu}{ }_{\nu \rho}^{\lambda}=\left(\eta_{\mathrm{kin}}^{\mathrm{H}}\right)^{\mu}{ }_{\nu \rho}^{\lambda}+\left(\eta_{\mathrm{int}}^{\mathrm{H}}\right)^{\mu}{ }_{\nu \rho}^{\lambda},
$$

where $\left(\eta_{\text {kin }}^{\mathrm{H}}\right)^{\mu}{ }_{\nu}{ }_{\rho}$ is defined by Eq. (121) with only the kinetic contribution to the stress tensor included. For the kinetic contribution, we can follow the same logic as in Sec. VA. In complete analogy with that section, we find

$\left(\eta_{\text {kin }}^{\mathrm{H}}\right)_{\nu \rho}^{\mu \lambda}=\frac{1}{4 \pi^{2}} \int_{\text {occ }} d^{2} k \sin \mathbf{k} \cdot \mathbf{a}_{\nu} \sin \mathbf{k} \cdot \mathbf{a}_{\rho} \hat{\epsilon}^{\mu \lambda} \operatorname{tr}(\Omega)$.

Thus, like in the continuum, the strain viscosity is related to (periodic) moments of the trace of the Berry curvature. Next, we can compute the internal angular-momentum contributions to the viscosity. Unlike in the continuum, these contribute to both the barred and unbarred viscosity coefficients (since, as noted in Sec. III, the internal angularmomentum contribution to the stress tensor is not purely antisymmetric on the lattice). Following the computation in Sec. VA, we find that at zero temperature,

$$
\begin{aligned}
\left(\eta_{\text {int }}^{\mathrm{H}}\right)^{\mu}{ }_{\nu \rho}^{\lambda}= & \sum_{\alpha \beta} \frac{1}{4 \pi^{2}} \int d^{2} k\left[n\left(\epsilon_{\alpha}\right)-n\left(\epsilon_{\beta}\right)\right] \\
& \times L_{\text {int }}^{\alpha \beta}\left(\hat{\epsilon}_{\rho}^{\lambda} \cos \mathbf{k} \cdot \mathbf{a}_{\lambda} \sin \mathbf{k} \cdot \mathbf{a}_{\nu} A_{\alpha \beta}^{\mu}\right. \\
& \left.-\hat{\epsilon}^{\mu}{ }_{\nu} \cos \mathbf{k} \cdot \mathbf{a}_{\mu} \sin \mathbf{k} \cdot \mathbf{a}_{\rho} A_{\alpha \beta}^{\lambda}\right) .
\end{aligned}
$$

As in the continuum, we have that the kinetic viscosity (153) does not contribute to the Hall tensor (83) and hence does not contribute to viscous forces; the internal angular momentum is responsible for all nondissipative viscous forces that enter the equations of motion. One important difference on the lattice, however, is that the internal viscosity $\left(\eta_{\mathrm{int}}^{\mathrm{H}}\right)^{\mu}{ }_{\nu}{ }_{\rho}$ on the lattice contributes to both the barred and unbarred viscosity coefficients, due to the extra cosine factors in Eq. (154).

\section{EXAMPLE SYSTEMS}

\section{A. Continuum massive Dirac fermion}

As a first example, let us consider a massive Dirac fermion in two dimensions, with the Hamiltonian

$$
H=\sum_{k \sigma \sigma^{\prime}} c_{\sigma \mathbf{k}}^{\dagger}\left(\vec{d}(\mathbf{k}) \cdot \vec{\sigma}^{\sigma \sigma^{\prime}}\right) c_{\sigma^{\prime} \mathbf{k}},
$$

where $\vec{d}(\mathbf{k})$ is a three-vector of functions $\vec{d}=\left(d_{x}, d_{y}, d_{z}\right) \equiv$ $\left(\mathbf{d}, d_{z}\right), \vec{\sigma}=\left(\sigma_{x}, \sigma_{y}, \sigma_{z}\right) \equiv\left(\boldsymbol{\sigma}, \sigma_{z}\right)$, and we view this internal degree of freedom as a real spin. Note that we use $\mathbf{v}$ for twodimensional vectors, and $\vec{v}$ for three-dimensional vectors. The single-particle eigenvalues and eigenfunctions of this Hamiltonian have the well-known form

$$
\begin{aligned}
& | \pm \mathbf{k}\rangle=\sum_{\sigma} u_{\sigma \mathbf{k}} c_{\sigma \mathbf{k}}^{\dagger}|0\rangle, \\
& H| \pm \mathbf{k}\rangle= \pm E_{\mathbf{k}}| \pm \mathbf{k}\rangle,
\end{aligned}
$$

with

$$
\begin{gathered}
u_{\uparrow \mathbf{k}}=\left(\cos \frac{\theta}{2}, e^{i \phi} \sin \frac{\theta}{2}\right), \\
u_{\downarrow \mathbf{k}}=\left(\sin \frac{\theta}{2},-e^{i \phi} \cos \frac{\theta}{2}\right), \\
E_{k}=|\vec{d}(\mathbf{k})|, \\
\tan \theta=\frac{|\mathbf{d}|}{m},
\end{gathered}
$$




$$
\tan \phi=\frac{d_{y}}{d_{x}} .
$$

Since we view $\sigma, \sigma^{\prime}$ as real spin, rotations in the plane are generated by both the kinetic angular-momentum and the internal angular-momentum operator given by

$$
L_{\text {int }}=\frac{1}{2} \sigma^{z}
$$

Using Eq. (103), we can compute the Belinfante stress tensor for this model. We find

$$
\begin{aligned}
T_{\mathrm{B}, \nu}^{\mu} & =\sum_{\mathbf{k} \sigma \sigma^{\prime}} c_{\sigma \mathbf{k}}^{\dagger}\left(k_{\nu} \partial^{\mu} \vec{d} \cdot \vec{\sigma}^{\sigma \sigma^{\prime}}+\frac{1}{2} \epsilon^{\mu}{ }_{\nu}\left(\mathbf{d} \times \boldsymbol{\sigma}^{\sigma \sigma^{\prime}}\right)\right) c_{\sigma^{\prime} \mathbf{k}} \\
& \equiv \sum_{\mathbf{k} \sigma \sigma^{\prime}} c_{\sigma \mathbf{k}}^{\dagger}\left[T^{\sigma \sigma^{\prime}}\right]_{\mathrm{B}, \nu}^{\mu}(\mathbf{k}) c_{\sigma^{\prime} \mathbf{k}} .
\end{aligned}
$$

We use this result to evaluate the viscosity for both isotropic and anisotropic systems.

\section{Isotropic Dirac point}

Let us first focus on the isotropic case

$$
\vec{d}=\left(v_{\mathrm{F}} \mathbf{k}, m\right)
$$

with $m$ a constant mass. This choice of $\vec{d}$ corresponds to an isotropic massive Dirac fermion in two dimensions. The stress tensor in our formalism for this case coincides with the usual Belinfante tensor for a Dirac theory [39]

$$
T_{\mathrm{B}, \nu}^{\mu}(\mathbf{k})=\frac{v_{\mathrm{F}}}{2}\left(k_{\nu} \sigma^{\mu}+k_{\mu} \sigma^{\nu}\right)
$$

where, since this is a flat-space expression, the distinction between upper and lower indices is immaterial. We have also suppressed the $\sigma, \sigma^{\prime}$ indices on the Pauli matrices for notational convenience. Since this system is invariant under continuous rotational symmetries, and the stress tensor is symmetric, we can make use of Eqs. (126)-(128) and (133) - (135) to evaluate the viscosity. In fact, the symmetry of the problem immediately implies that only $\eta^{\mathrm{H}}$ can be nonzero. Let us consider the Fermi level $E_{\mathrm{F}}$ in the lower band of the model. We can write the Berry curvature for this band as

$$
\begin{aligned}
\Omega_{-} & =\frac{1}{2} \sum_{\mu \nu} \epsilon_{\mu \nu} \sin \theta \partial^{\mu} \theta \partial^{\nu} \phi \\
& =\frac{m v_{\mathrm{F}}^{2}}{2 E_{\mathbf{k}}^{3}} .
\end{aligned}
$$

Substituting this result into Eq. (126), we find

$$
\begin{aligned}
\eta^{\mathrm{H}} & =\frac{1}{16 \pi^{2}} \int_{\mathrm{occ}} d^{2} k|\mathbf{k}|^{2} \Omega_{-} \\
& =\frac{1}{4 \pi^{2}} \int_{\mathrm{occ}} d^{2} k \frac{m v_{\mathrm{F}}^{2}|\mathbf{k}|^{2}}{8 E_{\mathbf{k}}^{3}},
\end{aligned}
$$

consistent with the known result [30,59]. Because the energy spectrum of a Dirac fermion is unbounded from below, we need to introduce a momentum cutoff to evaluate this expression. It is perhaps more useful, however, to examine the differential viscosity

$$
\frac{d \eta^{\mathrm{H}}}{d E_{\mathrm{F}}}=\frac{m}{16 \pi E_{\mathrm{F}}}\left(m^{2}-E_{\mathrm{F}}^{2}\right),
$$

the derivative of the Hall viscosity as a function of chemical potential. Since we assume that only the lower band is occupied, this expression is valid only when $E_{\mathrm{F}} \leq-|m|$. For $-|m| \leq E_{\mathrm{F}} \leq|m|$, we have that

$$
\frac{d \eta^{\mathrm{H}}}{d E_{\mathrm{F}}}=0 .
$$

Finally, when the Fermi surface is in the upper band $\left(E_{\mathrm{F}} \geq|m|\right)$,

$$
\frac{d \eta^{\mathrm{H}}}{d E_{\mathrm{F}}}=\frac{m}{16 \pi E_{\mathrm{F}}}\left(E_{\mathrm{F}}^{2}-m^{2}\right)
$$

where we have used the fact that $\Omega_{+}=-\Omega_{-}$.

\section{2. $C_{4}$ warping}

Next, let us consider an anisotropic Dirac Hamiltonian with only fourfold rotational symmetry. The simplest such model is given by

$$
\begin{gathered}
d_{x}=v_{\mathrm{F}} k_{x}+u k_{x}^{3}, \\
d_{y}=v_{\mathrm{F}} k_{y}+u k_{y}^{3}, \\
d_{z}=m .
\end{gathered}
$$

The stress tensor determined from Eq. (165) is

$$
\begin{aligned}
T_{\mathrm{B}, \nu}^{\mu}(\mathbf{k})= & \frac{v_{\mathrm{F}}}{2}\left(k_{\mu} \sigma^{\nu}+k_{\nu} \sigma^{\mu}\right)+\frac{3 u}{2}\left(k_{\nu} k_{\mu}^{2} \sigma^{\mu}+k_{\mu} k_{\nu}^{2} \sigma^{\nu}\right) \\
& +\frac{u}{2} \epsilon^{\mu}{ }_{\nu}\left[k_{y} \sigma^{x}\left(3 k_{x}^{2}-k_{y}^{2}\right)-k_{x} \sigma^{y}\left(3 k_{y}^{2}-k_{x}^{2}\right)\right] .
\end{aligned}
$$

Since this model does not have continuous rotational symmetry, even the Belinfante stress tensor has an explicit antisymmetric contribution. Since we still have fourfold rotational symmetry, however, we expect the only nonzero viscosities to be $\eta^{\mathrm{H}}$ and $\bar{\eta}^{\mathrm{H}}$. To compute these viscosities, we note that, in addition to Eqs. (156)-(162), 
the expression (168) also holds true even for this anisotropic model. Using this result, along with the expressions (126) and (133) or directly from the Kubo formula (70), we find that when the chemical potential is in the valence band,

$$
\begin{aligned}
\eta^{\mathrm{H}}= & \frac{1}{4 \pi^{2}} \int_{\mathrm{occ}} d^{2} k \frac{m|\mathbf{k}|^{2}\left(3 u k_{x}^{2}+v_{\mathrm{F}}\right)\left(3 u k_{y}^{2}+v_{\mathrm{F}}\right)}{8 E_{\mathbf{k}}^{3}}, \\
\bar{\eta}^{\mathrm{H}}= & \frac{1}{4 \pi^{2}} \int_{\mathrm{occ}} d^{2} k \frac{m u}{8 E_{\mathbf{k}}^{3}}\left\{3|\mathbf{k}|^{2}\left[\left(k_{x}^{2}-k_{y}^{2}\right)^{2}-2 k_{x}^{2} k_{y}^{2}\right]\right. \\
& \left.+v_{F}\left[\left(k_{x}^{2}-k_{y}^{2}\right)^{2}-4 k_{x}^{2} k_{y}^{2}\right]\right\} .
\end{aligned}
$$

As in the isotropic case, these integrals require a momentum cutoff to be evaluated, although in the interest of brevity, we will not pursue this further in this section. The physically relevant combination $\eta_{\text {total }}^{\mathrm{H}}=\eta^{\mathrm{H}}+\bar{\eta}^{\mathrm{H}}$ that determines the Hall tensor (83) and viscous forces (78) is then

$\eta_{\text {total }}^{\mathrm{H}}=\frac{m}{4 \pi^{2}} \int_{\mathrm{occ}} d^{2} k \frac{3 u^{2}\left(k_{x}^{6}+k_{y}^{6}\right)+4 u v_{\mathrm{F}}\left(k_{x}^{4}+k_{y}^{4}\right)+v_{\mathrm{F}}^{2}|\mathbf{k}|^{2}}{8 E_{\mathbf{k}}^{3}}$.

We see that in addition to the isotropic contribution proportional to $|\mathbf{k}|^{2}$, the integrand also contains the rotational-symmetry breaking functions $k_{x}^{4}+k_{y}^{4}$ and $k_{x}^{6}+k_{y}^{6}$, which make the fourfold rotational symmetry manifest.

\section{3. $C_{2}$ symmetry}

Finally, we consider a simple model with only twofold rotational symmetry, by taking

$$
\begin{gathered}
d_{x}=v_{\mathrm{F}}^{x} k_{x}, \\
d_{y}=v_{\mathrm{F}}^{y} k_{y}, \\
d_{z}=m .
\end{gathered}
$$

Because this system differs by only a momentum rescaling from an isotropic Dirac point, the calculation of the viscosity coefficients proceeds in much the same way as in Sec. VI A 1, with the exception being that now the $\bar{\eta}^{H}$ viscosity is nonzero. Although the stress tensor is now

$$
T_{\mathbf{B}, \nu}^{\mu}(\mathbf{k})=k_{\nu} \sigma^{\mu}\left(v_{\mathrm{F}}^{\mu}-\frac{1}{2} v_{\mathrm{F}}^{\nu}\right)+\frac{1}{2} v_{\mathrm{F}}^{\nu} k^{\mu} \sigma^{\nu},
$$

which contains an explicit antisymmetric contribution, we find for the total Hall viscosity an analogous expression to the unbarred Hall viscosity for the isotropic case in Sec. VI A 1. It is given by

$$
\begin{aligned}
\eta_{\text {total }}^{\mathrm{H}} & \equiv \eta^{\mathrm{H}}+\bar{\eta}^{\mathrm{H}}=\frac{1}{16 \pi^{2}} \int_{\mathrm{occ}} d^{2} k|\mathbf{k}|^{2} \Omega_{-} \\
& =\frac{1}{4\left\langle v_{\mathrm{F}}\right\rangle_{\mathrm{g}}^{2} \pi^{2}} \int_{\mathrm{occ}} d^{2} u \frac{m|\mathbf{u}|^{2}}{8 E_{\mathbf{u}}^{3}} .
\end{aligned}
$$

We have introduced transformed coordinates $u_{x}=v_{F}^{x} k_{x}$ and $u_{y}=v_{F}^{y} k_{y}$, and we have also introduced the geometric mean

$$
\left\langle v_{\mathrm{F}}\right\rangle_{g} \equiv \sqrt{v_{\mathrm{F}}^{x} v_{\mathrm{F}}^{y}}
$$

Thus, we see that for this simple $C_{2}$ symmetric model, the total Hall viscosity matches the isotropic result upon replacing the isotropic Fermi velocity with the geometric mean of the two anisotropic Fermi velocities.

In the next section, we examine a lattice regularization for the Dirac Hamiltonian (155), which is of considerable interest in its own right as a model for a Chern insulator. We highlight what differs between the lattice and continuum models and show how to take the continuum limit of the lattice viscosities in the limit of large bandwidth.

\section{B. Lattice models for a Chern insulator}

We now move to our first example without full translational invariance - a square lattice Chern insulator. For our Bravais lattice vectors, we take

$$
\mathbf{a}_{1}=a \hat{\mathbf{x}}, \quad \mathbf{a}_{2}=a \hat{\mathbf{y}},
$$

and thus we can avoid the complications associated with non-Cartesian bases. This model represents the lattice regularization of the massive Dirac fermion in the previous section-heuristically, we map the momenta into crystal momenta $k_{\mu} \rightarrow \sin \left(\mathbf{k} \cdot \mathbf{a}_{\mu}\right)=\sin \left(k_{\mu} a\right)$ and add a momentum-dependent mass to the model. We continue to use the tight-binding Hamiltonian form of Eq. (155),

$$
\begin{aligned}
& d_{x}=t \sin \left(k_{x} a\right), \\
& d_{y}=t^{\prime} \sin \left(k_{y} a\right), \\
& d_{z}=m-r\left\{\cos \left(k_{x} a\right)+\cos \left(k_{y} a\right)\right\} .
\end{aligned}
$$

This model still has internal degrees of freedom that need to be taken into account when considering rotations of the system, meaning that the generator of rotations is the full angular-momentum operator (17), and the proper rotation operator is thus

$$
\hat{R}(\phi)=R_{\mathrm{orb}}(\phi) \otimes e^{i \phi \sigma^{2} / 2} .
$$

The orbital rotation matrix is of the usual form $R_{\text {orb }}(\phi) \in$ $S O(2)$ as in Eq. (15). The square lattice Chern insulator model we have described is always symmetric under twofold rotations. The point group that leaves the Hamiltonian invariant is therefore $C_{2}$. When $t=t^{\prime}$, the model acquires a fourfold rotational symmetry as well, expanding the point group to $C_{4}$. The model also always has $P T$ symmetry, which can be represented as 


$$
\Delta(P T)=\sigma_{z} \mathcal{K},
$$

where $K$ is complex conjugation, and $P T$ takes $k_{x} \rightarrow-k_{x}$ and leaves $k_{y}$ invariant. For any $m \neq 0, \pm 2 r$, this model is fully gapped. When $|m| \geq 2 r$, the valence and conduction bands have Chern number zero, while when $|m| \leq 2 r$, the valence and conduction bands each have Chern number of magnitude 1 .

The stress tensor, computed with Eq. (145), is then given in components by

$$
T_{\mathrm{B}, \rho}^{\lambda}=\sum_{\mathbf{k} \sigma \sigma^{\prime}} c_{\sigma \mathbf{k}}^{\dagger}\left[T^{\sigma \sigma^{\prime}}\right]_{\mathrm{B}, \nu}^{\mu}(\mathbf{k}) c_{\sigma^{\prime} \mathbf{k}}
$$

with components (repeated indices are not summed over)

$$
\begin{aligned}
\hat{T}_{\mathrm{B}, \rho}^{\lambda}(\mathbf{k})= & t^{\lambda} \cos \left(k_{\lambda} a\right) \sin \left(k_{\rho} a\right) \sigma^{\lambda}+r \sin \left(k_{\lambda} a\right) \sin \left(k_{\rho} a\right) \sigma^{z} \\
& +\frac{1}{2} \cos \left(k_{\lambda} a\right)\left[t^{\lambda} \sin \left(k_{\lambda} a\right) \sigma^{\rho}-t^{\rho} \sin \left(k_{\rho} a\right) \sigma^{\lambda}\right] \\
\equiv & {\left[\hat{T}^{\mathrm{kin}}(\mathbf{k})\right]_{\rho}^{\lambda}+\left[\hat{T}^{\mathrm{spin}}(\mathbf{k})\right]_{\rho}^{\lambda} . }
\end{aligned}
$$

The first two terms above are the stress tensor calculated from the purely kinetic part of the strain generator; they are therefore labeled $\left(\hat{T}^{\mathrm{kin}}\right)_{\rho}^{\lambda}$, whereas the spin contribution is given by $\left(\hat{T}^{\text {spin }}\right)_{\rho}^{\lambda}$. We make this distinction to stress the importance of including the spin contribution for viscosity computations-showing the difference between what we call the kinetic viscosity ( $\eta$ calculated from solely the strain part of the stress tensor) and the overall viscosity. We can tailor the Kubo formula (70) to our system by writing

$$
\begin{gathered}
{\left[\eta^{\mathrm{H}}(\mathbf{k})\right]_{\rho^{\mu}{ }_{\nu}}^{\lambda}=-\frac{\Im\left\{\left[T^{-+}(\mathbf{k})\right]_{\mathrm{B}, \rho}^{\lambda}\left[T^{+-}(\mathbf{k})\right]_{\mathrm{B}, \nu}^{\mu}\right\}}{2 \epsilon_{\mathbf{k}}^{2}},} \\
\left(\eta^{\mathrm{H}}\right)^{\lambda} \rho_{\rho}^{\mu}{ }_{\nu}=\frac{1}{4 \pi^{2}} \int_{\mathrm{occ}} d^{2} k\left[\eta^{\mathrm{H}}(\mathbf{k})\right]_{\rho^{\mu}{ }_{\nu}}^{\lambda} .
\end{gathered}
$$

Above, and henceforth, we use the notation

$$
\left[T^{-+}(\mathbf{k})\right]_{\mathrm{B}, \rho}^{\lambda}=\left\langle-, \mathbf{k}\left|\left[T_{\mathrm{B}, \rho}^{\lambda}(\mathbf{k})\right]\right|+, \mathbf{k}\right\rangle .
$$

We focus on the linear combinations of the viscosity tensor that enter into the Hall tensor (83). For this gapped, timereversal odd model, the isotropic contribution to the Hall tensor is given by the sum of the two isotropic Hall viscosities:

$$
\eta_{\text {total }}^{H}=\eta^{\mathrm{H}}+\bar{\eta}^{H}=\frac{1}{2}\left(\eta_{1}^{1}{ }_{1}{ }_{2}-\eta_{2}^{2}{ }_{2}{ }_{1}\right) .
$$

In general, the anisotropic components $\gamma$ and $\bar{\gamma}$ may also be nonzero, and they are given by the opposite combination of components,

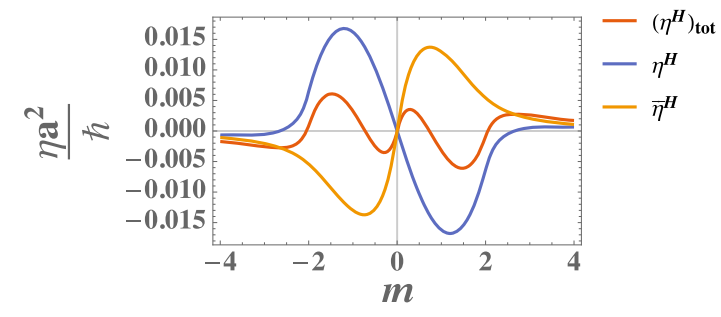

FIG. 1. A plot of the total Hall viscosity $\eta_{\text {total }}^{\mathrm{H}}, \eta^{\mathrm{H}}$, and $\bar{\eta}^{H}$ as a function of mass $m \in[-4,4]$ for the $C_{4}$ symmetric Chern insulator. We fix the hopping parameters $t=r=1$ and set the lattice constant $a=1$. The viscosity is measured in units of $\hbar / a^{2}$. We see that each viscosity is odd in the mass parameter $m$, and all go to zero as $|m| \rightarrow \infty$.

$$
\gamma_{\text {total }}=\gamma+\bar{\gamma}=\frac{1}{2}\left(\eta_{1}^{1}{ }_{1}{ }_{2}+\eta^{2}{ }_{2}{ }_{1}\right)
$$

We can view the above as a measure for the anisotropy of a system-in particular, it represents how far away a system is from having fourfold rotation symmetry.

\section{1. $C_{4}\left(t=t^{\prime}\right)$ system}

We start by considering the $C_{4}$ symmetric system with hoppings $t=t^{\prime}$. First, we note that the Hall modulus (151) vanishes since the integrand is odd in momentum. The total lattice Hall viscosity computed from Eqs. (196) and (198) is a nontrivial expression, which we plot as a function of $m$ in Fig. 1. As in Ref. [30], we see that $\eta^{\mathrm{H}}, \bar{\eta}^{\mathrm{H}}$, and $\eta_{\text {total }}^{\mathrm{H}}$ are all smooth functions of $m$ across all phase boundaries $m=0, \pm 2 r$.

As we saw in Eq. (153), if instead we focus on the contribution to the viscosity from the kinetic stress, we find that the contribution $\eta_{\text {kin }}^{\mathrm{H}}+\bar{\eta}_{\text {kin }}^{\mathrm{H}} \equiv\left(\eta_{\text {total }}^{\mathrm{H}}\right)_{\text {kin }}$ to the Hall tensor has the property that

$$
\left(\eta_{\text {total }}^{\mathrm{H}}\right)_{\text {kin }}=0 \leftrightarrow \eta_{\text {kin }}^{H}=-\bar{\eta}_{\text {kin }}^{H} .
$$

This property was computed in Ref. [30].

Continuum limit.-To extract some of the underlying physics of the problem, we consider the continuum limit of this model by approximating the lattice fermion operators by slowly varying field operators $\psi_{n i}$ defined around the Dirac points in momentum space $\mathbf{K}_{i}=\left(k_{x}, k_{y}\right)=$ $\{(0,0),(\pi, 0),(0, \pi),(\pi, \pi)\}$ :

$$
c_{n \mathbf{R}} \approx \sum_{i} e^{i \mathbf{K}_{i} \cdot \mathbf{R}} \psi_{n i}(\mathbf{R}) .
$$

We can proceed to compute the viscosity in this language but must be careful how we do so since the lattice strain generator (51) has momentum dependence that much be considered to find the proper stress tensor (194). To proceed, we independently expand the lattice Hamiltonian and stress tensor, compute the viscosity around each Dirac point, and 
then finally sum up the resulting coefficients to find the continuum Hall viscosity. To first order, the Hamiltonian for each Dirac point is given by

$$
H^{(i)}=v_{F}\left(\alpha_{x}^{i} k_{x} \sigma^{x}+\alpha_{y}^{i} k_{y} \sigma^{y}\right)+M^{i} \sigma^{z},
$$

where $v_{F} \equiv t a$ and the sign coefficients about each Dirac point are given by $\alpha_{x}^{i}=\{1,-1,1,-1\}$ and $\alpha_{y}^{i}=\{1,1,-1,-1\}$, and the Dirac mass changes across the points as $M^{i}=\{m-2 r, m, m, m+2 r\}$. The dispersion for each of these points is of the same form,

$$
\epsilon_{\mathbf{k}}^{(i)}=\sqrt{v_{F}^{2}|\mathbf{k}|^{2}+\left(M^{i}\right)^{2}} .
$$

The expansion of this tensor around the Dirac points can be written as

$$
\begin{aligned}
\left(T^{(i)}\right)_{\mathrm{B}, \rho}^{\lambda}(\mathbf{k}) & =v_{F}\left[\alpha_{\lambda}^{i} \alpha_{\rho}^{i} \sigma^{\lambda} k_{\rho}+\frac{1}{2} \alpha_{\lambda}^{i}\left(\alpha_{\lambda}^{i} k_{\lambda} \sigma^{\rho}-\alpha_{\rho}^{i} k_{\rho} \sigma^{\lambda}\right)\right] \\
& =\frac{v_{F}}{2} \alpha_{\lambda}^{i}\left[\alpha_{\rho}^{i} \sigma^{\alpha} k_{\rho}+\alpha_{\lambda}^{i} k_{\lambda} \sigma^{\rho}\right] .
\end{aligned}
$$

The resulting viscosity can be easily computed, noting that the Pauli matrices satisfy $\operatorname{Im}\left[\left(\sigma^{a}\right)^{-+}\left(\sigma^{b}\right)^{+-}\right]=$ $M \epsilon_{b a} / \epsilon_{\mathbf{k}}$,

$$
\begin{aligned}
\left(\eta^{(i)}\right)^{\lambda}{ }_{\rho}^{\mu}{ }_{\nu}= & \frac{v_{F}^{2} M^{i}}{4 \epsilon_{\mathbf{k}}^{3}}\left[\alpha_{\lambda}^{i} \alpha_{\rho}^{i} \alpha_{\mu}^{i} \alpha_{\nu}^{i} k_{\rho} k_{\nu} \epsilon_{\nu}^{\lambda}+\alpha_{\lambda}^{i} \alpha_{\rho}^{i} k_{\rho} k_{\mu} \epsilon_{\nu}^{\lambda}\right. \\
& \left.+\alpha_{\mu}^{i} \alpha_{\nu}^{i} k_{\lambda} k_{\nu} \epsilon_{\rho}{ }^{\mu}+k_{\lambda} k_{\mu} \epsilon_{\rho}{ }^{\mu}\right] .
\end{aligned}
$$

Still, the only possible nonzero viscosities are $\eta^{\mathrm{H}}$ and $\bar{\eta}^{H}$, and we need to consider their sum (198). For the expansion, we need to sum over all the Dirac points. The general viscosity integrand across the Dirac points is then given by

$$
\left[\eta_{\text {total }}^{H}(\mathbf{k})\right]^{(i)}=\left[\eta^{\mathrm{H}}(\mathbf{k})+\bar{\eta}^{H}(\mathbf{k})\right]^{(i)}=\frac{v_{F}^{2} M^{i}}{8 \epsilon_{\mathbf{k}}^{3}}|\mathbf{k}|^{2} .
$$

Equation (206) shows that each Dirac point contributes to $\eta_{\text {total }}^{\mathrm{H}}$, identically to Eq. (171), for an isolated isotropic Dirac point in the continuum. This result is distinct from that in Ref. [30] for the Hall viscosity obtained using only the kinetic stress tensor and focusing only on the isotropic viscosity. The distinction arises due to our careful treatment of the spin stress. We note that, as shown in the Appendix, there exists an alternative definition for the spin stress that reproduces the results of Ref. [30] for the viscosity of the Chern insulator model when both $\eta^{\mathrm{H}}$ and $\bar{\eta}^{\mathrm{H}}$ are computed. However, this case comes at the expense of the simplicity of Eq. (41) in real space.

We expect this continuum approximation to be accurate when the mass gap at each Dirac point is small compared to the bandwidth, meaning $t \gg m, r$. In this limit, the lowenergy behavior of the model is approximately that of four independent massive Dirac points. To verify this behavior,

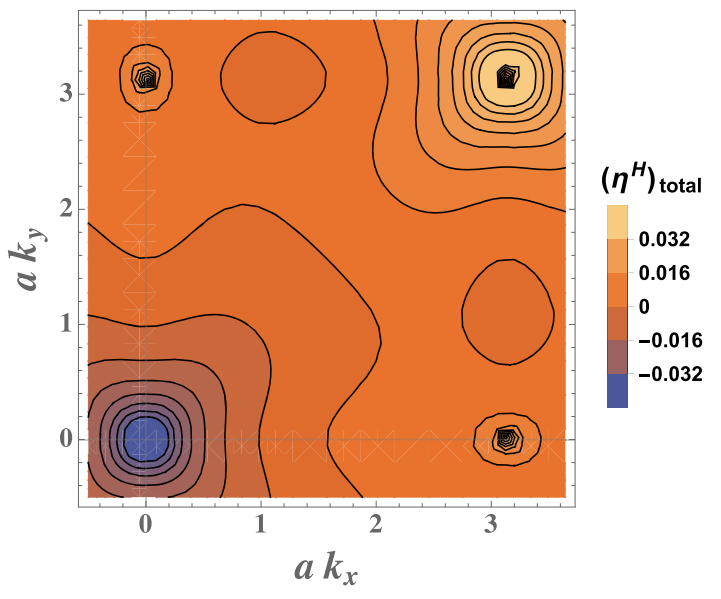

FIG. 2. Contour plot of the density $\eta_{\text {total }}^{\mathrm{H}}(\mathbf{k})$ for the $C_{4}$ symmetric Chern insulator. The plot is shown with the hopping parameters $t=t^{\prime}=20, m=0.25$, and $r=1$.

we show in Fig. 2 a plot of the density $\eta_{\text {total }}^{\mathrm{H}}(\mathbf{k})$ for $t=$ $t^{\prime}=20, m=r=1$. We see that near the Dirac points, the viscosity is isotropic and matches the form of the integrand in Eq. (171).

Returning to Eq. (206), we can introduce a momentum cutoff $\Lambda$, integrate over momentum, and sum over the Dirac points to obtain

$$
\eta_{\mathrm{total}}^{H}=\frac{1}{2} \sum_{i}\left[\frac{M^{i} \Lambda}{8 \pi v_{F}}-\frac{M^{i}\left|M^{i}\right|}{4 \pi v_{F}^{2}}\right] .
$$

The momentum cutoff $\Lambda$ is introduced to perform the integrals about each Dirac point, and the overall viscosity is given by a cutof-dependent piece and a universal part,

$$
\eta_{\mathrm{total}}^{H}=\left[\frac{m \Lambda}{4 \pi v_{F}}-\sum_{i} \frac{M^{i}\left|M^{i}\right|}{8 \pi v_{F}^{2}}\right]
$$

Unlike in Ref. [30], however, we see that the contributions to the viscosity above the momentum cutoff cannot be neglected. If we focus on the barred and unbarred Hall viscosities separately, we see that the end points $(0,0)$ and $(\pi, \pi)$ are described by fully isotropic theories, so the unbarred Hall viscosity is the only nonzero contribution $\left(\eta_{\text {total }}^{\mathrm{H}}\right)^{(0,3)}=\eta^{\mathrm{H}}$. At the corner points, however, $(\pi, 0)$ and $(0, \pi),\left(\eta_{\text {total }}^{\mathrm{H}}\right)^{(1,2)}=\bar{\eta}^{H}$. Thus, the total viscosity from each Dirac point is the same, but the isotropic points express their viscosity through $\eta^{\mathrm{H}}$, and the anisotropic ones express their viscosity through the rotational-symmetry-breaking coefficient $\bar{\eta}^{H}$. Examining the contour plot in Fig. 2, we can interpret the roll of the momentum cutoff $\Lambda$ as limiting the domain of integration to the regions of the Brillouin zone, where the viscous density $\eta_{\text {total }}^{\mathrm{H}}(\mathbf{k})$ is concentrated near the (massive) Dirac points.

Therefore, we see that the barred viscosity is nonzero at some of the Dirac points, and considering it is crucial for achieving the result Eq. (208). If we were to consider only 
the unbarred viscosity $\eta^{\mathrm{H}}$, we would be neglecting half of the physical effect of the viscosity. Our results here should be contrasted with those of Ref. [30], which computed the kinetic viscosity $\eta_{\text {kin }}^{\mathrm{H}}$ only. This viscosity does not contribute to viscous forces since, as mentioned previously, $\bar{\eta}_{\text {kin }}^{\mathrm{H}}=-\eta_{\text {kin }}^{\mathrm{H}}$. Nevertheless, those authors found that the continuum limit expression for $\eta_{\text {kin }}^{\mathrm{H}}$ is cutoff independent for the Chern insulator. Here, on the other hand, we find the equally appealing result that $\eta_{\mathrm{total}}^{\mathrm{H}}$ for the Chern insulator reduces, in the continuum limit, to the total viscosity of four uncoupled isotropic massive Dirac points. This result is consistent with the expansion (201) about the four massive Dirac points in the model.

\section{2. $C_{2}$ anisotropy and comments on physical responses}

With the intuition from the fourfold rotation-invariant case, we now consider the general model in Eq. (190). We first mention that this model realizes the anisotropic viscosity components $\gamma$ and $\bar{\gamma}$ in addition to $\eta_{\text {total }}^{\mathrm{H}}$ (all others are forbidden by $P T$ symmetry). We plot the total anisotropic viscosity $\gamma_{\text {total }}$ as a function of $m$ in Fig. 3.

Continuum limit.-Again, we consider the Dirac point expansion of the model, now noting that we can absorb the anisotropy into a nonuniform Fermi velocity $\mathbf{v}_{\mathbf{F}}=\left(t a, t^{\prime} a\right)$. Here, we find an analogous result for the physical viscosity:

$$
\left[\eta_{\text {total }}(\mathbf{k})\right]^{(i)}=\frac{M^{i}}{8 \epsilon_{\mathbf{k}}^{3}}\left[\left(v_{F}^{x} k_{x}\right)^{2}+\left(v_{F}^{y} k_{y}\right)^{2}\right] .
$$

If we make the transformations $u_{x}=v_{F}^{x} k_{x}$ and $u_{y}=v_{F}^{y} k_{y}$, then the viscosity takes the same form as the isotropic case,

$$
\begin{aligned}
\left(\eta_{\text {total }}(\mathbf{k})\right]^{(i)} & =\frac{M^{i}}{8} \frac{\left(u_{x}^{2}+u_{y}^{2}\right)}{\left[u_{x}^{2}+u_{y}^{2}+\left(M^{i}\right)^{2}\right]^{3 / 2}} \\
& =\frac{M^{i}}{8} \frac{|\mathbf{u}|^{2}}{\left[|\mathbf{u}|^{2}+\left(M^{i}\right)^{2}\right]^{3 / 2}} .
\end{aligned}
$$

In the last line, we have moved to polar coordinates in $u_{x}, u_{y}$. Performing the integration yields a result analogous to Eq. (207),

$$
\left(\eta_{\text {total }}^{\mathrm{H}}\right)^{(i)}=\frac{1}{2} \sum_{i}\left[\frac{m \tilde{\Lambda}}{8 \pi\left\langle v_{F}\right\rangle_{g}}-\sum_{i} \frac{M^{i}\left|M^{i}\right|}{4 \pi\left\langle v_{F}\right\rangle_{g}^{2}}\right] .
$$

As in the case of the isolated $C_{2}$ Dirac fermion, the viscosity in this case is proportional to the inverse geometric mean of Fermi velocities (188) when the Fermi velocity is nonuniform. The cutoff in this case is of the form

$$
\tilde{\Lambda}=a \sqrt{t^{2} \Lambda_{x}+t^{\prime 2} \Lambda_{y}^{2}}
$$

where $\Lambda_{x, y}$ are momentum cutoffs. We are left with an expression analogous to the $C_{4}$ result, simply replacing the cutoff and Fermi velocity with their anisotropic counterparts in $C_{2}$.

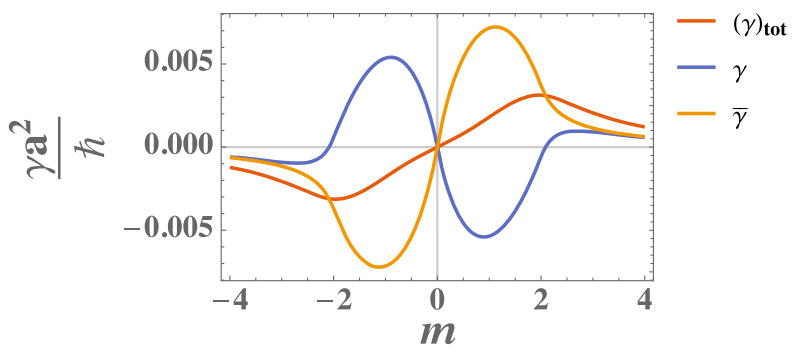

FIG. 3. The anisotropic viscosities $\gamma_{\text {total }}, \gamma$, and $\bar{\gamma}$ as a function of the mass $m \in[-4,4]$ for the $C_{2}$ symmetric Chern insulator. The hoppings are fixed to be $t=1.5$ and $t=r=1$, while the lattice constant is again $a=1$. We see that all viscosities are odd in $m$.

In Fig. 3, we see that the lattice version of this model gives rise to anomalous viscosities $\gamma$ and $\bar{\gamma}$, which changes when moving to the long-wavelength continuum expansion. When summed over the Dirac points and transformed to variables $u_{x}$ and $u_{y}$, we find for both coefficients that, strikingly,

$$
\begin{aligned}
\gamma(\mathbf{k})=-\bar{\gamma}(\mathbf{k}) & =\sum_{i} \gamma^{(i)}(\mathbf{k})=\frac{m}{16 \epsilon_{\mathbf{u}}^{3}}\left(u_{x}^{2}-u_{y}^{2}\right) \\
& =\frac{m|\mathbf{u}|^{2}}{16 \epsilon_{\mathbf{u}}^{3}} \cos \left(2 \theta_{\mathbf{u}}\right) .
\end{aligned}
$$

As these expressions transform nontrivially under rotations of the new coordinates, they vanish upon integration over any isotropic surface. We thus have that $\bar{\gamma}=\gamma=0$ in the continuum limit.

As in the case of the isotropic viscosity, we expect the continuum expansion to capture the behavior of the viscosity when the bandwidth is large compared to the gap. We plot the density $\gamma_{\text {total }}$ for hopping parameters $t=t^{\prime}+1=20, r=1, m=1$ in Fig. 4. We see that the

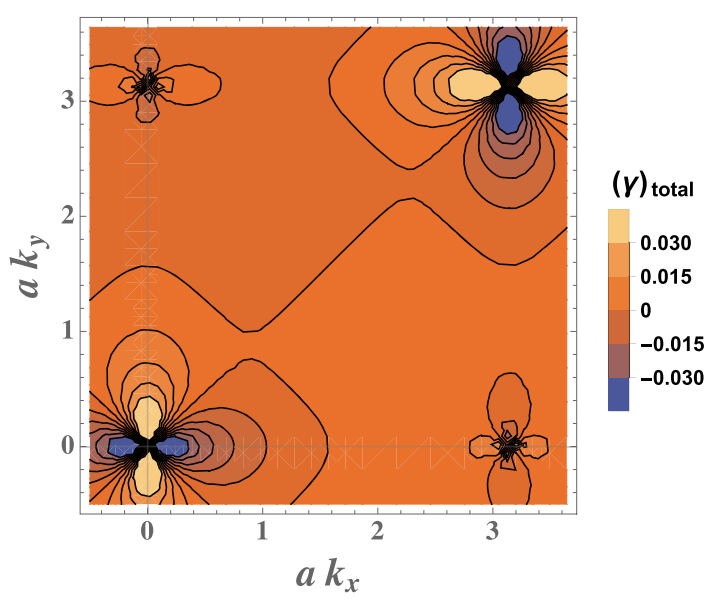

FIG. 4. Contour plot of the density $\gamma_{\text {total }}(\mathbf{k})$ for the $C_{2}$ symmetric Chern insulator. The plot is shown with the hopping parameters $t=20$ and $t^{\prime}=21, m=0.25, a=1$, and $r=1$. 
majority of the variation in the density is captured by the quadrupolar lobes near each of the Dirac points, whose contributions integrate to zero. Beyond the momentum cutoff, this cancellation is imperfect, and we recover the nonvanishing of $\gamma_{\text {total }}$ shown in Fig. 3.

To summarize, we have found that in the continuum limit, the anisotropic viscosities of the Chern insulator vanish just as they do for a $C_{2}$-anisotropic Dirac theory in the continuum.

\section{Anisotropic superfluids}

Paired superfluids, which spontaneously break parity and time-reversal symmetry in the superfluid phase, also naturally realize a Hall viscosity. If we include anisotropy in the system, these superfluids can have nonzero values for the viscosity coefficients in Eq. (76). The general meanfield Hamiltonian we work with is given by [60]

$$
\begin{aligned}
H= & \int d^{2} x \psi^{\dagger}(\mathbf{x})\left(-\frac{1}{2 m} \mathcal{E}^{i j} \partial_{i} \partial_{j}-\mu\right) \psi(\mathbf{x}) \\
& +\frac{1}{2} \int d^{2} x^{\prime} \int d^{2} x\left[\Delta\left(\mathbf{x}-\mathbf{x}^{\prime}\right) \psi^{\dagger}(\mathbf{x}) \psi^{\dagger}\left(\mathbf{x}^{\prime}\right)+\text { H.c. }\right] .
\end{aligned}
$$

The rotational symmetry group of this Hamiltonian depends on the symmetric mass tensor $\mathcal{E}^{i j}$. We can write (defining parameters $\alpha$ and $\beta$ for the parity even and odd anisotropic perturbations, respectively)

$$
\mathcal{E}_{i j}=\delta_{i j}+\kappa\left\{\beta \sigma_{i j}^{x}+\alpha \sigma_{i j}^{z}\right\} .
$$

To break time-reversal symmetry, we require a complex gap function, and we consider a paired $\ell$-wave complex superfluid, with gap function

$$
\Delta_{\mathbf{k}}=\left|\Delta_{\mathbf{k}}\right| e^{i \ell \phi} .
$$

This form of the gap breaks both parity and time reversal but is even under the composite symmetry $P T$, whereas the kinetic term in the Hamiltonian is only $P T$ even when $\beta=0$. We can utilize a Bogoliubov transformation to find the quasiparticle excitation spectrum. In this basis, the Hamiltonian takes the form

$$
H=\sum_{\mathbf{k}} E_{\mathbf{k}} d_{\mathbf{k}}^{\dagger} d_{\mathbf{k}}+E_{0} .
$$

The transformation is

$$
\begin{aligned}
& c_{\mathbf{k}}=u_{\mathbf{k}}^{*} d_{\mathbf{k}}-v_{-\mathbf{k}} d_{-\mathbf{k}}^{\dagger}, \\
& c_{\mathbf{k}}^{\dagger}=u_{\mathbf{k}} d_{\mathbf{k}}^{\dagger}-v_{-\mathbf{k}}^{*} d_{-\mathbf{k}} .
\end{aligned}
$$

There is a redundancy for the functions $u_{\mathbf{k}}, v_{\mathbf{k}}$, and we can choose a gauge in which $u_{\mathbf{k}}$ is real. In this gauge, we have that

$$
v_{\mathbf{k}}=\left|v_{\mathbf{k}}\right| e^{i \ell \phi}
$$

transforms as an $\ell$ pole under rotations. Its magnitude is given by [60]

$$
\left|v_{\mathbf{k}}\right|^{2}=\frac{1}{2}\left(1-\frac{\epsilon_{\mathbf{k}}}{E_{\mathbf{k}}}\right)
$$

In this gauge, $v_{\mathbf{k}}$ transforms in the same way under rotations as the gap $\Delta_{\mathbf{k}}$. The dispersion relation for the Hamiltonian (214) is given by

$$
\begin{aligned}
E_{\mathbf{k}} & =\sqrt{\left(\frac{1}{2 m}\left(\mathcal{E}^{i j} k_{i} k_{j}\right)-\mu\right)^{2}+\left|\Delta_{\mathbf{k}}\right|^{2}} \\
& \equiv \sqrt{\epsilon_{\mathbf{k}}^{2}+\left|\Delta_{\mathbf{k}}\right|^{2}}
\end{aligned}
$$

To compute the viscosity for this model, we use the fact that there are no internal degrees of freedom, so we can make use of the formalism of Ref. [37] directly. Integrating the Kubo formula (70) by parts, we can use the strain-strain form of the viscosity,

$$
\eta_{\nu}^{\mu \lambda}{ }_{\rho}^{\lambda}(\omega \rightarrow 0)=-i\left\langle\left[J_{\nu}^{\mu}, J_{\rho}^{\lambda}\right]\right\rangle_{0} .
$$

This form is odd under time reversal, so the full viscosity is a Hall viscosity at zero frequency. In terms of quasiparticle operators, the ground-state expectation value of the strain operators is, from Refs. [6,37],

$\left\langle J^{\mu}{ }_{\nu}\right\rangle_{0}=\frac{-i L^{2}}{4} \int \frac{d^{2} k}{(2 \pi)^{2}} k_{\nu}\left(v_{\mathbf{k}} \partial_{k_{\mu}} v_{\mathbf{k}}^{*}-v_{\mathbf{k}}^{*} \partial_{k_{\mu}} v_{\mathbf{k}}\right)$.

Using the commutation relation between strain generators,

$$
\left[J_{\nu}^{\mu}, J_{\rho}^{\lambda}\right]=-i\left(\delta_{\rho}^{\mu} J_{\nu}^{\lambda}-\delta_{\nu}^{\lambda} J_{\rho}^{\mu}\right)
$$

We can write the zero-frequency viscosity for the superfluid as

$$
\left(\eta^{\mathrm{H}}\right)^{\mu}{ }_{\nu \rho}^{\lambda}=\delta_{\nu}^{\lambda}\left\langle\mathrm{J}_{\rho}^{\mu}\right\rangle_{0}-\delta_{\rho}^{\mu}\left\langle\mathrm{J}^{\lambda}{ }_{\nu}\right\rangle_{0} .
$$

We find that the independent components of the groundstate strain give exactly the three traceless components of the Hall viscosity; hence, we can decompose the strain expectation into irreducible representations as

$$
\left\langle J^{\mu}{ }_{\nu}\right\rangle_{0}=\eta^{\mathrm{H}} \epsilon^{\mu}{ }_{\nu}+\gamma\left(\sigma^{x}\right)^{\mu}{ }_{\nu}-\Theta\left(\sigma^{z}\right)^{\mu}{ }_{\nu} .
$$

The viscosity coefficients are then given by 


$$
\begin{aligned}
\eta^{\mathrm{H}} / L^{2} & =\frac{l}{4} \int \frac{d^{2} k}{(2 \pi)^{2}}\left|v_{\mathbf{k}}\right|^{2}, \\
\gamma / L^{2} & =-\frac{l}{4} \int \frac{d^{2} k}{(2 \pi)^{2}} \cos (2 \phi)\left|v_{\mathbf{k}}\right|^{2}, \\
\Theta / L^{2} & =-\frac{l}{4} \int \frac{d^{2} k}{(2 \pi)^{2}} \sin (2 \phi)\left|v_{\mathbf{k}}\right|^{2} .
\end{aligned}
$$

We can see the anisotropy of the anomalous coefficients explicitly, and it is clear that they vanish if the dispersion $\epsilon_{\mathbf{k}}$ is isotropic. To lowest order in the small parameter $\kappa$,

$$
\begin{aligned}
\eta^{\mathrm{H}} & =\frac{1}{2} \bar{n} \bar{s}, \\
\gamma & =\frac{l}{32} \kappa \alpha \mathcal{I}, \\
\Theta & =\frac{l}{32} \kappa \beta \mathcal{I} .
\end{aligned}
$$

The isotropic Hall viscosity is quantized as in Ref. [37] by the mean particle number density and angular momentum per particle, while the anomalous coefficients enter at first order in the anisotropy and both depend on the integral

$$
\mathcal{I}=\frac{L^{2}}{(2 \pi)^{2}} \int d k \frac{\pi k^{3}\left|\Delta_{\mathbf{k}}\right|^{2}}{m E_{\mathbf{k} 0}^{3}}
$$

where $E_{\mathbf{k} 0}=\lim _{\kappa \rightarrow 0} E_{\mathbf{k}}$. This result is consistent with the results of Ref. [55].

\section{EXPERIMENTAL IMPLICATIONS}

A large motivation for our study is experimental, especially as the Hall viscosity has seen a bevy of theoretical proposals for measurement and an initial positive measurement in graphene. We see our work as an opportunity to extend the opportunities for this developing field of the experimental study of quantum fluids without time-reversal symmetry. Our lattice formalism gives a starting point for researchers looking at hydrodynamic and ballistic regimes in quantum materials and, importantly, can shed light on whether anisotropic corrections to $\mathbf{k} \cdot \mathbf{p}$ theories, often invoked in materials like graphene, are important. In particular, in materials where "warping terms" - nonrotationally invariant contributions to continuum Hamiltonians - are important, considering anomalous Hall viscosity components is necessary, as we have done in this work. In this section, we comment on the experimental implications of our formalism.

First, in Sec. VII A, we present an anisotropic generalization of the width-dependent corrections to the resistivity in the hydrodynamic regime $[17,19]$, which are experimentally accessible. Second, following the framework of Ref. [21], we remark on implications of our anisotropic viscosity for nonhydrodynamic transport in Sec. VII B.
Third, in Sec. VII C, we argue that Hall viscosity may be detectable in future torque magnetometry experiments in clean two-dimensional systems. Finally, in Sec. VII D, we remark on new material platforms in which our formalism for anisotropic and lattice viscosity may yield new probes for topological physics beyond clean, noninteracting electron systems.

\section{A. Anisotropic pipe flow: The (viscous) Hall bar}

In relaxing the assumption of isotropy, we find six independent viscosities that provide three viscous forces. Generalizing the approach of Ref. [17], we can consider the classical viscous Hall effect in a rectangular Hall bar sample with width $W$ along the $y$ direction and obtain viscous corrections to the classical Hall resistivity. For our set of viscous forces dictated by the tensor (78), we have three distinct viscous corrections to the resistivity, two of them being anisotropic. If we orient our Cartesian axes at an angle $\alpha$ with respect to the $C_{2}$ axes of our sample, we find that the change in resistivity from the classical value $\Delta \rho_{x y}=\rho_{x y}-\rho_{x y}^{\text {bulk }}$, with $\rho_{x y}^{\text {bulk }}=-\left(m \omega_{c} / e^{2} n\right)$, is given by

$$
\begin{aligned}
\Delta \rho_{x y}= & -\frac{\rho_{x y}^{\text {bulk }}}{A \omega_{c}} \frac{12}{W^{2}-12 l_{s}^{2}} \\
& \times\left[\left(\eta^{H}\right)_{\text {total }}-\gamma_{\text {total }} \cos (2 \alpha)-\Theta_{\text {total }} \sin (2 \alpha)\right] .
\end{aligned}
$$

Above, we used partial slip boundary conditions with slip length $l_{s}$ [19]. Our result differs by a factor of area $A=L W$ since Ref. [17] works with kinematic rather than dynamic viscosities. We can thus pick out the different anisotropic contributions to the viscosity by modifying the sample angle $\alpha$ with respect to the $C_{2}$ axes. The effect of the total anisotropic viscosities $\gamma_{\text {total }}$ and $\Theta_{\text {total }}$ can thus be experimentally probed by varying the orientation of the pipe relative to the sample. This setup could be used to probe both lattice anisotropy and nematicity, for instance, in anisotropic quantum Hall states at $\nu=7 / 3$ [61].

For lattice systems, the formalism of Sec. III allows us to explore momentum transport beyond the hydrodynamic regime as well. In particular, by quantifying the effect of Umklapp scattering on momentum relaxation in Eq. (54), our formalism allows for a quantitative exploration of the crossover between diffusive and hydrodynamic transport. This domain can be accessed experimentally in systems with tunable disorder such as incommensurate TMD heterostructures.

Finally, implicit in all pipe-flow predictions is a choice of boundary conditions at the edge of the pipe, such as our partial-slip boundary conditions above. Different boundary conditions on the tangential and normal stresses can be reinterpreted as bulk divergenceless contributions to the stress tensor and hence correspond to different choices for the splitting between barred and unbarred viscosity coefficients. This case has recently been explored (albeit not 
with this perspective) in the propagation of surface waves in isotropic chiral fluids [62-65]. Using our formalism, the redundancy in nondissipative (and dissipative) viscosities can be explored quantitatively through wave propagation at the surface of classical chiral active fluids [66] as well as through the boundary behavior of currents in pipe flow experiments and fluid flow around obstacles.

\section{B. Beyond hydrodynamic flow}

Following Ref. [21], we can study the Hall viscosity in anisotropic systems in the semiclassical regime. Reference [21] establishes a relationship between the experimentally measurable angular moments of the semiclassical distribution function and the Hall viscosity in the isotropic case, where those moments can be expanded in terms of angular harmonics. An extension of this treatment to the anisotropic case promises to yield a relationship between the now more complicated moments of the distribution function and our anisotropic Hall viscosities, effectively generalizing the relationship between transverse electric field response and Hall viscosity. Such relationships are valid even beyond the hydrodynamic regime and are therefore of great experimental interest.

\section{Torque magnetometry}

Another property of quantum fluids without timereversal symmetry that we can probe is magnetization. In the presence of a nonuniform electric field, there is a viscous response that gives rise to a momentum-dependent contribution to the Hall conductivity [37]. The momentumdependent current density that arises as a result will give rise to a manifestly viscous contribution to the bulk magnetization of the sample, one that we can directly couple to the "total" nondissipative Hall viscosities in Eq. (83) and measure via torque magnetometry.

In particular, the magnetization density

$$
\delta M=\frac{1}{2} \int d^{2} r(\mathbf{r} \times \mathbf{j})_{z}
$$

receives contributions from the wave-vector-dependent conductivity and hence from the Hall viscosity. In a $C_{2}$ invariant system, one can analyze the different types of viscous magnetization responses, noting that the anisotropic viscosities will produce magnetizations that transform nontrivially under rotations, i.e., in-plane multipole moments. Torque magnetometry would be able to probe these higher-moment responses [67-69].

\section{Viscosity as a probe of new material systems}

There are a host of materials that could provide a domain for realizing the predictions in this work. Black phosphorus is one example of an anisotropic material that supports hydrodynamic flow [70,71]. Furthermore, $\mathrm{PdCoO}_{2}$ is another material where electron viscosity plays a role in transport and has a hexagonal Fermi surface [72]. In both of these systems, the anisotropic Hall viscosity can be used as a probe of the interplay between Berry curvature and intra-unit-cell angular momentum, following our results in Sec. V.

Beyond free-fermion systems, our formalism has more general implications for topological physics in strongly correlated systems in a magnetic field, such as fractional quantum Hall states in twisted bilayer graphene and graphene-boron nitride moiré heterostructures [73-75]. At the fundamental level, our formalism allows for the identification of the Belinfante stress tensor in models of these systems, both with and without a magnetic field. Going further, the rich emergent symmetry structure in fractional quantum Hall and fractional Chern insulator states in these materials could allow for the evaluation of the anisotropic Hall viscosity, similar to Refs. [6,7]. As graphene-based Chern insulators are both anisotropic and amenable to hydrodynamic measurements [22,76], our formalism could thus enable the experimental investigation of the interplay between geometry and topology in these systems. Finally, cold-atom systems with artificial gauge fields can be used to realize the Hofstadter model on a variety of lattices, both with and without interactions $[77,78]$. These systems are a promising platform to investigate the experimental implications of our lattice formulation of momentum transport.

\section{OUTLOOK}

In this work, we have extended the Kubo formalism for viscosity to systems with rotational-symmetry-breaking anisotropy, discrete translational symmetry, and internal rotational degrees of freedom. At the microscopic level, we have shown that the symmetrization procedure for the stress tensor given in Ref. [36] is equivalent to the generalized Belinfante procedure in nonrelativistic field theory, which involves restricting ourselves to strain perturbations that do not change the torsion of space. Furthermore, we have argued that this choice for the stress tensor applies to systems without rotational symmetry as well. We have shown that in systems with discrete translational symmetry, it is possible to define a course-grained momentum density and stress tensor and hence compute viscoelastic response functions. We also argued that our coarse-grained lattice formalism reduces to the continuum formalism in the hydrodynamic limit.

More generally, we have also analyzed the symmetry of the viscosity tensor, highlighting the importance of the six independent Hall viscosity coefficients. We have shown that only three linear combinations of these coefficients enter into the hydrodynamic equations of motion in the bulk. The other three linear combinations can be changed by adding divergenceless terms to the viscous stress tensor, and hence they have no measurable effects on the absence 
of a physical principle (such as gravity or boundary conditions) fixing the divergenceless part of the stress tensor. We show that the three linear combinations that determine the forces can be assembled into a rank-two "Hall tensor," which coincides with the tensor first highlighted by Haldane in Ref. [51]. Finally, we applied our formalism to a variety of free-fermion systems, both in the continuum and on the lattice. We showed that free fermions in the continuum always have vanishing Hall elastic moduli, consistent with expectations for a fluid. We also showed that the six viscosity coefficients can be expressed in terms of quadrupole moments of the Berry curvature and matrix elements of the internal angular-momentum operator. When focusing on the Hall tensor (i.e., the viscous forces), we found that the Berry curvature contribution to the viscous forces canceled, and only the internal angular momentum contributed. We showed how our formalism works in practice by analyzing an anisotropic Dirac fermion. We performed a similar analysis for free fermions on the lattice and compared our results for the lattice regularized Dirac fermion (Chern insulator) with those of Ref. [30]. Crucially, we found within our formalism that the low-energy expansion of the Hall tensor in the Chern insulator coincides with the Hall tensor for four isolated Dirac fermions in the continuum, representing a consistency check on the formalism. Lastly, we showed how our formalism applies to anisotropic superfluids, obtaining results consistent with the symmetry analysis of Ref. [55].

Going forward, our results raise several important questions for the Hall viscosity of time-reversal symmetry-broken anisotropic systems. First, our emphasis on viscous forces and the Hall tensor has direct relevance for experiments geared to extract the viscosity from hydrodynamic flow. As we argued, in a threefold or higher symmetric system, an analysis of flow can only extract the combination $\eta^{\mathrm{H}}+\bar{\eta}^{\mathrm{H}}=\eta_{\text {total }}^{\mathrm{H}}$ that enters the Hall tensor. This fact has been overlooked in the current theoretical analysis of flow in graphene and other materials, which assume rotational symmetry and fixed boundary conditions. We sketched the experimental implications of this in Sec. VII A. Additionally, we have shown in Sec. VII C that our results have implications for torque magnetometry experiments on anisotropic quantum Hall systems. Furthermore, our formalism can be used to explain current and future experiments on viscous transport in anisotropic lattice systems such as twisted bilayer graphene, black phosphorus, and fractional Chern insulators in grapheneboron nitride heterostructrues.

For free-fermion systems, we have seen that working with only the kinetic stress leads to $\eta_{\text {total }}^{\mathrm{H}}=0$. This result implies that for free fermions, the "torsional viscosity" computed with the spin connection set to zero (i.e., using only the kinetic stress) does not enter into the equations of motion for momentum transport. Furthermore, our lattice formalism suggests that the derivation of the stress tensor in low-energy theories of Bloch electrons should be revisited. The momentum dependence of the lattice strain generators (51) suggests that the displacement of Fermi-surface pockets away from zero momentum (as in graphene) may have a significant effect on viscous response functions. Additionally, the lattice formulation of momentum transport will allow for the exploration of the crossover between ballistic and diffusive transport in lattice models of quantum systems. Another interesting direction for future work is to extend our formalism to three-dimensional systems such as topological semimetals [79-81]. Furthermore, the redundancy (84) in the dissipative and nondissipative viscous force densities could have interesting implications for hydrodynamic waves and entropy production in anisotropic fluids. Finally, there appears to be some connection to explore between our formulation of lattice momentum transport and the Kähler-Dirac formalism of lattice gauge theory [82].

\section{ACKNOWLEDGMENTS}

B. B. would like to thank F. D. M. Haldane and N. Read for conversations that inspired this work. The authors also thank J. Schmalian for pointing out the crucial Ref. [36]. Additionally, the authors acknowledge fruitful discussions with A. Abanov, E. Fradkin, T. L. Hughes, D. Huse, and M. Stone. This material is based upon work supported by the National Science Foundation under Grant No. DGE 1746047.

\section{APPENDIX: ALTERNATIVE FORM OF THE LATTICE SPIN STRESS}

Reexamining Eq. (51) for the strain generators on the lattice, we note that another convenient choice of spin strain would be

$$
\begin{aligned}
\mathcal{J}_{\mathrm{L}, \nu}^{\mu} \equiv & -\frac{i}{2} \sum_{\mathbf{k} n m \nu} c_{n \mathbf{k}}^{\dagger}\left[\left\{\frac{\sin \mathbf{k} \cdot \mathbf{a}_{\nu}}{\left|\mathbf{a}_{\nu}\right|}, \frac{\partial}{\partial k_{\mu}}\right\} \delta^{n m}\right. \\
& \left.+i \tilde{\epsilon}_{\nu}{ }^{\mu} \cos \mathbf{k} \cdot \mathbf{a}_{\nu} L_{\text {int }}^{n m}\right] c_{m \mathbf{k}},
\end{aligned}
$$

which differs from Eq. (51) in the index of the cosine term in the spin stress. The convenience of this term becomes manifest when we compute the free-fermion stress tensor

$$
\begin{aligned}
T_{\mathrm{B} 0, \nu}^{\mu}= & \sum_{n m \mathbf{k}} c_{n \mathbf{k}}^{\dagger}\left(\partial^{\mu} f^{n m}(\mathbf{k}) \frac{\sin \mathbf{k} \cdot \mathbf{a}_{\nu}}{\left|\mathbf{a}_{\nu}\right|}\right. \\
& \left.+\frac{i}{2} \tilde{\epsilon}^{\mu}{ }_{\nu} \cos \mathbf{k} \cdot \mathbf{a}_{\nu}\left[f(\mathbf{k}), L_{\mathrm{int}}\right]_{n m}\right) c_{m \mathbf{k}} \\
= & \sum_{n m \mathbf{k}} c_{n \mathbf{k}}^{\dagger}\left[T_{\mathrm{B} \rho}^{\lambda}(\mathbf{k})\right]^{n m} c_{m \mathbf{k}},
\end{aligned}
$$

which follows from this definition. We now see that the index of $\mathbf{k}$ in the cosine prefactor of the spin stress matches 
the second, lower index on the stress tensor. If we were to expand this new lattice stress about the time-reversal invariant momenta in the Brillouin zone of a crystal, we would find that the overall sign of the spin-stress obtained from Taylor expanding the cosine term would match the overall sign of the kinetic stress obtained from expanding the sine in the first term. While this gives the same result as our Eq. $(57)$ at the $(0,0)$ and $(\pi, \pi)$ points, it differs at the $(0, \pi)$ and $(\pi, 0)$ points.

At first glance, the extra semblance of rotational symmetry gained by this change of index may seem appealing. However, we can ask what form the internal momentum density must take to produce this term in the strain generator. It can be shown that Eq. (A1) implies

$$
\begin{aligned}
g_{\mu}^{\mathrm{int}}(\mathbf{R})= & \frac{1}{4\left|\mathbf{a}_{\nu}\right|} \tilde{\epsilon}_{\mu} \sum_{n m} L_{\mathrm{int}}^{n m}\left(c_{n \mathbf{R}+\mathbf{a}_{\nu}+\mathbf{a}_{\mu}}^{\dagger} c_{m \mathbf{R}+a_{\mu}}-c_{n \mathbf{R}}^{\dagger} c_{m \mathbf{R}+a_{\mu}}\right. \\
& \left.+c_{n \mathbf{R}+\mathbf{a}_{\nu}}^{\dagger} c_{m \mathbf{R}+\mathbf{a}_{\nu}+\mathbf{a}_{\mu}}-c_{n \mathbf{R}+\mathbf{a}_{\mu}}^{\dagger} c_{m \mathbf{R}}\right) .
\end{aligned}
$$

This discretization of the lattice derivative has the unfortunate features of not being symmetric with respect to the base point of differentiation and of not including all nearest-neighbor lattice sites to which momentum can flow. For these reasons, we know of no justification for using Eq. (A3) in place of Eq. (41).

Nevertheless, we can explore the consequences of Eq. (A3) on the viscosity of the Chern insulator model considered in Sec. VI B. For the modified stress tensor, we find

$$
\begin{aligned}
T_{\mathrm{B} \rho}^{\lambda}(\mathbf{k})= & t^{\lambda} \cos \left(k_{\lambda} a\right) \sin \left(k_{\rho} a\right) \sigma^{\lambda}+r \sin \left(k_{\lambda} a\right) \sin \left(k_{\rho} a\right) \sigma^{z} \\
& +\frac{1}{2} \cos \left(k_{\rho} a\right)\left(t^{\lambda} \sin \left(k_{\lambda} a\right) \sigma^{\rho}-t^{\rho} \sin \left(k_{\rho} a\right) \sigma^{\lambda}\right) .
\end{aligned}
$$

The spin stress tensor here differs from Eq. (194) by a factor of $\cos k_{\rho} a / \cos k_{\lambda} a$.

$C_{4}$ case.-For a $C_{4}$-symmetric system with hoppings $t=t^{\prime}$, this case leads to a total viscosity that is now simple enough to present,

$$
\begin{aligned}
\eta_{\text {total }}^{\mathrm{H}}(\mathbf{k})= & \frac{t^{2}}{32 \epsilon_{\mathbf{k}}^{3}}\left\{r \cos \left(k_{y} a\right)+\cos \left(k_{x} a\right)\left[r-m \cos \left(k_{y} a\right)\right]\right. \\
& \left.\times\left[\cos \left(2 k_{x} a\right)+\cos \left(2 k_{y} a\right)-2\right]\right\} .
\end{aligned}
$$

The $C_{4}$-symmetric viscosities are plotted in Fig. 5. The expansion of the Hamiltonian around each of the Dirac points is the same as in Eq. (202). The expansion of this tensor around the Dirac points can be written as

$$
\begin{aligned}
{\left[T_{\mathrm{B}, \rho}^{\lambda}(\mathbf{k})\right]^{(i)} } & =v_{F}\left[\alpha_{\lambda}^{i} \alpha_{\rho}^{i} \sigma^{\lambda} k_{\rho}+\frac{1}{2} \alpha_{\rho}^{i}\left(\alpha_{\lambda}^{i} k_{\lambda} \sigma^{\rho}-\alpha_{\rho}^{i} k_{\rho} \sigma^{\lambda}\right)\right] \\
& =\frac{v_{F}}{2}\left[\left(2 \alpha_{\lambda}^{i}-\alpha_{\rho}^{i}\right) \alpha_{\rho}^{i} \sigma^{\lambda} k_{\rho}+\alpha_{\rho}^{i} \alpha_{\lambda}^{i} k_{\lambda} \sigma^{\rho}\right] .
\end{aligned}
$$

The resulting viscosity can be computed, and it takes a similar Dirac-point-dependent form as Eq. (205). The sum

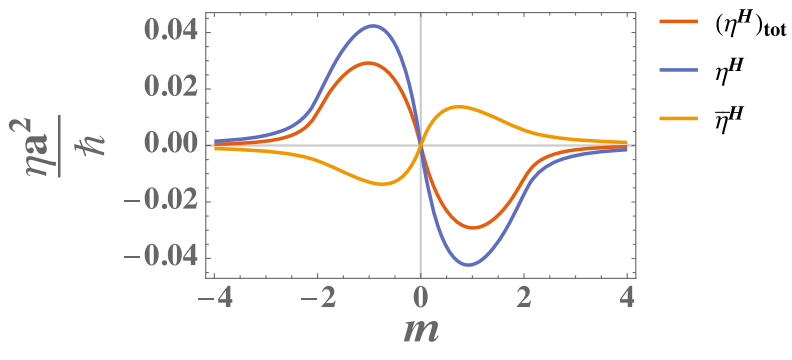

FIG. 5. Hall viscosities in $C_{4}, \eta_{\text {total }}^{\mathrm{H}}, \eta^{\mathrm{H}}, \bar{\eta}^{H}$, plotted with the alternative form of the strain generator, with parameters $t=t^{\prime}=$ $r=1$ and lattice constant $a=1$. We see much less variation in the plots in this case, while the same features of oddness in $m$ and vanishing in the topologically trivial regimes remain.

over Dirac points now yields the same expression by magnitude, but this result now changes sign across the Dirac points:

$\left[\eta_{\text {total }}^{H}(\mathbf{k})\right]^{(i)}=\left[\eta^{\mathrm{H}}(\mathbf{k})+\bar{\eta}^{H}(\mathbf{k})\right]^{(i)}=\alpha_{x}^{i} \alpha_{y}^{i} \frac{v_{F}^{2} M^{i}}{8 \epsilon_{\mathbf{k}}^{3}}|\mathbf{k}|^{2}$,

which has the same form as the isotropic Hall viscosity in Ref. [30]. Integrating this up to a momentum cutoff $\Lambda$ and summing over the Dirac points yields the cutoffindependent viscosity

$$
\begin{aligned}
\eta_{\text {total }}^{H} & =-\frac{1}{2} \sum_{i} \frac{\left(\alpha_{x}^{i} \alpha_{y}^{i}\right)}{4 \pi v_{F}^{2}}\left[M^{i}\left|M^{i}\right|\right] \\
& =\frac{1}{2} \begin{cases}\frac{\hbar}{2 \pi v_{F}^{2}}(-m|m|+4 m r) & |m|<2 r \\
\frac{2 \hbar}{\pi v_{F}^{2}} r^{2} & |m| \geq 2 r .\end{cases}
\end{aligned}
$$

We find that the cutoff-independent total viscosity obtained with this alternative Belinfante procedure coincides with the cutoff-independent kinetic viscosity computed in Ref. [30].

Again separating into the individual barred and unbarred Hall viscosities separately, we see that the end points $(0,0)$ and $(\pi, \pi)$ are described by fully isotropic theories, meaning the unbarred Hall viscosity is the only nonzero contribution $\left(\eta_{\mathrm{total}}^{\mathrm{H}}\right)^{(0,3)}=\eta^{\mathrm{H}}$. However, at the corner points $(\pi, 0)$ and $(0, \pi)$,

$$
\begin{aligned}
\eta^{\mathrm{H}}(\mathbf{k}) & =\frac{-v_{F}^{2} m k^{2}}{4 \epsilon_{\mathbf{k}}^{3}}, \\
\bar{\eta}^{H} & =\frac{v_{F}^{2} m k^{2}}{8 \epsilon_{\mathbf{k}}^{3}} .
\end{aligned}
$$

$\mathrm{C}_{2}$ case.-We see a similar picture in the general case. The anisotropic viscosities $\gamma_{\text {total }}$ are plotted in Fig. 6. We note that for this alternative choice of stress tensor, the total anisotropic viscosity is identically zero. 


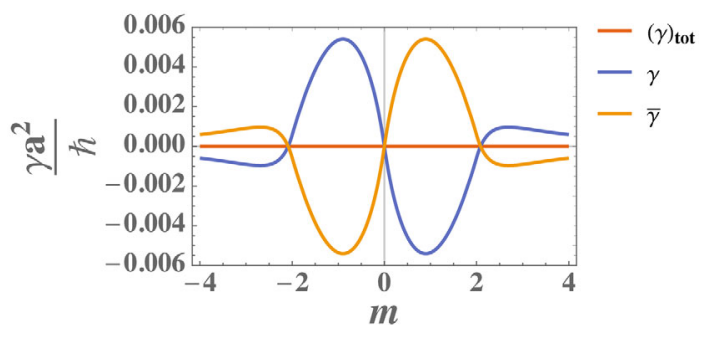

FIG. 6. Anisotropic viscosities plotted with the alternative form of the strain generator. We use the same set of parameters $t=1.5$, $t=r=1, a=1$ as earlier in the work. Curiously, we see that the total anisotropic viscosity is zero.

The continuum expansion is also cutoff independent with this choice of strain generator, as we see below:

$$
\begin{aligned}
\left(\eta_{\text {total }}^{\mathrm{H}}\right)^{(i)} & =-\frac{1}{2} \sum_{i} \frac{\left(\alpha_{x}^{i} \alpha_{y}^{i}\right)}{4 \pi\left\langle v_{F}\right\rangle_{g}^{2}}\left[M^{i}\left|M^{i}\right|\right] \\
& =\frac{1}{2} \begin{cases}\frac{\hbar}{2 \pi\left\langle v_{F}\right\rangle_{g}^{2}}(-m|m|+4 m r) & |m|<2 r \\
\frac{2 \hbar}{\pi\left\langle v_{F}\right\rangle_{g}^{2}} r^{2} & |m| \geq 2 r .\end{cases}
\end{aligned}
$$

The anisotropic viscosities are independent of this choice of strain generator [independent of the $\cos \left(k_{\lambda} a\right)$ vs $\cos \left(k_{\rho} a\right)$ prefactor in $\left(T^{\text {spin }}\right)_{\rho}^{\lambda}$ ]-they are zero.

[1] K. v. Klitzing, G. Dorda, and M. Pepper, New Method for High-Accuracy Determination of the Fine-Structure Constant Based on Quantized Hall Resistance, Phys. Rev. Lett. 45, 494 (1980).

[2] R. B. Laughlin, Quantized Hall Conductivity in Two Dimensions, Phys. Rev. B 23, 5632 (1981).

[3] D. J. Thouless, M. Kohmoto, M. P. Nightingale, and M. den Nijs, Quantized Hall Conductance in a Two-Dimensional Periodic Potential, Phys. Rev. Lett. 49, 405 (1982).

[4] J.E. Avron, R. Seiler, and P. G. Zograf, Viscosity of Quantum Hall Fluids, Phys. Rev. Lett. 75, 697 (1995).

[5] I. V. Tokatly and G. Vignale, Lorentz, Shear Modulus of a Two-Dimensional Electron Gas at High Magnetic Field, Phys. Rev. B 76, 161305(R) (2007).

[6] N. Read, Non-Abelian Adiabatic Statistics and Hall Viscosity in Quantum Hall States and $p_{x}+i p_{y}$ Paired Superfluids, Phys. Rev. B 79, 045308 (2009).

[7] N. Read and E. H. Rezayi, Hall Viscosity, Orbital Spin, and Geometry: Paired Superfluids and Quantum Hall Systems, Phys. Rev. B 84, 085316 (2011).

[8] F. D. M. Haldane, Fractional Quantization of the Hall Effect: A Hierarchy of Incompressible Quantum Fluid States, Phys. Rev. Lett. 51, 605 (1983).

[9] M. P. Zaletel, R. S. K. Mong, and F. Pollmann, Topological Characterization of Fractional Quantum Hall Ground States from Microscopic Hamiltonians, Phys. Rev. Lett. 110, 236801 (2013).
[10] F. D. M. Haldane and Yu. Shen, "Geometry of Landau Orbits in the Absence of Rotational Symmetry," arXiv:1512.04502.

[11] A. Gromov, S. D. Geraedts, and B. Bradlyn, Investigating Anisotropic Quantum Hall States with Bimetric Geometry, Phys. Rev. Lett. 119, 146602 (2017).

[12] B. Offertaler and B. Bradlyn, Viscoelastic Response of Quantum Hall Fluids in a Tilted Field, Phys. Rev. B 99, 035427 (2019).

[13] A. Souslov, A. Gromov, and V. Vitelli, Anisotropic Odd Viscosity via Time-Modulated Drive, arXiv:1909.08505.

[14] R. Karplus and J. M. Luttinger, Hall Effect in Ferromagnetics, Phys. Rev. 95, 1154 (1954).

[15] F. D. M. Haldane, Berry Curvature on the Fermi Surface: Anomalous Hall Effect as a Topological Fermi-Liquid Property, Phys. Rev. Lett. 93, 206602 (2004).

[16] A. Lucas and K. C. Fong, Hydrodynamics of Electrons in Graphene, J. Phys. Condens. Matter 30, 053001 (2018).

[17] T. Scaffidi, N. Nandi, B. Schmidt, A. P. Mackenzie, and J. E. Moore, Hydrodynamic Electron Flow and Hall Viscosity, Phys. Rev. Lett. 118, 226601 (2017).

[18] P. S. Alekseev, Negative Magnetoresistance in Viscous Flow of Two-Dimensional Electrons, Phys. Rev. Lett. 117, 166601 (2016).

[19] L. V. Delacrétaz and A. Gromov, Transport Signatures of the Hall Viscosity, Phys. Rev. Lett. 119, 226602 (2017).

[20] F. M. D. Pellegrino, I. Torre, and M. Polini, Nonlocal Transport and the Hall Viscosity of Two-Dimensional Hydrodynamic Electron Liquids, Phys. Rev. B 96, 195401 (2017).

[21] T. Holder, R. Queiroz, and A. Stern, Unified Description of the Classical Hall Viscosity, Phys. Rev. Lett. 123, 106801 (2019).

[22] A. I. Berdyugin, S. G. Xu, F. M. D. Pellegrino, R. K. Kumar, A. Principi, I. Torre, M. B. Shalom, T. Taniguchi, K. Watanabe, I. V. Grigorieva et al., Measuring Hall Viscosity of Graphenes Electron Fluid, Science 364, 162 (2019).

[23] B. N. Narozhny and M. Schütt, Magnetohydrodynamics in Graphene: Shear and Hall Viscosities, Phys. Rev. B 100, 035125 (2019).

[24] M. Imran, Quantizing Momentum Transport in Bilayer Graphene, arXiv:1909.09608.

[25] B. N. Narozhny, Electronic Hydrodynamics in Graphene, Ann. Phys. (Amsterdam) 411, 167979 (2019).

[26] D. T. Son, Chiral Metric Hydrodynamics, Kelvin Circulation Theorem, and the Fractional Quantum Hall Effect, arXiv:1907.07187.

[27] S. Pu, M. Fremling, and J. K. Jain, Hall Viscosity of Composite Fermions, Phys. Rev. Research 2, 013139 (2020).

[28] A. Buchel and M. Baggioli, Holographic Viscoelastic Hydrodynamics, J. High Energy Phys. 03 (2019) 146.

[29] S. S. Apostolov, D. A. Pesin, and A. Levchenko, Magnetodrag in the Hydrodynamic Regime: Effects of Magnetoplasmon Resonance and Hall Viscosity, Phys. Rev. B 100, 115401 (2019).

[30] H. Shapourian, T. L. Hughes, and S. Ryu, Viscoelastic Response of Topological Tight-Binding Models in Two and Three Dimensions, Phys. Rev. B 92, 165131 (2015).

[31] T. I. Tuegel and T. L. Hughes, Hall Viscosity and Momentum Transport in Lattice and Continuum Models of the 
Integer Quantum Hall Effect in Strong Magnetic Fields, Phys. Rev. B 92, 165127 (2015).

[32] L. Dong and Q. Niu, Geometrodynamics of Electrons in a Crystal under Position and Time-Dependent Deformation, Phys. Rev. B 98, 115162 (2018).

[33] I. S. Burmistrov, M. Goldstein, M. Kot, V. D. Kurilovich, and P. D. Kurilovich, Dissipative and Hall Viscosity of a Disordered 2D Electron Gas, Phys. Rev. Lett. 123, 026804 (2019).

[34] L. D. Landau and E. M. Lifshitz, Fluid Mechanics, 2nd ed. (Pergamon, Oxford, 1987).

[35] B. Bradlyn and N. Read, Low-Energy Effective Theory in the Bulkfor Transport in a Topological Phase, Phys. Rev. B 91, 125303 (2015).

[36] J. M. Link, D. E. Sheehy, B. N. Narozhny, and J. Schmalian, Elastic Response of the Electron Fluid in Intrinsic Graphene: The Collisionless Regime, Phys. Rev. B 98, 195103 (2018).

[37] B. Bradlyn, M. Goldstein, and N. Read, Kubo Formulas for Viscosity: Hall Viscosity, Ward Identities, and the Relation with Conductivity, Phys. Rev. B 86, 245309 (2012).

[38] F. J. Belinfante, On the Current and the Density of the Electric Charge, the Energy, the Linear Momentum and the Angular Momentum of Arbitrary Fields, Physica 7, 449 (1940).

[39] M. Nakahara, Geometry, Topology and Physics (CRC Press, New York, 2003).

[40] L. Rosenfeld, Sur le Tenseur d'Impulsion-Énergie, Mémoires Acad. Roy. de Belgique 18, 1 (1940).

[41] A. Abanov and A. Gromov, Electromagnetic and Gravitational Responses of Two-Dimensional Noninteracting Electrons in a Background Magnetic Field, Phys. Rev. B 90, 014435 (2014).

[42] A. Gromov and A. Abanov, Density-Curvature Response and Gravitational Anomaly, Phys. Rev. Lett. 113, 266802 (2014).

[43] H. J. Lipkin, Lie Groups for Pedestrians (Courier Corporation, New York, 2002).

[44] J. Kogut and L. Susskind, Hamiltonian Formulation of Wilson's Lattice Gauge Theories, Phys. Rev. D 11, 395 (1975).

[45] A. L. Fetter and J. D. Walecka, Quantum Theory of ManyParticle Systems (Courier Corporation, Mineola, NY, 2012).

[46] N. W. Ashcroft and N. D. Mermin, Solid State Physics (Holt, Rinehart and Winston, New York, 1976), Vol. 403 (2005).

[47] J. H. Irving and J. G. Kirkwood, The Statistical Mechanical Theory of Transport Processes. IV. The Equations of Hydrodynamics, J. Chem. Phys. 18, 817 (1950).

[48] For a model with a symmetric stress tensor and no spin, our stress tensor matches Ref. [30].

[49] X. Yu and A. S. Bradley, Emergent Non-Eulerian Hydrodynamics of Quantum Vortices in Two Dimensions, Phys. Rev. Lett. 119, 185301 (2017).

[50] F. D. M. Haldane, Hall Viscosity and Intrinsic Metric of Incompressible Fractional Hall Fluids, arXiv:0906.1854.

[51] F. D. M. Haldane, Geometrical Description of the Fractional Quantum Hall Effect, Phys. Rev. Lett. 107, 116801 (2011).
[52] M. I. Aroyo, J. M. Perez-Mato, D. Orobengoa, E. Tasci, G. de la Flor, and A. Kirov, Crystallography Online: Bilbao Crystallographic Server, Bulg Chem Commun 43, 183 (2011).

[53] M. I. Aroyo, J. M. Perez-Mato, C. Capillas, E. Kroumova, S. Ivantchev, G. Madariaga, A. Kirov, and H. Wondratschek, Bilbao Crystallographic Server I: Databases and Crystallographic Computing Programs, Z. Krist. 221, 15 (2006).

[54] M. I. Aroyo, A. Kirov, C. Capillas, J. M. Perez-Mato, and H. Wondratschek, Bilbao Crystallographic Server II: Representations of Crystallographic Point Groups and Space Groups, Acta Crystallogr. Sect. A 62, 115 (2006).

[55] O. Golan, C. Hoyos, and S. Moroz, Boundary Central Charge from Bulk Odd Viscosity: Chiral Superfluids, Phys. Rev. B 100, 104512 (2019).

[56] D. Forster, Hydrodynamic Fluctuations, Broken Symmetry, and Correlation Functions, in Frontiers in Physics (WA Benjamin, Reading, MA, 1975), Vol. 47, p. 343.

[57] C. Scheibner, A. Souslov, D. Banerjee, P. Surowka, W. T. M. Irvine, and V. Vitelli, Odd Elasticity, arXiv:1902.07760.

[58] J. E. Avron, R. Seiler, and L. G. Yaffe, Adiabatic Theorems and Applications to the Quantum Hall Effect, Commun. Math. Phys. 110, 33 (1987).

[59] T. L. Hughes, R. G. Leigh, and E. Fradkin, Torsional Response and Dissipationless Viscosity in Topological Insulators, Phys. Rev. Lett. 107, 075502 (2011).

[60] N. Read and D. Green, Paired States of Fermions in Two Dimensions with Breaking of Parity and Time-Reversal Symmetries and the Fractional Quantum Hall Effect, Phys. Rev. B 61, 10267 (2000).

[61] J. Xia, J. P. Eisenstein, L. N. Pfeiffer, and K. W. West, Evidence for a Fractionally Quantized Hall State with Anisotropic Longitudinal Transport, Nat. Phys. 7, 845 (2011).

[62] S. Ganeshan and A. G. Abanov, Odd Viscosity in TwoDimensional Incompressible Fluids, Phys. Rev. Fluids 2, 094101 (2017).

[63] A. Abanov, T. Can, and S. Ganeshan, Odd Surface Waves in Two-Dimensional Incompressible Fluids, SciPost Phys. 5, 010 (2018).

[64] A. G. Abanov and G. M. Monteiro, Free-Surface Variational Principle for an Incompressible Fluid with Odd Viscosity, Phys. Rev. Lett. 122, 154501 (2019).

[65] A. G. Abanov, T. Can, S. Ganeshan, and G. M. Monteiro, Hydrodynamics of Two-Dimensional Compressible Fluid with Broken Parity: Variational Principle and Free Surface Dynamics in the Absence of Dissipation, arXiv:1907.11196.

[66] D. Banerjee, A. Souslov, A. G. Abanov, and V. Vitelli, Odd Viscosity in Chiral Active Fluids, Nat. Commun. 8, 1573 (2017).

[67] J. G. E. Harris, D. D. Awschalom, K. D. Maranowski, and A. C. Gossard, Magnetization and Dissipation Measurements in the Quantum Hall Regime Using an Integrated Micromechanical Magnetometer, J. Appl. Phys. 87, 5102 (2000).

[68] S. Mumford, T. Paul, S. H. Lee, A. Yacoby, and A. Kapitulnik, A Cantilever Torque Magnetometry Method for the Measurement of Hall Conductivity of Highly Resistive Samples, arXiv:1908.10857. 
[69] S. A. J. Wiegers, M. Specht, L. P. Lévy, M. Y. Simmons, D. A. Ritchie, A. Cavanna, B. Etienne, G. Martinez, and P. Wyder, Magnetization and Energy Gaps of a High-Mobility $2 D$ Electron Gas in the Quantum Limit, Phys. Rev. Lett. 79, 3238 (1997).

[70] X. Wang, A. M. Jones, K. L. Seyler, V. Tran, Y. Jia, H. Zhao, H. Wang, L. Yang, X. Xu, and F. Xia, Highly Anisotropic and Robust Excitons in Monolayer Black Phosphorus, Nat. Nanotechnol. 10, 517 (2015).

[71] L. Li, F. Yang, G. J. Ye, Z. Zhang, Z. Zhu, W. Lou, X. Zhou, L. Li, K. Watanabe, T. Taniguchi et al., Quantum Hall Effect in Black Phosphorus TwoDimensional Electron System, Nat. Nanotechnol. 11, 593 (2016).

[72] P. J. W. Moll, P. Kushwaha, N. Nandi, B. Schmidt, and A. P. Mackenzie, Evidence for Hydrodynamic Electron Flow in $\mathrm{PdCoO}_{2}$, Science 351, 1061 (2016).

[73] E. M. Spanton, A. A. Zibrov, H. Zhou, T. Taniguchi, K. Watanabe, M. P. Zaletel, and A. F. Young, Observation of Fractional Chern Insulators in a van der Waals Heterostructure, Science 360, 62 (2018).

[74] A. A. Zibrov, C. Kometter, H. Zhou, E. M. Spanton, T. Taniguchi, K. Watanabe, M. P. Zaletel, and A. F. Young, Tunable Interacting Composite Fermion Phases in a HalfFilled Bilayer-Graphene Landau Level, Nature (London) 549, 360 (2017).
[75] M. Serlin, C. L. Tschirhart, H. Polshyn, Y. Zhang, J. Zhu, K. Watanabe, T. Taniguchi, L. Balents, and A. F. Young, Intrinsic Quantized Anomalous Hall Effect in a Moiré Heterostructure, Science 367, 900 (2020).

[76] D. A. Bandurin, I. Torre, R. K. Kumar, M. B. Shalom, A. Tomadin, A. Principi, G. H. Auton, E. Khestanova, K. S. Novoselov, I. V. Grigorieva et al., Negative Local Resistance Caused by Viscous Electron Backflow in Graphene, Science 351, 1055 (2016).

[77] F. A. An, E. J. Meier, and B. Gadway, Direct Observation of Chiral Currents and Magnetic Reflection in Atomic Flux Lattices, Sci. Adv. 3, e1602685 (2017).

[78] N. R. Cooper, J. Dalibard, and I. B. Spielman, Topological Bands for Ultracold Atoms, Rev. Mod. Phys. 91, 015005 (2019).

[79] K. Landsteiner, Y. Liu, and Y.-W. Sun, Odd Viscosity in the Quantum Critical Region of a Holographic Weyl Semimetal, Phys. Rev. Lett. 117, 081604 (2016).

[80] V. Arjona and M. A. H. Vozmediano, Rotational Strain in Weyl Semimetals: A Continuum Approach, Phys. Rev. B 97, 201404(R) (2018).

[81] C. Copetti and K. Landsteiner, Anomalous Hall Viscosity at the Weyl-Semimetal-Insulator Transition, Phys. Rev. B 99, 195146 (2019).

[82] P. Becher and H. Joos, The Dirac-Kähler Equation and Fermions on the Lattice, Z. Phys. C 15, 343 (1982). 